\title{
Dala: A Simple Capability-Based Dynamic Language Design For Data Race-Freedom
}

\author{
Kiko Fernandez-Reyes \\ Uppsala University \\ Sweden \\ kiko.fernandez@it.uu.se \\ Erin Greenwood-Thessman \\ Victoria University of Wellington \\ New Zealand \\ erin.greenwood-thessman@ecs.vuw.ac.nz
}

\author{
James Noble \\ Victoria University of Wellington \\ New Zealand \\ kjx@ecs.vuw.ac.nz \\ Michael Homer \\ Victoria University of Wellington \\ New Zealand \\ michael.homer@vuw.ac.nz
}

\author{
Isaac Oscar Gariano \\ Victoria University of Wellington \\ New Zealand \\ isaac@ecs.vuw.ac.nz \\ Tobias Wrigstad \\ Uppsala University \\ Sweden \\ tobias.wrigstad@it.uu.se
}

\begin{abstract}
Dynamic languages like Erlang, Clojure, JavaScript, and E adopted data-race freedom by design. To enforce data-race freedom, these languages either deep copy objects during actor (thread) communication or proxy back to their owning thread. We present Dala, a simple programming model that ensures data-race freedom while supporting efficient inter-thread communication. Dala is a dynamic, concurrent, capability-based language that relies on three core capabilities: immutable values can be shared freely; isolated mutable objects can be transferred between threads but not aliased; local objects can be aliased within their owning thread but not dereferenced by other threads. Objects with capabilities can co-exist with unsafe objects, that are unchecked and may suffer data races, without compromising the safety of safe objects. We present a formal model of Dala, prove data racefreedom and state and prove a dynamic gradual guarantee. These theorems guarantee data race-freedom when using safe capabilities and show that the addition of capabilities is semantics preserving modulo permission and cast errors.
\end{abstract}

Keywords: concurrency, capability, permission, isolation, immutability

\section{ACM Reference Format:}

Kiko Fernandez-Reyes, James Noble, Isaac Oscar Gariano, Erin Greenwood-Thessman, Michael Homer, and Tobias Wrigstad. 2021. Dala: A Simple Capability-Based Dynamic Language Design For

Permission to make digital or hard copies of all or part of this work for personal or classroom use is granted without fee provided that copies are not made or distributed for profit or commercial advantage and that copies bear this notice and the full citation on the first page. Copyrights for components of this work owned by others than ACM must be honored. Abstracting with credit is permitted. To copy otherwise, or republish, to post on servers or to redistribute to lists, requires prior specific permission and/or a fee. Request permissions from permissions@acm.org.

Conference'17, fuly 2017, Washington, DC, USA

() 2021 Association for Computing Machinery.

ACM ISBN 978-x-xxxx-xxxx-x/YY/MM...\$15.00

https://doi.org/10.1145/nnnnnnn.nnnnnnn
Data Race-Freedom. In Proceedings of ACM Conference (Conference'17). ACM, New York, NY, USA, 39 pages. https://doi.org/10.1145/ nnnnnnn.nnnnnnn

\section{Introduction}

Most mainstream object-oriented languages do not rule out data races - read-write or write-write accesses to a variable by different threads without any interleaving synchronisation. This makes it hard to reason about the correctness, or in programming languages like $\mathrm{C}$ and $\mathrm{C}++$ even the meaning of programs. Static languages such as Java or Go have higher level constructs to control concurrency but, ultimately, nothing strictly prevents data races from happening. Dynamic languages such as Ruby or Python repeat the same story: nothing prevents data races, not even a global interpreter lock.

Many object-oriented languages added concurrency constructs as an afterthought, and objects may suffer data races. Data race free languages have implicit concurrent properties as part of the "object model" and guarantee data racefreedom. Examples of data race free languages are: $\mathrm{E}$ which uses object capabilities and far references to forbid access to (global) and un-owned resources [56], capability- or ownershipbased languages such as Pony $[19,20]$ or Rust [55], or languages without mutable state, e.g., Erlang [2].

Freedom from data races simplifies avoidance of race conditions, which happen when behaviour is controlled by factors outside of the program's control, such as the scheduling of two threads. Data race free languages thus have a leg up on "racy" languages in this respect, but data race freedom always comes at a cost: languages either deliver "efficient concurrency" or "simple concurrency" but not both.

Table 1 shows the features of eight data race free languages. (Dala is our proposal, and we discuss its features later in the paper.) The first four languages are statically typed. To maintain data race-freedom, these introduce new concepts which permeate a system to: ownership, capabilities, capability composition, capability subtyping, capability promotion and recovery, and viewpoint adaption [13, 18, 20, 31, 55] (though 
Table 1. Summary of features of capability-based static languages

\begin{tabular}{|c|c|c|c|c|c|c|c|c|c|}
\hline Complexity $\backslash$ Languages & \multicolumn{4}{|c|}{ Pony Rust Encore Reflmm } & \multicolumn{4}{|c|}{ E Newspeak AmbientTalk Erlang } & \multirow{2}{*}{$\frac{\text { Dala }}{3}$} \\
\hline Capabilities & 6 & 5 & $7+$ & 4 & $\checkmark$ & $\checkmark$ & $\checkmark$ & $x$ & \\
\hline Capability Subtyping & $\checkmark$ & $x$ & $\checkmark$ & $\checkmark$ & $x$ & $x$ & $x$ & $x$ & $x$ \\
\hline Promotion, Recovery, Borrowing & $\checkmark$ & $\checkmark$ & $\checkmark$ & $\checkmark$ & $x$ & $x$ & $x$ & $x$ & $x$ \\
\hline Compositional Capabilities & $\checkmark$ & $\checkmark$ & $\checkmark$ & $x$ & $x$ & $x$ & $x$ & $x$ & $x$ \\
\hline Deep copying & $x$ & $x$ & $x$ & $x$ & $\checkmark$ & $\checkmark$ & $\checkmark$ & $\checkmark$ & $x$ \\
\hline Far References & $x$ & $x$ & $x$ & $x$ & $\checkmark$ & $\checkmark$ & $\checkmark$ & $x$ & $x$ \\
\hline Data-Race Freedom & $\checkmark$ & $\checkmark$ & $\checkmark$ & $\checkmark$ & $\checkmark$ & $\checkmark$ & $\checkmark$ & $\checkmark$ & $\checkmark$ \\
\hline
\end{tabular}

not necessarily all at once). This may have lead to a steep learning curve [51]. In return, these languages deliver efficient concurrency: they allow large object graphs to be shared or passed around safely by pointer, or allow multiple threads to access different places of a single data structure at once.

The next four languages are dynamically typed. These maintain data race-freedom implicitly and requires the programmer to do little or nothing: objects are either copied between "threads" [39] (which, while simplifying garbage collection, may be expensive [60] and loses object identity), or proxied back to the "thread" that owns them [12, 27, 57, 71], which adds latency and makes performance hard to reason about unless it is clear what operations are asynchronous. ${ }^{1}$

The goal of this work is to deliver a design that provides both simplicity and performance, and that may work both in dynamically and statically typed programming languages. To this end we present Dala, ${ }^{2}$ a capability-based and dynamic approach to data race-freedom without mandating deep copying and without the typical complexity of capability systems. The Dala model allows mixing objects which are guaranteed to be safe from data-races with objects that are not and thereby supports the gradual migration of programs to using only "safe objects," by converting unsafe objects to safe objects one at a time. Dala uses three object capabilities to maintain data race-freedom: immutable values that can be shared freely; isolated mutable objects that cannot be aliased but can be transferred between threads, and thread-local objects that can be aliased across threads but only dereferenced by the thread that created them. In this paper, we study our capabilities in a very simple setting: an untyped object-based language, leaving optional static typing for future work.

To support the design of Dala we contribute Dalarna, a formal model of the core of Dala. We use this model to prove that objects with capabilities cannot be subject to data races, nor can they observe a data race except via explicitly unsafe parameters passed to their methods. We also show that Dala supports a form of gradual guarantee $[69,70]$ : the addition

\footnotetext{
${ }^{1} \mathrm{E}$, Erlang, and AmbientTalk are also distributed languages which motivates their copying and proxied reference approaches.

${ }^{2} \mathrm{~A}$ "Dala Horse" is a small carved wooden toy horse - a handmade little pony $[20,32,64])$.
}

of capabilities preserves the dynamic semantics, modulo runtime errors that check that the program behaves according to the programmer's explicitly stated intentions, e.g., disallowing a write to an immutable object. The Dala model can be embedded into a wide range of garbage-collected objectoriented languages, such as Java, TypeScript, Ruby, OCAML, Swift, Scala, Go etc. To demonstrate that Dala has the potential to be practicable, for both statically and dynamically typed programs, we have a proof-of-concept implementation, Daddala, which embeds Dala in Grace [3, 41, 59], built on top of Moth VM [63].

\section{Contributions and Outline.}

1. We overview three inherent problems in race unsafe and safe programming languages, and discuss the current approaches in ownership- and capability-based systems (§2).

2. We introduce the Dala capabilities that allow safe interaction between programs containing data races from parts that should remain data race-free $(\S 3)$.

3. We show how Dala tackles the three inherent problems in $\S 2(\S 4)$.

4. We provide a formal description of Dala and its core properties ( $\$ \S 5$ and 5.3).

5. We provide Daddala, a proof-of-concept implementation that (anecdotally) shows the relative ease of embedding Dala in an existing system $(\S 6)$.

$\S 7$ places Dala in the context of related work, and $§ 9$ concludes.

\section{Background: Perils of Concurrent Programming}

To set the scene for this paper we discuss common problems in race unsafe (e.g., Java) and safe concurrent languages (e.g., Rust). First we discuss balancing complexity and performance (\$2.1); then how tying safety to particular concurrency abstractions leads to a one-size-fits-all model which leads to problems with compositionality of concurrent abstractions (§2.2); last we discuss the problem of providing escape hatches to permit behaviour that is not supported by the programming language (§2.3). 


\subsection{Balancing Safety, Complexity and Performance}

Safe languages have (implicit) concurrency mechanisms to prevent data races. From Table 1, we argue that the constructs or systems fall into three categories: complex and efficient, simple and inefficient, and complex and inefficient.

Complex and Efficient Pony, Rust, Encore as well as Gordon's work on reference immutability are race-safe by controlling access to shared data, rather than banning its existence $[8,19,20,35,55]$. This is achieved by providing concepts that are not common to every day developers, such as capability and ownership type systems. These allow both efficient and data race-safe sharing and transfer of ownership with reference semantics. This allows e.g., passing a large data structure across actors by reference, which subsequently allows fast synchronous access by the receiving actor. Implementing a concurrent hashmap in these systems requires up-front thinking about how keys and values may be accessed across different threads, and mapping the intended semantics onto the types/capabilities that the languages provide. For example, in Pony, a concurrent hashmap will be an actor; to allow multiple threads to know the existence of the same keys, the keys must be immutable; etc.

The cost of safe efficient data sharing is complexity: capability type systems introduce complex semantics such as capability promotion, capability subtyping, capability recoverability (e.g., getting back a linear reference after it was shared), compositional capability reasoning (i.e., combining capabilities to produce new capabilities), and viewpoint adaption (i.e., how to view an object from another object's perspective, e.g., Pony uses viewpoint adaption to write parametric polymorphism [53]). Understanding these concepts is key to write code that is efficient and free from data races.

Some form of unique/affine/linear reference is often the cornerstone of many of the static capability systems. Such references are extremely powerful: they provide reasoning power, they can often be converted into other capabilities (e.g., to create cyclic immutable data structures) or to transfer ownership of objects across threads. However, polymorphic behaviour is typically a source of great pain for these systems. For example, consider a simple hashmap - concurrent or not. If values can be unique, a lookup must remove the value out of the data structure to preserve uniqueness (and the associated entry to reflect this in the hashmap). If values are not unique, this behaviour is counter-productive. Behaviours like this force duplication of code.

Simple and Inefficient ASP [11], ProActive [10], Erlang [2], E [56], AmbientTalk [23, 28], Newspeak [6, 7], functional objects in ABS [42], among others, avoid data races by deep copying objects in messages (for some languages modulo far references, see Complex and Inefficient). This approach is relatively simple and the price for data race-freedom is copying overhead paid on every message send.
In addition to consuming $\mathrm{CPU}$ cycles and increasing the memory pressure, deep copying also loses object identity, and requires traversal of the objects transferred, a $O$ (\#objects) operation, possibly requiring auxilliary data structures (adding overhead) to preserve internal aliasing of the copied structure.

Notably, Erlang requires two such traversals: the first calculates the size of all objects to enlarge the receiving process' heap and the second copies them across. Erlang's deep copying is key to keeping process' heaps disjoint, which simplifies concurrent garbage collection and reduce overall system latency. In languages that compile to Java, like ABS, ASP, ProActive, etc., the run-time is unable to see or leverage such isolation.

A concurrent hashmap in Erlang has no choice: its keys and values must be immutable, and are therefore safe to share across multiple processes. The internal hashmap data structures must be immutable too, which might be less efficient. $^{3}$

Complex and Inefficient To support safe sharing without losing object identity, E [56], AmbientTalk [23, 28], and Newspeak [6, 7] support far references. A far reference is a proxy that allows an object owned by an actor/process to directly reference another's innards, but all interaction with the proxy is lifted into an asynchronous message and sent back to the owner, to be executed there. Thus, despite the fact that many actors can point directly to an object, only its owning process will ever read or write the object. Thus, with proxies, there is a cost per access which can be expensive [60].

In $\mathrm{E}$ and AmbientTalk, proxied (far) references must be operated on asynchronously and only non-proxied (local) references allow synchronous access. Code that needs to be "proxy-agnostic" must use asynchronous access. In E and AmbientTalk, promises are also implicit proxied references. ${ }^{4}$ This means that asynchronous sends $(x \leftarrow)$ may be delayed indefinitely if the promise is never fulfilled, meaning the $x$ 's value is never materialised so there is no recipient of the message.

Implementing a concurrent hashmap in AmbientTalk (E and Newspeak are similar) does not need any capability annotations to track how values stored in the map may be shared across threads. Promises remove the need for callbacks, but indirections make the implementation more complex or convoluted. As a blocking synchronisation on the result of a promise is not possible, we must use the promise chaining operator to access the (possible) value in the promise.

While syntactically simple, the inability to access resources directly makes reasoning about performance hard. The main

\footnotetext{
${ }^{3}$ Erlang Term Storage provides a way to escape this design of Erlang, but at a cost of dropping to a much lower-level of programming and manual memory management.

${ }^{4} \mathrm{E}$ calls them promises, AmbientTalk calls them futures. We will refer to them as promises [54].
} 
implication of not allowing direct access is that we neither know if far references are ever going to be fulfilled nor if the promise chaining combinator (on far references) makes the owning actor (of the promise) the bottleneck of the system.

\subsection{Safe One-Size-Fits-All Concurrency}

In race unsafe languages, abstractions are broken by the addition of concurrency constructs. For example, inconsistencies in an object's internal state during a method's execution, which are hidden in a purely sequential system, may be observed if the object can be accessed concurrently. This problem of object-oriented languages and their "unsafe" concurrency features was first studied by M. Papathomas [61]. Yet, there are plenty of abstractions and programming models that guarantee data-race freedom, such as implementations of the actor model [14, 25, 76]. In languages like $A k k a$ [76], the model is data race-free as long as all code in a system adheres to a set of guidelines [1].

These guidelines tie concurrency safety properties to their concurrency model, suffering from "one-size-fits-all" problem. For example, Akka can only guarantee data race-freedom when the program (follows the guidelines and) stays within the actor model. Spawning threads within an actor can easily break its concurrency safety properties, e.g., data racefreedom.

Thus, to implement a concurrent hashmap, an Akka program might simply wrap the standard Java hashmap in an actor. As long as the hashmap itself is never leaked from the actor, and the actor does not create additional threads, all updates the hashmap will be sequential and therefore free from data races. Whether the keys and values are safe from data races is beyond the control of the hashmap, and is ultimately up to the diligence of the programmers (e.g., to maintain uniqueness or immutability).

In E, AmbientTalk, and Newspeak, (as well as many languages that did not fit in Table 1 such as ABS, Pony, Proactive and others), the mechanisms that guarantee data racefreedom are inherently linked to the languages' chosen concurrency models. In this case, the near and far references span (affect) other concurrency models. Using the example from before, global objects can be safely protected by locks but this cannot be easily accommodated ${ }^{5}$ and the run-time may throw an exception when multiple threads have access to a shared object [24].

\subsection{Safety May Beget Unsafety}

In safe languages based on capabilities or ownership types (e.g., $[8,19,20,55])$ it is sometimes necessary to side-step the type checker, to write low-level code that interacts with hardware, or when the type system is not "clever enough" to allow a correct behaviour. We exemplify these cases in turn.

\footnotetext{
${ }^{5}$ This can be partially mitigated by adding futures, which imposes again other concurrent semantics to maintain the safety properties [24]
}

Hardware Meets Software. Graphic cards constantly read from video memory and developers can write directly on the frame buffer that points to the video memory to update the image in the next refreshing cycle. Implicitly, this means that there is a data race between the graphics card and the main thread; such racing behaviour may show flickering of the image on screen. A double buffering technique removes this flickering, using an on-screen buffer that the graphics card reads, and one off-screen where developers write the next scene. A swap operation swaps the buffer pointers from offto on-screen. This common - and racy - approach prevents the flickering.

Trust Me, I Know What I Am Doing. As all statically typed languages, capability- and ownership-based programming languages are engaged in a balancing act of expressivity and complexity. Simplifications made to the systems to reduce the programmer's overhead will invariably lead to exclusion of valid programs, simply because the system is not powerful enough to express its behaviour within its model.

We can illustrate this using the concurrent hashmap example in the context of the Encore programming language. Encore suffers from the problem mentioned in $\S 2.1$ where a hashmap must choose between supporting unique references (and therefore always moving values in an out), or not (allowing them to be in the hashmap at the same time as they are referenced elsewhere). This can be solved in Encore by dropping to the underlying language to which Encore compiles where no such checks are made, and implement, for example, parallel put and get methods that accepts linear values even though this is unsound in the type system, and transfers them (hopefully) correctly. Other languages (e.g., Rust) may have an unsafe block, or provide reflective constructs that are unsafe from a capability perspective. Naturally, all such code gives rise to technical debt.

In order to circumvent shortcomings of the capability systems, programmers may resort to escape hatches that void the guarantees of data-race freedom. If data races happen inside an escape hatch, the behaviour is undefined [21, 45]. In Pony (and Encore), if an unsafe block introduces a data race the run-time (garbage collector) might eventually crash [21]. There is no safe interoperability between unsafe and safe code!

\subsection{Summary}

The data race-freedom guarantee of safe languages comes at a cost of complexity or inefficiency, or both. Furthermore, most or all languages' safety is tied to a specific concurrency model, and may not compose with others. Finally, data races are sometimes desired, or a systems' notion of safe is too safe to express correct code. Escape hatches overcome these problems, but at a cost of losing data race-freedom.

In the next section, we describe our simple model that is efficient, concurrency-model agnostic, and provides safe interoperability between unsafe and safe code. 


\section{An Overview of The Dala Model}

Objects in Dala are associated with capabilities that describe how they can interact with other objects. The association happens at creation time and is fixed for life. We refer to immutable, isolated, and thread local as the "safe capabilities". Programs which only operate on a safe heap are guaranteed to be data-race free. When it is not important to distinguish between an object and its capability, we will say e.g., "a local object" to mean an object with a local capability or a "safe object" etc.

The following code creates an immutable object with a field $f$ with value $v$ and a unary method $m$ with body $t$ : object $\{$ use imm; def $f=v$; method $m(x)\{t\}$ \}. Fig. 1 shows the interaction of capabilities: arrows show all legal references from an object with one capability to another, modulo reflexivity.

The Dala capabilities form a hierarchy; imm $<$ iso $<$ local $<$ unsafe. Objects can only refer to other objects with the same or lesser capabilities: an immutable object can only refer to other immutable objects; an isolate object can refer to other isos or imms, and a local object can refer to imms, isos, and other local objects. The model treats objects outside Dala as having a fourth unrestricted unsafe capability for uniformity. Using unsafe objects in a Dala program makes it susceptible to data races. Because it is the top capability, objects in the safe heap (imms, isos, locals) cannot refer to objects in the unsafe heap, while unsafe objects can refer to anything (see Fig. 2). The implication of this model is that once an object is safe, its entire reachable object graph is safe as well. Thus: data races can only occur in unsafe objects. By including unsafe objects in the model, we can describe the semantics of a program partially annotated with Dala capabilities.

Avoiding data-races by ensuring that two threads do not concurrently execute a code block that accesses the field $f$ of some object $o$ at the same time focuses on code. If there is another place in the program that also accesses o's $f$ field which could be run at the same time, a data race could still happen. Making the object safe means that all code that interacts with $o$ must follow the rules that make $o$ safe from data races. If $o$ is local, it cannot be shared across threads, so all accesses to its $f$ field will come from the same thread. If $o$ is immutable, all accesses to $f$ will be read accesses which are benign. If $o$ is isolated, two accesses to its $f$ field by different threads require an explicit transfer from the first thread to the second thread (possibly via additional "stop-overs").

Dala capabilities are self-protecting in the sense that safety stems from a capability's own internal restrictions, not from restrictions elsewhere in the system. Writing to a field of an imm throws an error; so does aliasing an iso ${ }^{6}$, or accessing a

\footnotetext{
${ }^{6}$ This property can be implemented by ensuring that all code external to an iso accesses it via a proxy object with movement semantics, e.g., by overloading the $=$ operator. A simple implementation is possibly using
}

local object from outside of the thread it is local to. Imagine that objects' fields are private, and that field accesses implicitly go through a getter/setter indirection - in this case all the checks necessary for an object to maintain its invariants are in its own internal code. This is an important part of the design and key to adding unsafe capabilities in cases where these cannot be expected to be cooperative in avoiding data races. Fig. 3 shows how capabilities restrict certain effects in the system. Due to the absence of static types, these restrictions are enforced at run-time, meaning the cost of data-race freedom is (roughly) per access.

Dala's isolated objects are the key to efficient transfer of mutable state between different threads. (Immutable objects can be shared directly without causing problems, and thread local objects are permanently confined within their owning thread.) Isolates have only one unique incoming reference, and some extra care must be taken to preserve this uniqueness. Dala incorporates an explicit consume operation that destructively reads [40] the contents of a variable, and prevents those contents from being used again, similar to C++'s "move semantics". The contents of any mutable variable may be consumed but variables containing isolates must be consumed-otherwise the attempt to read the isolate will fail. Experiences by Gordon et al. [35] suggest explicit consume is to be favoured over implicit

The consume operator succeeds on all variables except the special self variable. This makes an isolated self variable effectively borrowed [4], meaning its value is tied to the current stack frame. Because the value of self cannot escape, expressions such as $x \cdot m(y)$ when $x$ is an isolated object do not need to consume $x$ and are guaranteed not to introduce any alias to $x$, other than through self on subsequent stack frames. With this borrowing-like behaviour, it is possible to traverse isolated structures without consuming them (a wellknown problem when dealing with unique or linear values), but notably only using internal methods: $x . m$ is allowed to borrow whereas $m(x)$ is not. Since overcoming this limitation is well-known, we refrain from discussing this any further.

Dala guarantees that data races can only happen in places with unsafe objects, never in objects with safe capabilities (Theorem 5.5: Data Race Freedom). Developers can easily add capabilities to migrate from a "racy" program to a data race-free program with the certainty that this migration is semantics preserving, modulo permission and cast errors (Theorem 5.6: Dynamic Gradual Guarantee). These two properties are key in Dala and we will formally state them in $\S 5.3$.

\subsection{Simple Case Study: A Concurrent Hash Map}

To illustrate the simplicity of the Dala capabilities, consider the implementation of a simple concurrent hashmap in Fig. 4.

linear proxies and allows unsafe objects to race on iso stack variables (as they already can on fields in Dala). 


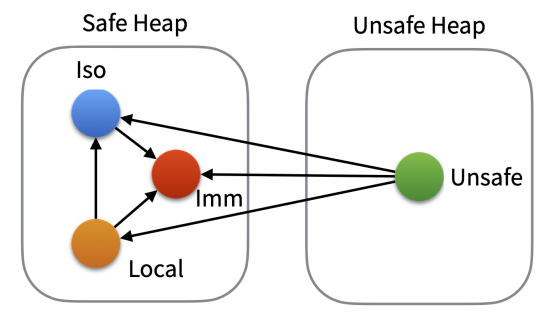

Figure 1. Dala Heap.

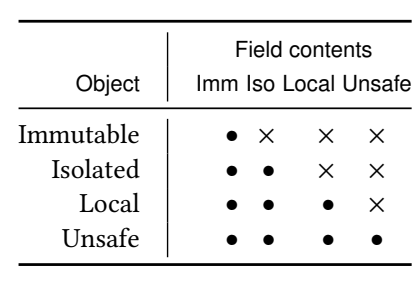

Figure 2. Structural restrictions.

\begin{tabular}{r|cccc}
\hline & \multicolumn{4}{|c}{ Effects } \\
Object & Read Write Alias Transfer \\
\hline Immutable & $\bullet$ & $\times$ & $\bullet$ & $\bullet$ \\
Isolated & $\bullet$ & $\bullet$ & $\times$ & $\bullet$ \\
Local & $\bullet$ & $\bullet$ & $\bullet$ & $\times$ \\
Unsafe & $\bullet$ & $\bullet$ & $\bullet$ & $\bullet$ \\
\hline
\end{tabular}

Figure 3. Capabilities and effects.
Assume that the hashmap is created inside a lightweight process, eventually calling the tail-recursive run() method with the channel msgs. The run() method reacts to input sent on the channel. It dispatches on the op field of messages received, and additionally expects the fields key and val to be present depending on operation.

A hashmap has five moving pieces: the hashmap object itself, the array of buckets, the entries in the buckets, and the keys and values of the entries. We examine the possible capabilities of these in turn to ensure thread-safety of the hashmap implementation.

For keys and values, there are two possibilities: iso and imm. In the former case, the keys and values can be transferred between the hashmap and its clients. In the latter case, they can be shared but also never change. It makes sense for keys to be immutable so that any client thread can know of their existence and ask for their associated value. We capture this in the code on Line 7 by calling the built-in freeze() operator that is the identity function on immutables, or creates an immutable copy otherwise (like in Ruby).

For values, iso and imm make sense under different circumstances. A sensible hashmap implementation should store only a single reference to its values, so the same code base should be reusable in both scenarios. The code in Fig. 4 support values which are both iso and imm. The key lines are $9,11,21,34$, and 40 which always move a value. Our field assignment makes use of "swap semantics" where the old value of the field is returned on an update. As a result, a get operation will move the value associated with a key out of the hashmap and remove the corresponding entry (Lines 20-21). (But see $§ 4.3 .1$ !)

From a thread-safety perspective, linked entries that constitute a bucket could be either immutable, iso, or local. Immutable entries would complicate the code when entries are unlinked (Line 20). Iso entries would slightly complicate searching through linked entries while maintaining uniqueness (lines 16-18 and 29-32). Thus, our entries are local (Line 37).

A similar thought process applies to the array of buckets. However, as our entries are local, we have no choice but to make the array local as well. For simplicity, the implementation of the array is elided above but we include Line 48 to explicitly show this choice. (As suggested by lines 15-16 and
27-29, our implementation assumes an empty bucket has a dummy entry for simplicity.)

Consequently, the hashmap itself must be local (Line 2). This ties the hashmap to the thread or lightweight process where it was created which does not seem unreasonable.

3.1.1 Gradual Transition to Dala Capabilities. Notice the bottom-up thinking when assigning the capabilities in the previous section. In a top-down approach, we might have decided to make the hashmap an iso to support its movement across threads or processes. This would have excluded local from the possible capabilities for the buckets array and its entries. When retrofitting existing code to use Dala capabilities, the bottom-up approach is superior to the top-down approach as it will incur the smallest possible change to the program. Assume the code in Fig. 4 was written as now, but without capabilities in mind: including no use declarations on Lines 2, 37 and 48, no consumes on Lines 34 and 40 , and no freeze() on Line 39 , etc. In this case, changing the keys to be immutable is simply adding the freeze() call on Line 39. All other objects in the hashmap can remain as-is. Similar, making values isos only needs the two consume operations, and changing msg. val to msg.val = null on lines 9 and 11.

Because of the structural constraints imposed on objects with a capability, top-down migration is likely to require bigger changes. For example, adding the use local on Line 2 will require adding a capability to the bucket array on Line 47-49. This will propagate to the entries and their keys and values.

A good regression test suite might be useful to help drive adding annotations regardless of the approach.

3.1.2 Constructing and Using the Hashmap. While orthogonal to the Dala capabilities, Fig. 5 illustrates how a program might construct a hashmap (lines 1-8) by spawning a new process with an associated channel that creates the hashmap and connects it to the channel.

When calling the constructor (Line 9), the caller gets a channel that can be used to send messages to the hashmap. It is easy to construct a proxy for a map that captures the channel used to communicate with the map, and uses a dedicated channel to get the reply (lines 11-24). The current map_proxy creates a new channel per interaction with the concurrent hashmap. This allows it to be immutable. If the 


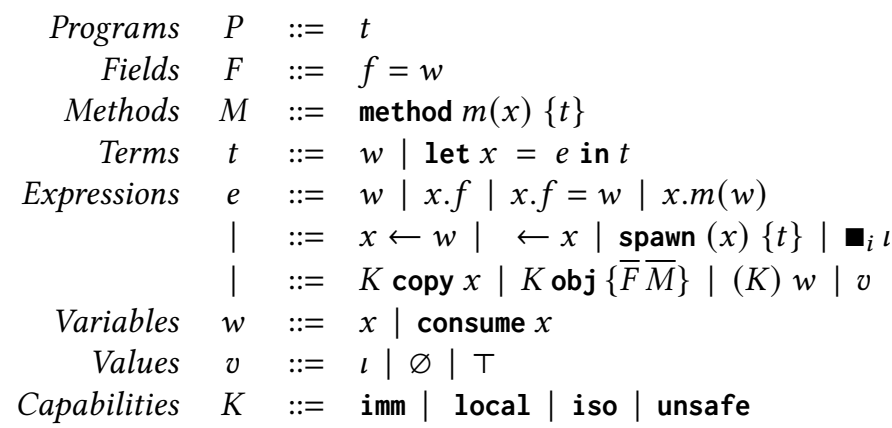

Figure 6. Syntax of Dalarna. $m, f$, and $x$ are meta-variables representing method, fields, and variable names; metavariable $x$ includes self. $\varnothing$ is a run-time value representing an empty channel.

of local capabilities freely. Since local objects cannot contain unsafe objects, any data race due to this weakening is not visible to the local object, which voids the need for unsafe propagation.

4.3.1 Supporting both Immutable and Isolated Values in a Hashmap. Because of the inherent flexibility of dynamic checking, it is simple to add an additional operation that behaves like get, but returns an alias to the value in the hashmap of Fig. 4. This operation would throw an error if used on isos, but work fine on immutable objects. We can even implement it by extending the existing get method:

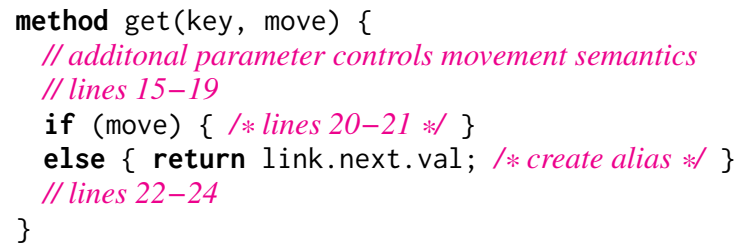

This overcomes the problem pointed out previously forcing developers to choose one particular semantics or duplicate code, and does not compromise soundness.

\section{Formalising the Dala Capability and Concurrency Models}

To formally study the Dala model and clearly state and prove its key properties (Data Race-Freedom and Dynamic Gradual Guarantee), we formalise Dala in a minimal concurrent object-based language which we call Dalarna, which we describe in this section. In Dalarna, objects have the usual fields and methods, and there are no classes and no inheritance. Threads are created by a spawn operation which also sets up a channel for communication. Channels support both reference semantics and value semantics for objects depending on their capability meaning Dalarna is a shared-memory model. However, with the exception of unsafe objects, two threads sharing a common object $o$ cannot implicitly transfer objects between each other by reading and writing fields in $o$. Hence, with the exception of unsafe objects, objects are effectively running in a message passing model, which may use shared memory under the hood for efficiency, without compromising safety.

Not modelling classes or inheritance is a choice driven by the desire to keep the system minimal. We note that dynamically typed languages are less dependent on inheritance, because of the non-need to establish nominal subtyping relations. Our simplifications allow us to focus on the most important aspects of our work. Handling permissions and capabilities in the presence of various forms of inheritance is well-known (e.g., [13, 15, 48, 72, 77]) in a statically typed world including problems that may arise in an untyped setting. Therefore, we do not believe that these simplifications accidentally suppress any fundamental limitations of our approach.

For simplicity, channels themselves cannot be transferred. There is nothing fundamental about this simplification but undoing it requires some design thinking which is not important for the paper at hand, such as, whether or not we allow multiple threads connected to a single channel to race on taking the next message, etc.

Figure 6 shows the syntax of Dalarna. As is common, overbars (e.g., $\bar{f}$ ) indicate possibly empty sequences (e.g., $\left.f_{1}, f_{2}, f_{3}, \ldots\right)$. To simplify the presentation of the calculus programs are in A-normal form [33]: all subexpressions are named except for the consume expression. We further assume that programs use static single-assignment form [65], i.e., the let-in term always introduces a new variable, field reads are assigned to variables before they are bound to other variables, etc, and that self is never aliased. None of these constraints are essential for the soundness of our approach.

A program $(P)$ is a term $(t)$. Terms are variables and letbound expressions (i.e., $x$ and let $x=e$ in $t$ ). An unusual design choice borrowed from [20] is that assignment, $L=R$, binds the left-hand side $L$ to the value of the right-hand side, $R$, and returns the previous value of $L$. (This is not uncommon when dealing with iso fields, and previous work in this area enforce it via an explicit swap operation [37-39]). Expressions are variables $(w)$, destructive reads (consume $x$ ), field reads $(x . f)$, field assignments $(x . f=w)$, method calls $(x . m(w))$, a deep copy operation (K copy $x$ ) that returns a copy of an object graph with capability $K$, an object literal, a casting operation that asserts a capability $((K) v)$, and a spawn operation that creates a new thread. At run-time, the expression syntax also includes values. An object consists of fields $(\overline{f=x})$ and methods $(\bar{M})$ and are instantiated with a given capability $K$. For simplicity, methods have a single argument (e.g., $x$ ) and more can be modelled using an object indirection using an unsafe object.

Spawning a new thread using spawn $(x)\{t\}$ introduces a new channel $x$ both at the spawn-site, and inside the scope of the new thread whose initial term is $t$. The $t$ is closed, 


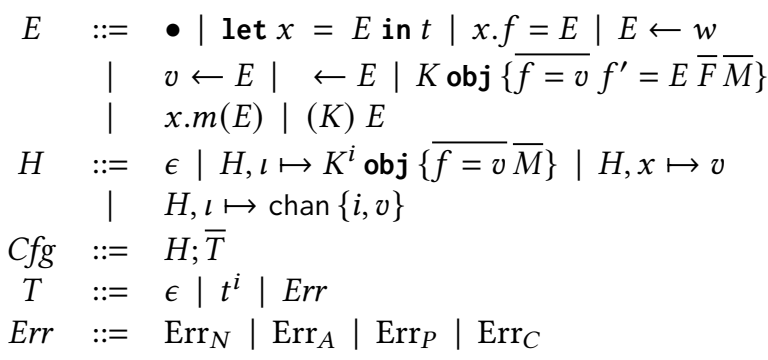

Figure 7. Definitions of evaluation context, store and runtime configurations.

i.e., it cannot access variables declared from an outer scope. Channels are bidirectional unbuffered with blocking semantics on sending and receiving operations. The send operation $x \leftarrow w$ puts $w$ on channel $x$, if necessary blocking until the channel is "free" (i.e., contains $\varnothing$ ). The sender then blocks $\left(\mathbf{\square}_{i} \iota\right)$ until the message $i$ is received by the thread on the other end of the channel $\iota$. The receive operation $\leftarrow y$ is similar to a send and blocks the current thread while the channel is "free". Values are locations ( $l$ ), the "absent value" $T$ used to populate a consumed variable or field, and $\varnothing$, used at run time to indicate that a channel is empty.

For simplicity, we assume that programs do not attempt to consume self, which can be enforced through a simple syntactic check, and that all variable/method-parameter/channel names are distinct and none is called self. This is a common restriction in the literature, and key to avoiding breaking of abstraction [16].

\subsection{Dynamic Semantics}

We formalise the dynamic semantics of Dala as a small-step operational semantics with reduction-based, contextual rules for evaluation within threads (Fig. 7). The evaluation context $E$ contains a hole $\bullet$ that denotes the location of the next reduction step [74]. We write the reduction step relation $H ; \bar{T} \leadsto H^{\prime} ; \overline{T^{\prime}}$ which takes a reduction step from heap $\mathrm{H}$ and a collection of threads in $\bar{T}$, to a new heap H' and a new thread state $\overline{T^{\prime}}$. A store $H$ is either empty $(\epsilon)$, or contains mappings from variables to values, and from locations to objects and channels (Fig. 7). The superscript $i$ in $K^{i}$ represents the object's thread owner and we used it to keep track of ownership of local objects (omitted from the rules when not relevant).

A configuration $C f g$ is a heap $H$ and a collection of concurrently executing threads $\bar{T}$. A thread is either finished $(\epsilon)$, a term $t^{i}$ (where $i$ represents the thread owner id, omitted when not necessary), or a run-time error (Err). There are four kinds of run-time errors: consumption errors $\left(\operatorname{Err}_{A}\right.$, which occur when a program accesses a consumed value); permission errors $\left(\operatorname{Err}_{P}\right.$, which occur when a program violates the structural constraints imposed by its capabilities); cast errors $\left(\operatorname{Err}_{C}\right.$, which occur when a program has a different capability than the one casted to); and normal errors $\left(\operatorname{Err}_{N}\right)$, such as accessing a non-existing field, calling a nonexisting method, etc. The execution of threads is concurrent and non-deterministic. The non-determinism comes from C-Eval and the commutativity equivalence rule.

$$
\begin{aligned}
& \text { (C-EvaL) }
\end{aligned}
$$

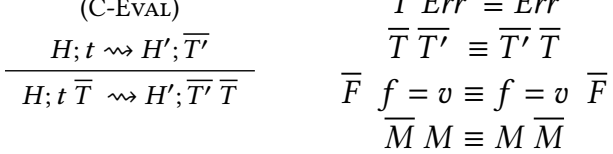

The reduction of a program $t$ begins in an initial configuration with an empty heap $\epsilon ; t$ (Definition B.1, §5.3). In the remainder of this section, we go through the reduction rules, ending with a discussion of the error trapping rules that dynamically trap actions which (might) lead to data races. For capabilities, the following relations hold: unsafe $\leq$ local, local $\leq$ iso, and iso $\leq \mathrm{imm}$. The $\leq$ relation is reflexive and transitive. isImm $(H, \iota)$ (etc. for other capabilities) holds if $H(\iota)=K$ obj $\left\{{ }_{--}\right\}$and $K=\mathbf{i m m}$.

The reduction of the let term updates the heap with a stack variable $x$ pointing to the value $v$ (R-LET). Reading a variable with a non-isolated object reduces to a location (RVAR); reading an isolated object involves moving semantics: moving the contents of a variable using consume reduces to a location, and leaves a $T$ token in the variable which will cause an error if accessed before overwritten (R-ConsumE). Consuming fields is not allowed. Instead, one consumes a field when doing an assignment, e.g., let $x=(y . f=z)$ in ..., places on $x$ the object pointed by $y . f$ and places $z$ in $y . f$. Reading a field $(x . f)$ reduces to the value stored in the field (R-FIELD). Note that to read an isolated object's field one must update the field and place another object in its stead directly reading an isolated field would create a new alias. The helper predicate localOwner(...) checks that if the target object is local, then the current thread is its owner. (This prevents threads to access unowned local objects.)

Updating a field $(x . f)$ with a value $(v)$ reduces to returning the previously held value in the field and updating the field $f$ to point to value $v$. There is a check that prevents mutating immutable objects, $\operatorname{OkRef}(H, K, v)$ ensures that the object $v$ can be placed under an object with capability $K$, and the remaining helper predicates check that if the target object $(x)$ is local, then its owner is the current thread and if the source $(v)$ is local then its owner is the current thread. ${ }^{9}$ (Other reduction rules repeat this local ownership check and for space reasons we omit mentions in the remaining rules.)

\footnotetext{
${ }^{9}$ This design is good for JIT compilation since local objects have guarantees to not point to unowned local objects (Corollary 5.3), thus removing some unnecessary dynamic checks from possible implementations.
} 


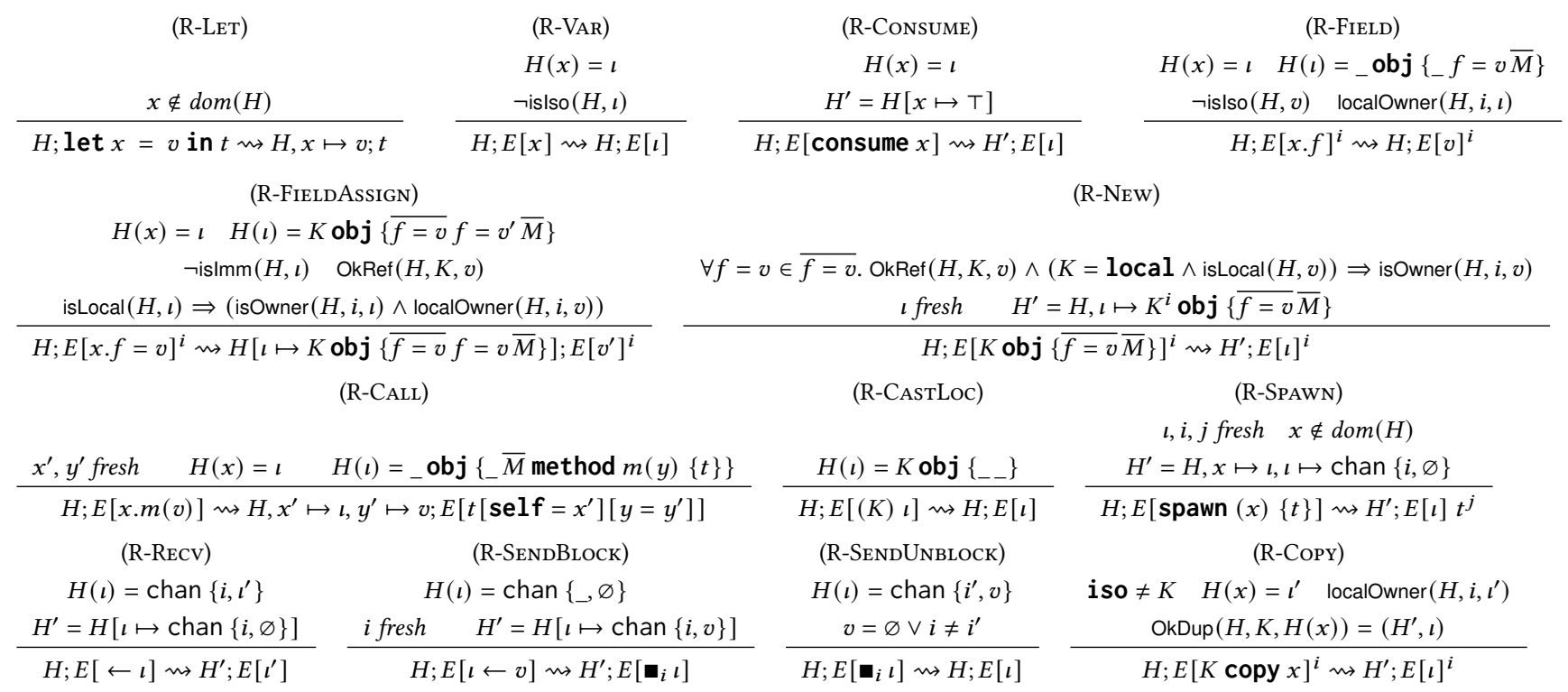

Figure 8. Runtime semantics

An object literal (R-New) checks that values of its fields do not violate the structural constraints imposed by its capability $K$, and if $K$ is local then the current thread must own the local fields. R-NEw returns the (fresh) location of the object.

Calling a method on an object referenced by $x$ and with argument $v$ reduces to the body $t$ of the method with self substituted for a fresh variable bound to the location of $x$ and the singular argument substituted for a fresh variable bound to $v$ (R-CALL).

A new thread is introduced by a spawn operator which introduces a new channel connecting the spawned thread with its "parent" (R-SpAwn). Rules (R-SENDBLOCK), (R-SENDUNBLOCK) and (R-RECV) handle sending and receiving values on a channel. Sending on a channel $\iota$ blocks until the channel is empty, and subsequently blocks the sending thread until the value has been received on the other side. Reading on a channel blocks until there is a value that can be retrieved.

Casting an object (E-CAsTLoc) checks that the object has the specified capability, throwing a permission error, otherwise. The function R-Copy deep copies the object pointed by $\iota$, returning a heap that contains the copy of the object graph with capability $K$ and a fresh location that points to the object copied. ${ }^{10}$ The helper functions used above are defined thus:

$\frac{(\operatorname{ReF} \text { CHeck })}{H(\iota)=K^{\prime} \text { obj }\left\{\left\{_{-}\right\} \quad K \leq K^{\prime}\right.}$

(Helper-LocalOwner)

isLocal $(H, v) \Rightarrow$ isOwner $(H, i, v)$

localOwner $(H, i, v)$

\footnotetext{
${ }^{10}$ The helper function $\operatorname{OkDup}(H, K, v)$ is a standard deep-copying operation. $[17,52]$
}

For simplicity, we have gathered some rules that trap capability errors at run-time in Fig. 9. Common errors when accessing non-existent fields and methods throw a $\operatorname{Err}_{N}$ error (e.g., E-NoSuchFiELD). Accessing values which are absent due to a destructive read yields a $\operatorname{Err}_{A}$ (e.g., E-ConsumE). Assigning an illegal value to a field is not allowed (e.g., EAliasIso and E-IsoField). Casts to the wrong capability reduce to $\operatorname{Err}_{C}$. (Remaining rules in Appendix A, Fig. 12.)

\subsection{Well-Formedness}

We define the environment (also used as store typing [62]) as $\Gamma::=\epsilon|\Gamma, x: K| \Gamma, \iota: K$, where $\epsilon$ represents the empty environment, and $x: K$ and $\iota: K$ mean variable $x$ and location $\iota$ have capability $K$.

(WF-ENV-EMPTy)

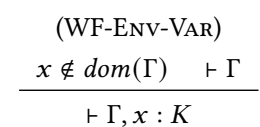

(WF-ENV-Loc)

$\vdash \epsilon$

$\vdash \Gamma, x: K$

$\frac{\iota \notin \operatorname{dom}(\Gamma) \quad \vdash \Gamma}{\vdash \Gamma, \iota: K}$

Well-formedness rules are mostly standard and straightforward. Objects' thread-locality and proper isolation (for objects with local and isolated capabilities respectively) fall out of well-formedness and appear in the Appendix. A wellformed configuration $H$; $\Gamma \vdash \bar{T}$ (WF-CONFIGURATION) consists of predicates establishing that local objects (in the heap) cannot be reachable from multiple threads and that isolated objects (in the heap) have a single reference modulo borrowing (Appendix, Definitions B.8 and B.9). Essentially, the well-formed rules guarantee that the heap is well-formed w.r.t. object capability (and its fields) and that variables are not duplicated when introduced, but they do not statically forbid violation of object capabilities (which will throw a 


\begin{tabular}{|c|c|c|c|c|}
\hline \multirow{2}{*}{ (E-NoSuChFIELD) } & \multirow[t]{2}{*}{ (E-Consume) } & \multirow{2}{*}{$\begin{array}{c}\text { (E-AliasIso) } \\
H(x)=\iota\end{array}$} & (E-IsoFIELD) & \multirow{3}{*}{$\begin{array}{c}\text { (E-CASTERRoR) } \\
H(\iota)=K^{\prime} \text { obj }\left\{{ }_{--}\right\} \\
K^{\prime} \neq K\end{array}$} \\
\hline & & & $H(x)=\iota^{\prime} \quad H(x . f)=\iota$ & \\
\hline$H(x . f)=\perp$ & $H(x)=\top$ & islso $(H, \iota)$ & localOwner $\left(H, i, \iota^{\prime}\right) \quad$ islso $(H, \iota)$ & \\
\hline$H ; E[x . f] \leadsto H ; \operatorname{Err}_{N}$ & $H ; E[$ consume $x] \leadsto H ; \operatorname{Err}_{A}$ & $H ; E[x] \leadsto H ; \operatorname{Err}_{P}$ & $H ; E[x . f]^{i} \leadsto H ; \operatorname{Err}_{P}$ & $H ; E[(K) \iota] \leadsto H ; \operatorname{Err}_{C}$ \\
\hline
\end{tabular}

Figure 9. Expression rules producing errors. To reduce clutter, we write $H(x . f)=v$ when $H(x)=\iota \wedge H(\iota)={ }_{-}$obj $\left\{F_{-}\right\}$and $f=v \in F$. (Remaining rules in Appendix A, Fig. 12.)

permission run-time error).

\begin{tabular}{|c|c|c|c|}
\hline \multicolumn{3}{|c|}{ (WF-Configuration) } & \multirow[t]{2}{*}{ (WF-TERM) } \\
\hline $\operatorname{dom}(\Gamma)$ & $=\operatorname{dom}(H)$ & $\Gamma \vdash H$ & \\
\hline$\forall t \in \bar{T} . \Gamma \vdash H ; t$ & $\operatorname{Local}(H, \bar{T})$ & Isolated $(H, \bar{T})$ & $\Gamma \vdash H \quad \Gamma \vdash t$ \\
\hline \multicolumn{3}{|c|}{$\Gamma \vdash H ; T$} & $\Gamma \vdash H ; t$ \\
\hline
\end{tabular}

Terms are well-formed (WF-TERM) if the store is well-formed w.r.t. an environment $(\Gamma \vdash H)$ and the term is well-typed $(\Gamma \vdash t)$. The store is well-formed w.r.t. an environment if every variable and location in the store is defined in the environment (WF-H-ABSENT, WF-H-CHAN, and WF-H-VAR), if there is a match between the object capability and the variable's (or location's) expected capability (WF-H-ОвJест), and object fields are compatible with their capability object. The environment is well-formed if there are no duplicate locations or variables (WF-ENV-^ rules).

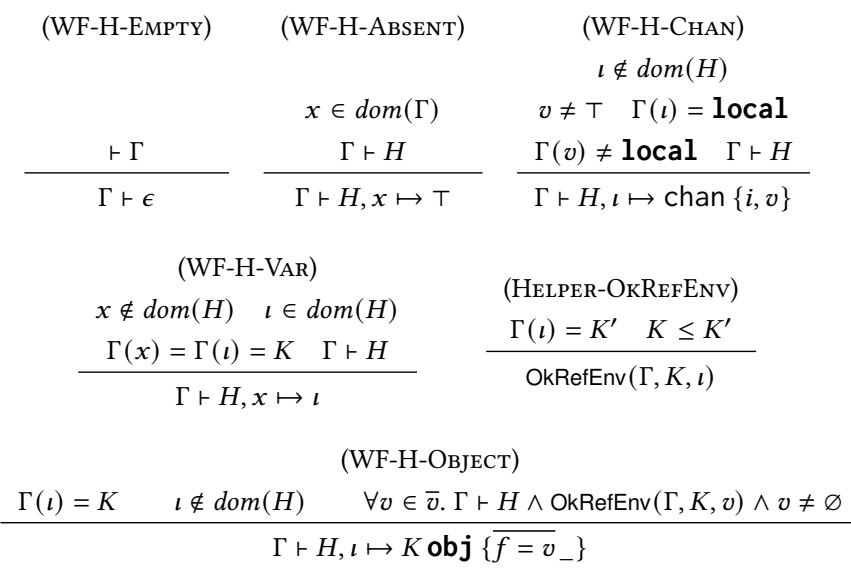

Programs are well-formed w.r.t an environment $\Gamma$ (Fig. 10) when let terms always introduce new variables, and when all accessible variables are defined in $\Gamma$, e.g., WF-VAR.

\subsection{Properties of Well-formed Programs}

We highlight the properties satisfied by well-formed programs (proofs in the Appendix):

- Progress and Preservation (Theorems 5.1 and 5.2). This means that if a well-formed program is not finished (empty state), is not an error (normal, absent, permission, or cast error), or is not in a deadlock state (terminal configuration), then it can take a reduction step until it ends in a terminal configuration state and the result of each reduction step is well-formed.

- Data-Race Freedom (Theorem 5.5). Programs without unsafe objects are data-race free by construction.
- Dynamic Gradual Guarantee (Theorem 5.6). Adaptation of the gradual guarantee $[69,70]$ stating capabilities do not affect the run-time semantics, modulo casts and capability errors. Essentially, if an unsafe program is well-formed and takes a reduction step, the same program with capability annotations either reduces to the same run-time configuration (modulo safe erasure, Appendix B Definition B.11) or throws an error due to a cast or capability violation.

Programs start in an initial well-formed configuration $\epsilon ; t$ (Appendix, Definition B.1), and reduce to new configurations. Progress (Theorem 5.1) guarantees that a well-formed configuration reduces to a new configuration, or it is terminal (Appendix, Definition B.2). From Preservation (Theorem 5.2), a reduction step always leads to a well-formed configuration. Terminal configurations are either finished programs, errors (with $\bar{T} E r r \equiv E r r$ from equivalence rules on Page 10), or a deadlock configuration (Appendix, Definition B.3). A deadlock configuration happens when all threads are either waiting on a receive or on a send operation.

Theorem 5.1 (Progress). A well-formed configuration $\Gamma \vdash$ $H ; \bar{T}$ is either a terminal configuration or $H ; \bar{T} \leadsto H^{\prime} ; \overline{T^{\prime}}$.

Theorem 5.2 (Preservation). If $\Gamma \vdash H ; t \bar{T}$ is a well-formed configuration, and $H ; t \bar{T} \rightsquigarrow H^{\prime} ; \overline{T^{\prime}} \bar{T}$ then, there exists $a \Gamma^{\prime}$ s.t. $\Gamma^{\prime} \supseteq \Gamma$ and $\Gamma^{\prime} \vdash H^{\prime} ; \overline{T^{\prime}} \bar{T}$

Corollary 5.3 (Thread-Affinity of Thread-Local Fields). Implied by Preservation, a thread local object with owner $i$ cannot contain a thread local object with owner $j$, where $i \neq j$. The only way a local object can reference another local object of a different owner is via field assignment (R-FIELDASSIGN). But RFIELDASSIGN checks that target and source share owners. Thus, thread local objects can only reference thread local objects of the same owner.

Definition 5.4 (Data Race). Informally, a data race is defined as two threads accessing (write-write or read-write) the same field without any interleaving synchronisation. In our setting, this translates to two accesses to the same field of an object $o$ in threads $i$ and $j$ without an interleaving explicit transfer of $o$ from $i$ to $j$. Thus, a data race in Dalarna requires the ability of a mutable object to be referenced from two threads at the same time (formal definition in Definition B.4). 


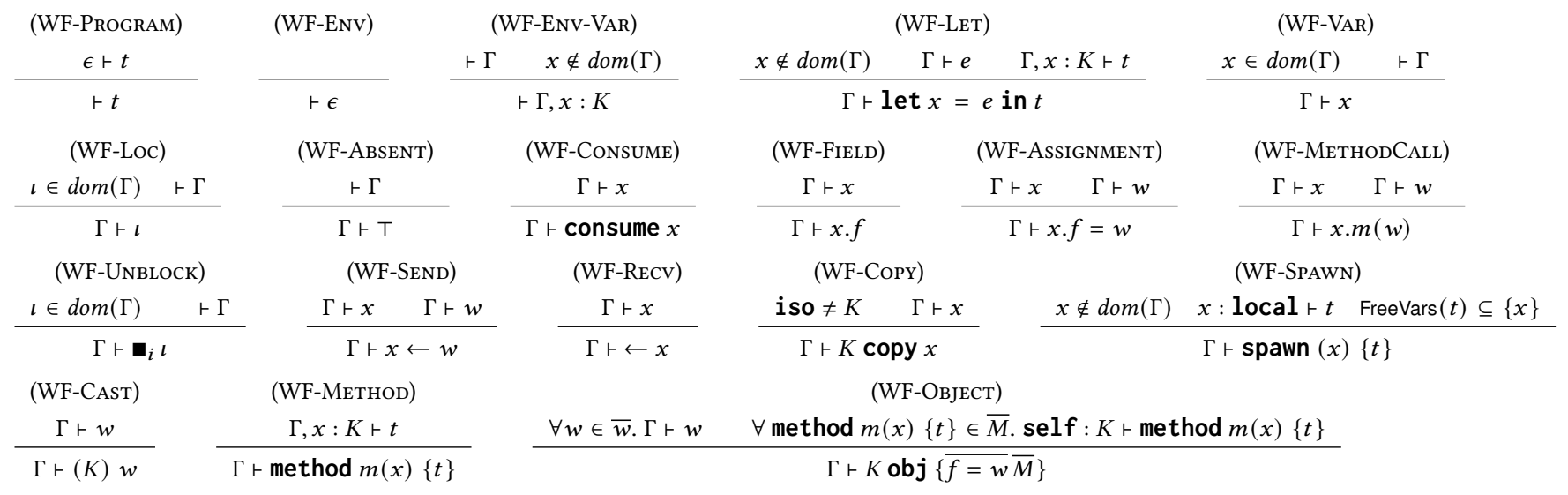

Figure 10. Well-formed declarations, terms and expressions. $\Gamma::=\epsilon|\Gamma, x: K| \Gamma, \iota: K$

Theorem 5.5 states that Dalarna is data race-free modulo unsafe objects. Objects that use safe capabilities cannot introduce a data race; unsafe objects may introduce data races.

Theorem 5.5 (Dalarna is Data-Race Free Modulo Unsafe Objects). All data races directly or indirectly involve an unsafe object. A data race is defined as a read/write or write/write access to an object by different threads without interleaving synchronisation (In our formalism, this means an interleaving transfer of an object to another thread; formal proof in Appendix).

We now proceed to sketch the proof of data-race freedom for safe objects by showing that it is not possible for a mutable safe object to be aliased from two different threads at the same time. We refer to $l, i, j, r_{1}$ and $r_{2}$ from Definition 5.4 for clarity.

Let us examine the implications of $l$ having any of the three safe capabilities (and ignore unsafe objects for now).

1. I is immutable. By E-BAdFieldAssign, attempts to write fields of an immutable object will err. Thus, if $l$ is immutable, then $R$ will contain an error, which it did not by assumption.

2. $l$ is local. By R-Field and R-FieldAssign, attempts to write a field of a local from outside of its creating thread will err. Thus, if $l$ is local, then $R$ will contain an error (because $i \neq j$ ), which it did not by assumption.

3. $l$ is isolated. Fields of isolated objects can be read or written freely. Thus, we must show that $l$ can be accessible in two threads at the same time. In the initial heap, no objects exist that is shared across threads and our only way to share objects across threads is by sending them on a channel. An isolated $l$ can be sent on a channel. However, this requires that $l$ is consumed, meaning it will no longer be accessible by the sender. To avoid the consumption, we could store $l$ in a field of an object, and then transfer the object. Such objects, would have to be immutable or local. However, by R-FieldAssign, immutable or local objects cannot contain isolated objects.

Thus, we cannot create a situation where $l$ is in both $r_{1}$ and $r_{2}$ without any interleaving send.

A program that uses unsafe objects may use these to store local and isolated objects. Thus, an unsafe object $u$ aliased across threads could store a local or isolated $l$ in $u$.f (see 3 . above). This would allow threads $i$ and $j$ to do $u$.f.g. If $l$ is local, unless $i=j$, at least one of the accesses will err (or both if neither $i$ nor $j$ is the creating thread of $l$, see 2. above). If $l$ is isolated, $u . f . g$ will err by E-IsoField. Thus, even in the presence of unsafe objects, safe objects will not participate in data races.

Progress and preservation guarantee the absence of untrapped errors and Theorem 5.5 shows that all data races can be blamed on unsafe objects. Next, we show that capability annotations do not affect the run-time semantics, modulo cast or capability violations which trap operations that if allowed could lead to a data race. We show this in the Dynamic Gradual Guarantee theorem (Theorem 5.6, adapted from [70]). We unpack this theorem before stating it formally. Let $P$ be a well-formed program, and $S$ its "stripped equivalent", where all safe capabilities have been erased (and thus replaced with unsafe). Below, "reduces" denotes a single reduction step.

1. The well-formedness of $S$ follows from the well-formedness of $P$, as an unsafe object can reference any object (Appendix B Definition B.11).

2. If $P$ reduces to a non-error configuration, $S$ reduces to the same configuration and the same heap (modulo heap capability erasure); in case $P$ throws an error caused by absent values or normal errors, $S$ will throw the same error. The two evaluations only diverge if $P$ throws a permission error in a dynamic check - this will never happen in $S$ because it does not have any safe capabilities. (See Lemma B.27 e.g., 
E-BADFiELDAsSign or E-BADINSTANTIATION among others.)

3. If $S$ reduces to an error configuration, $P$ will reduce to the same error configuration. If $S$ reduces to a nonerror configuration, $P$ will either reduce to the same configuration (modulo capability stripping), or throw a capability or permission error.

Theorem 5.6 (Dynamic Gradual Guarantee). Let $H ; t \overline{T_{0}}$ be a configuration and $\Gamma$ a store type such that $\Gamma \vdash H ; t \overline{T_{0}}$. Let $e$ be a function that replaces safe capabilities with unsafe in heaps, terms, etc (Definition B.11). Then:

$$
\begin{aligned}
& \text { 1. } \Gamma^{e} \vdash\left(H ; t \overline{T_{0}}\right)^{e} \text {. } \\
& \text { 2. a. If } H ; t \overline{T_{0}} \leadsto H^{\prime} ; \overline{T_{1}} \overline{T_{0}} \text { and } \overline{T_{1}} \neq \text { Err then, } \\
& \left(H ; t \overline{T_{0}}\right)^{e} \rightsquigarrow\left(H^{\prime} ; \overline{T_{1}} \overline{T_{0}}\right)^{e} \text {. } \\
& \text { b. If } H ; t \overline{T_{0}} \leadsto H^{\prime} ; \overline{T_{1}} \overline{T_{0}} \text { and } \overline{T_{1}}=\operatorname{Err}_{A} \vee \operatorname{Err}_{N} \text { then, } \\
& (H ; t \bar{T})^{e} \rightsquigarrow H^{\prime \prime} ; \overline{T_{1}} \overline{T_{0}} \\
& \text { 3. a. If }\left(H ; t \overline{T_{0}}\right)^{e} \rightsquigarrow\left(H^{\prime} ; \operatorname{Err} \overline{T_{0}}\right)^{e} \text { then, } \\
& H ; t \overline{T_{0}} \rightsquigarrow H^{\prime} ; \operatorname{Err} \overline{T_{0}} \\
& \text { b. If }\left(H ; t \overline{T_{0}}\right)^{e} \leadsto\left(H^{\prime} ; \bar{T} \overline{T_{0}}\right)^{e} \text { and } \bar{T} \neq \text { Err then, } \\
& H ; t \overline{T_{0}} \leadsto H^{\prime} ; \overline{T^{\prime}} \overline{T_{0}} \text { and } \overline{T^{\prime}}=E_{E r r_{P} \vee E_{C r}} \vee \bar{T} \text {. }
\end{aligned}
$$

The Dynamic Gradual Guarantee (Theorem 5.6) uses a single step reduction to guarantee that the capabilities are semantics preserving, modulo permission and cast errors. We extend the Dynamic Gradual Guarantee to account for multi-step reductions, starting from an initial configuration until reaching a terminal configuration, i.e., $\epsilon ; P \rightsquigarrow{ }^{*} H$;C. To remove non-determinism of program reductions, we define the trace of a program as a list of pairs that contain the reduction step and the thread id on which the reduction happens. We extend the reduction relation to account for the trace, named the replay reduction relation, which is the standard reduction relation except that it deterministically applies the expected reduction step on the expected thread id (Appendix, Definitions B.5 to B.7 and Theorem B.28). The basic idea is to reduce a safe program to a terminal configuration, which produces a trace. We use this trace to replay the reductions on the capability stripped (unsafe) program (and vice versa), showing two programs reduce to the same terminal configuration modulo cast errors and permission errors. Since it obscures some cases where the identical step is taken, we show the single-step theorem in the paper which highlights these cases.

\section{Daddala: A Prototype Implementation of Dala in Grace}

To be able to explore the applicability of our capability model, we have embedded it within a preexisting general-purpose object-oriented language, Grace [3]. We extended an existing implementation to support run-time enforcement of capabilities with the correct dynamic semantics. Programs in the formal syntax have a straightforward translation into the
Grace syntax, and the wider features of the language are also usable with minimal limitations.

Our implementation, Daddala ${ }^{11}$, is fully embedded within the existing Grace syntax, with no syntactic changes to the host language. The embedded syntax will be familiar to the formal syntax above.

Objects in Grace are created with object literals object $\{\ldots\}$, which instantiate new anonymous objects with the given methods, fields, and inline initialisation code. To specify the capability of the object we use the existing inheritance system $[44,59]$ and write object \{use isolate\} (or immutable, local; unsafe is the default).

let (e) in $\{x \rightarrow t\}$, spawn $\{x \rightarrow t\}$, and consume $(x)$ perform the corresponding roles. Send and receive is $c \leftarrow 5$ and $\leftarrow$ c. Casting is implemented straightforwardly as Grace type annotations precede the capability name: $\operatorname{var} \mathrm{x}:$ iso $:=\mathrm{y}$.

\subsection{Extending Grace with Dala Capabilities}

Our prototype extends Grace in two ways: every assignment performs some check and objects are tagged at creation with their capabilities. Objects are tagged unsafe (default), immutable, local, free isolate, or bound isolate.

An isolate is free if it is not currently stored in a variable or field, otherwise bound. These are the only two capability tags that are not fixed for the object's lifetime (distinguishing this system from Grace's brands [43], which are immutable). When a variable or field containing a (bound) isolate is assigned to, the assignment re-tags the object as a free isolate. When any storage location is assigned to, the assignment checks the right-hand side and raises an exception if it is a bound isolate (as this would otherwise violate alias-freedom), or re-tags a free isolate as a bound isolate before storing it. In essence, this pair behaves as a one-bit reference count for isolate objects.

Assignments occur at assignment statements $(x:=y)$, variable and field declarations ( $\operatorname{var} x:=y$, def $x=y)$, binding of method parameters (method $f \circ o(x)\{\}$; foo $(y))$, and binding of block (first-class lambda) parameters $(\{x \rightarrow\}$. apply $(y))$. All of these locations perform the free/bound isolate handling above, while fields also perform the necessary structural checks based on the capability of their enclosing object and of the assigned object. These are the only places new kinds of error have been introduced.

There is one special extension motivated by simplicity: the consume $(x)$ operation is syntactically rewritten to $(x:=$ nul1), rather than truly a method as the syntax suggests.

\subsection{Limitations Due to Embedding}

Some features of the Grace language run counter to the properties of the formal model. While a program written according to the model will execute correctly, it is possible to construct pathological programs using other language

\footnotetext{
${ }^{11}$ Daddala is a genus of moth in the family Erebidæ.
} 


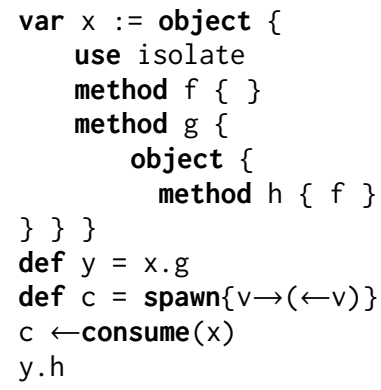

Figure 11. Limitations due to embedding: current unsoundness of our implementation. See $§ 6.2$.

features that violate the imposed constraints in the present implementation. In particular, Grace makes heavy use of lexical capture: all scopes are closures, including object bodies [9]. A program such as the one in Fig. 11 will access the isolate object that should no longer be reachable, because the $y$ object retains the lexical scope of the surrounding object and can implicitly call its method f. Our prototype will not raise an error at this point. This type of capture is unavoidable without breaking fundamental aspects of the language (which uses scoping to provide even builtins like if-then-else [41]), but the use of improperly-captured objects can be detected statically. While it is therefore possible to eliminate these errors (and other implementation strategies, such as proxies for isolates, can similarly address the issue), our current prototype permits a programmer who goes far outside the model to shoot themselves in the foot.

\subsection{Concluding Remarks}

Extending an existing system to support capabilities in Dala style is relatively straightforward; it is only the various kinds of variable assignment that require meaningful intervention, plus a small object tag. We modified an implementation that was previously designed with no concept of capabilities, so that it now enforces them dynamically and supports a direct translation from the formal model.

\section{Related Work}

The Dala capability model (immutables $<$ isolates $<$ threadlocals) carefully selects a number of well-known concepts from the literature $[17,20,40,50,57,75]$. Dala's key contribution here is the careful combination: what we have left out is at least as important as the features we have included. Given that an actor is essentially a thread plus thread-local storage [26], our distinctions are similar to many object-actor hybrid systems [23, 28, 36, 46, 47, 67], although, crucially, we follow Singularity [49] by incorporating isolates for fast transfers. Similarly, there are many more flexible models for distinguishing between read-only and read-write objects: we adopt "deep immutability" for its clear conceptual model
Glacier [22]. While there are certainly more complicated models of permissions and capabilities for data-race freedom (e.g., [13, 17, 20, 77] and many others), we consider our chosen set of concepts a "sweet spot" in the balance between expressivity and complexity.

\subsection{Capabilities and Ownership}

Dala is also heavily influenced by static capability-based programming languages [8, 13, 19, 20,35]. Capability-based programming languages require all programs to be fully annotated with capabilities, and these annotations guarantee data-race freedom, with erasure semantics. In contrast, our approach begins with a dynamic language that allows data race and data race-free programs to interact, and we maintain data race-freedom for programs with safe capabilities.

Sergey and Clarke [68] add gradual ownership to a static language, introducing notions of parametric ownership and inserting casts when needed; they prove soundness and common ownership invariants. Our work has similarities in that isolated objects can be seen as owners-as-dominators, and our local objects have threads as owners. Dala differs in that it is a dynamic, concurrent language, and we prove common invariants and data race-freedom for safe objects.

HJp [72] enforces safe sharing of objects using a permissionbased gradual typing, which inserts run-time checks when necessary. Objects are either in shared-read permission, which allows reading from multiple threads but no mutation, or read-write permission, allows any mutation and aliasing but no sharing. It also introduces storable permissions which allows a permission to refer to a tree of objects. Our approach uses capabilities at run-time; imm capabilities are similar to HJp shared-read and read-write permissions are similar to local. In addition, Dala also has the concept of iso which can move across threads but do not allow any aliasing.

Roberts et al. [63] (and recently [58]) showed that run-time gradual type checks could have minimal or no performance impact on a suitable virtual machine, despite what is naively much extra checking for partially-typed programs. We chose to extend their Moth virtual machine for our dynamicallychecked implementation to take advantage of their work.

\subsection{Capabilities In The Wild}

Castegren's et al work [13] seems to be the first one where reference capabilities are orthogonal to the concurrency model i.e., the reference capabilities seem applicable to multiple concurrency models. Their capabilities have been formalised using fork-join style but their implementation uses active objects [8]. The implementation of Gordon's et al reference immutability work closely follows the formal semantics; because their reference permissions apply transitively to fields of the object, it is not clear whether the model is general enough to be applicable to different concurrency models, e.g., actor or tuplespace model [25, 34]. Boyland et 
al's work [5] can encode 8 object capabilities to express different invariants and they argue that these can be used in concurrent programs with no run-time cost, when programs are fully typed. We believe the capability system is expressive enough to work on different concurrency models, but it is not clear whether their capabilities enforce data race freedom. In languages such as E [56], AmbientTalk [23], and Newspeak [6] references (far and near) represent object capabilities [29] and use a vat-based concurrency model. In contrast, our work is simple and uses 3 capabilities, allows interoperation between safe and "racy" programs and (as far as we know) it is the first one to use reference capabilities in a dynamic language where the capabilities are orthogonal to the concurrency model.

\subsection{Race Detectors}

Although our capability checks guarantee data-race freedom, they are different to the checks that a data-race detector might employ [66]. These checks are also in some sense "eager" or may cause false positives. For example, a program that effectively transfers a mutable object $o$ between two threads will execute without errors if $o$ is isolated, but not if it is local and the non-owning thread dereferences it. This is a somewhat pragmatic choice, but guided by our desire to make our capabilities a tool for programmers to capture their intent. Thus, we expect that a local object is explicitly demarcated local (at creation time) and not isolated for a reason. Thus, Dala helps programmers state their intentions and check that the programs they write conform to said intentions. This is different from a data race detector which may only fire if a data race occurs (which may happen on some runs but not others of the same program).

\section{Discussion and Future Work}

Our claim of efficiency rests on absence of deep copying and turning local accesses into asynchronous operations. That said, our capabilities incur a cost on (most) accesses to objects - e.g., on writes to fields, etc. To remove most of this cost will require self-optimising run-times [73] and techniques similar to Grace's transient checks [63] to reduce the number of checks needed to satisfy the capability invariants.

Adding gradual capabilities at the type level will allow most checks to be removed [58, 63], but more importantly help programmers document the behaviour of code. Notably this addition will not need escape hatches due to inflexible types as programmers can fall-back to dynamic checks which are equally safe.

In this paper, the Dala capabilities only constrain heap structures. Nothing prevents a stack variable in an immutable object to point to an unsafe object. To reason about datarace freedom of a method call on a safe object, we need to consider the methods arguments' capabilities. Extending the structural constraints to stack variables is an interesting point in the design space: if immutable objects can only "see" other immutable objects, method calls on immutables are guaranteed to be side-effect free modulo allocation and GC. For isos, side-effects are not possible, but preexisting objects may be updated in place. Finally, local objects would only observe local objects belonging to the same thread (which is probably very desirable), and permit side-effects visible in the current thread only. Such a design can reduce the number of checks (e.g., all checks of thread-ownership happen only when calling a local method in an unsafe context).

\section{Conclusion}

A data race is a fundamental, low-level aspect of a program which is not tied to the intended semantics of a particular application. While many race conditions stem from data-races, data-race freedom does not mean absence of race conditions. Data-race freedom is however still important: removing them removes many race conditions and moreover makes a program's semantics independent on the idiosyncrasies of a particular (weak) memory model. In the case of programming languages like $\mathrm{C}$ and $\mathrm{C}++$, data-races are examples undefined behaviour. The Dala capabilities guarantee absence of data races in safe objects by imposing restrictions on all code that interacts with these objects. Safe and unsafe objects can co-exist and the presence of the latter does not compromise the guarantees of the former.

Dala helps programmers structure their programs with capabilities including immutable, isolated, and thread-local. We support the Dala design with a formal model, clear and proven properties w.r.t. data-race freedom and semantics preservation when capabilities are added to a program.

We provide Daddala $(\S 6)$, an early proof-of-concept prototype implementation which is available as open source. ${ }^{12}$ Based on this last experience, we believe that our model can provide opt-in data-race safety to programmers on top of existing languages, with relatively little implementation difficulty and overhead.

\section{References}

[1] [n.d.]. Akka Documentation. Actor Best Practices. https://doc.akka.io/ docs/akka/current/general/actor-systems.html\#actor-best-practices. Accessed August 26, 2020.

[2] Joe Armstrong, Robert Virding, and Mike Williams. 1993. Concurrent programming in ERLANG. Prentice Hall.

[3] Andrew P. Black, Kim B. Bruce, Michael Homer, and James Noble. 2012. Grace: the absence of (inessential) difficulty. In ACM Symposium on New Ideas in Programming and Reflections on Software, Onward! 2012, part of SPLASH '12, Tucson, AZ, USA, October 21-26, 2012, Gary T. Leavens and Jonathan Edwards (Eds.). ACM, 85-98. https://doi.org/10. 1145/2384592.2384601

[4] John Boyland. 2001. Alias burying: Unique variables without destructive reads. Softw. Pract. Exp. 31, 6 (2001), 533-553. https: //doi.org/10.1002/spe.370

\footnotetext{
${ }^{12}$ https://github.com/gracelang/moth-SOMns/tree/daddala
} 
[5] John Boyland, James Noble, and William Retert. 2001. Capabilities for Sharing: A Generalisation of Uniqueness and Read-Only. In ECOOP 2001 - Object-Oriented Programming, 15th European Conference, Budapest, Hungary, June 18-22, 2001, Proceedings (Lecture Notes in Computer Science, Vol. 2072), Jørgen Lindskov Knudsen (Ed.). Springer, 2-27. https://doi.org/10.1007/3-540-45337-7_2

[6] Gilad Bracha. 2017. Newspeak programming language draft specification version 0.1. Technical Report. Technical report, Ministry of Truth.

[7] Gilad Bracha, Peter von der Ahé, Vassili Bykov, Yaron Kashai, William Maddox, and Eliot Miranda. 2010. Modules as Objects in Newspeak, See [30], 405-428. https://doi.org/10.1007/978-3-642-14107-2_20

[8] Stephan Brandauer, Elias Castegren, Dave Clarke, Kiko FernandezReyes, Einar Broch Johnsen, Ka I Pun, Silvia Lizeth Tapia Tarifa, Tobias Wrigstad, and Albert Mingkun Yang. 2015. Parallel Objects for Multicores: A Glimpse at the Parallel Language Encore. In Formal Methods for Multicore Programming - 15th International School on Formal Methods for the Design of Computer, Communication, and Software Systems, SFM 2015, Bertinoro, Italy, Fune 15-19, 2015, Advanced Lectures (Lecture Notes in Computer Science, Vol. 9104), Marco Bernardo and Einar Broch Johnsen (Eds.). Springer, 1-56. https://doi.org/10.1007/978-3-319-18941 31

[9] Kim Bruce, Andrew Black, Michael Homer, James Noble, Amy Ruskin, and Richard Yannow. 2013. Seeking Grace: a new object-oriented language for novices. In SIGCSE.

[10] Denis Caromel, Christian Delbé, Alexandre Costanzo, and Mario Leyton. 2006. ProActive: an Integrated platform for programming and running applications on Grids and P2P systems. Computational Methods in Science and Technology 12 (01 2006). https://doi.org/10.12921/cmst. 2006.12.01.69-77

[11] Denis Caromel, Ludovic Henrio, and Bernard P. Serpette. 2009. Asynchronous sequential processes. Inf. Comput. 207, 4 (2009), 459-495. https://doi.org/10.1016/j.ic.2008.12.004

[12] Elias Castegren, Joel Wallin, and Tobias Wrigstad. 2018. Bestow and atomic: Concurrent programming using isolation, delegation and grouping. Journal of Logical and Algebraic Methods in Programming 100 (2018), 130 - 151. https://doi.org/10.1016/j.jlamp.2018.06.007

[13] Elias Castegren and Tobias Wrigstad. 2016. Reference Capabilities for Concurrency Control. In 30th European Conference on ObjectOriented Programming, ECOOP 2016, fuly 18-22, 2016, Rome, Italy (LIPIcs, Vol. 56), Shriram Krishnamurthi and Benjamin S. Lerner (Eds.) Schloss Dagstuhl - Leibniz-Zentrum fuer Informatik, 5:1-5:26. https: //doi.org/10.4230/LIPIcs.ECOOP.2016.5

[14] Dominik Charousset, Raphael Hiesgen, and Thomas C. Schmidt. 2014 $\mathrm{CAF}$ - the $\mathrm{C}++$ Actor Framework for Scalable and Resource-Efficient Applications. In Proceedings of the 4th International Workshop on Programming based on Actors Agents \& Decentralized Control, AGERE! 2014, Portland, OR, USA, October 20, 2014, Elisa Gonzalez Boix, Philipp Haller, Alessandro Ricci, and Carlos Varela (Eds.). ACM, 15-28. https: //doi.org/10.1145/2687357.2687363

[15] Dave Clarke, Johan Östlund, Ilya Sergey, and Tobias Wrigstad. 2013. Ownership Types: A Survey. In Aliasing in Object-Oriented Programming. Types, Analysis and Verification, Dave Clarke, James Noble, and Tobias Wrigstad (Eds.). Lecture Notes in Computer Science, Vol. 7850. Springer, 15-58. https://doi.org/10.1007/978-3-642-36946-9_3

[16] Dave Clarke and Tobias Wrigstad. 2003. External Uniqueness Is Unique Enough. In ECOOP 2003 - Object-Oriented Programming, 17th European Conference, Darmstadt, Germany, Fuly 21-25, 2003, Proceedings (Lecture Notes in Computer Science, Vol. 2743), Luca Cardelli (Ed.). Springer, 176-200. https://doi.org/10.1007/978-3-540-45070-2_9

[17] Dave Clarke, Tobias Wrigstad, Johan Östlund, and Einar Broch Johnsen. 2008. Minimal Ownership for Active Objects. In Programming Languages and Systems, 6th Asian Symposium, APLAS 2008, Bangalore, India, December 9-11, 2008. Proceedings (Lecture Notes in Computer Science, Vol. 5356), G. Ramalingam (Ed.). Springer, 139-154. https: //doi.org/10.1007/978-3-540-89330-1_11

[18] David G. Clarke and Sophia Drossopoulou. 2002. Ownership, encapsulation and the disjointness of type and effect. In Proceedings of the 2002 ACM SIGPLAN Conference on Object-Oriented Programming Systems, Languages and Applications, OOPSLA 2002, Seattle, Washington, USA, November 4-8, 2002, Mamdouh Ibrahim and Satoshi Matsuoka (Eds.). ACM, 292-310. https://doi.org/10.1145/582419.582447

[19] Sylvan Clebsch and Sophia Drossopoulou. 2013. Fully concurrent garbage collection of actors on many-core machines. In Proceedings of the 2013 ACM SIGPLAN International Conference on Object Oriented Programming Systems Languages \& Applications, OOPSLA 2013, part of SPLASH 2013, Indianapolis, IN, USA, October 26-31, 2013, Antony L. Hosking, Patrick Th. Eugster, and Cristina V. Lopes (Eds.). ACM, 553570. https://doi.org/10.1145/2509136.2509557

[20] Sylvan Clebsch, Sophia Drossopoulou, Sebastian Blessing, and Andy McNeil. 2015. Deny capabilities for safe, fast actors. In Proceedings of the 5th International Workshop on Programming Based on Actors, Agents, and Decentralized Control, AGERE! 2015, Pittsburgh, PA, USA, October 26, 2015, Elisa Gonzalez Boix, Philipp Haller, Alessandro Ricci, and Carlos Varela (Eds.). ACM, 1-12. https://doi.org/10.1145/2824815.2824816

[21] Sylvan Clebsch, Juliana Franco, Sophia Drossopoulou, Albert Mingkun Yang, Tobias Wrigstad, and Jan Vitek. 2017. Orca: GC and type system co-design for actor languages. Proc. ACM Program. Lang. 1, OOPSLA (2017), 72:1-72:28. https://doi.org/10.1145/3133896

[22] Michael J. Coblenz, Whitney Nelson, Jonathan Aldrich, Brad A. Myers, and Joshua Sunshine. 2017. Glacier: transitive class immutability for Java. In Proceedings of the 39th International Conference on Software Engineering, ICSE 2017, Buenos Aires, Argentina, May 20-28, 2017. IEEE / ACM, 496-506. https://doi.org/10.1109/ICSE.2017.52

[23] Tom Van Cutsem, Elisa Gonzalez Boix, Christophe Scholliers, Andoni Lombide Carreton, Dries Harnie, Kevin Pinte, and Wolfgang De Meuter. 2014. AmbientTalk: programming responsive mobile peerto-peer applications with actors. Comput. Lang. Syst. Struct. 40, 3-4 (2014), 112-136. https://doi.org/10.1016/j.cl.2014.05.002

[24] Tom Van Cutsem, Stijn Mostinckx, and Wolfgang De Meuter. 2009. Linguistic symbiosis between event loop actors and threads. Comput. Lang. Syst. Struct. 35, 1 (2009), 80-98. https://doi.org/10.1016/j.cl.2008.06. 005

[25] Frank S. de Boer, Vlad Serbanescu, Reiner Hähnle, Ludovic Henrio, Justine Rochas, Crystal Chang Din, Einar Broch Johnsen, Marjan Sirjani, Ehsan Khamespanah, Kiko Fernandez-Reyes, and Albert Mingkun Yang. 2017. A Survey of Active Object Languages. ACM Comput. Surv. 50, 5 (2017), 76:1-76:39. https://doi.org/10.1145/3122848

[26] Mattias De Wael, Stefan Marr, Bruno De Fraine, Tom Van Cutsem, and Wolfgang De Meuter. 2015. Partitioned Global Address Space Languages. Comput. Surveys 47, 4, Article 62 (June 2015), 27 pages.

[27] J. Dedecker, T. Van Cutsem, S. Mostinckx, T. D'Hondt, and W. De Meuter. 2006. Ambient-Oriented Programming in AmbientTalk. In ECOOP. 230-254.

[28] Jessie Dedecker, Tom Van Cutsem, Stijn Mostinckx, Theo D’Hondt, and Wolfgang De Meuter. 2006. Ambient-Oriented Programming in AmbientTalk. In ECOOP 2006 - Object-Oriented Programming, 20th European Conference, Nantes, France, July 3-7, 2006, Proceedings (Lecture Notes in Computer Science, Vol. 4067), Dave Thomas (Ed.). Springer, 230-254. https://doi.org/10.1007/11785477_16

[29] Jack B. Dennis and Earl C. Van Horn. 1966. Programming semantics for multiprogrammed computations. Commun. ACM 9, 3 (1966), 143-155. https://doi.org/10.1145/365230.365252

[30] Theo D'Hondt (Ed.). 2010. ECOOP 2010 - Object-Oriented Programming, 24th European Conference, Maribor, Slovenia, June 21-25, 2010. Proceedings. Lecture Notes in Computer Science, Vol. 6183. Springer. https://doi.org/10.1007/978-3-642-14107-2

[31] Werner Dietl, Sophia Drossopoulou, and Peter Müller. 2007. Generic Universe Types. In ECOOP 2007 - Object-Oriented Programming, 21st 
European Conference, Berlin, Germany, fuly 30 - August 3, 2007, Proceedings (Lecture Notes in Computer Science, Vol. 4609), Erik Ernst (Ed.). Springer, 28-53. https://doi.org/10.1007/978-3-540-73589-2_3

[32] Darach Ennis. 2005. My Little Pony. At CodeMesh 2015. https://cdn. rawgit.com/darach/my_little_pony/master/my-little-pony.html.

[33] Cormac Flanagan, Amr Sabry, Bruce F. Duba, and Matthias Felleisen. 1993. The Essence of Compiling with Continuations. In Proceedings of the ACM SIGPLAN'93 Conference on Programming Language Design and Implementation (PLDI), Albuquerque, New Mexico, USA, fune 23-25, 1993, Robert Cartwright (Ed.). ACM, 237-247. https://doi.org/10.1145/ 155090.155113

[34] David Gelernter. 1985. Generative Communication in Linda. ACM Trans. Program. Lang. Syst. 7, 1 (1985), 80-112. https://doi.org/10.1145/ 2363.2433

[35] Colin S. Gordon, Matthew J. Parkinson, Jared Parsons, Aleks Bromfield, and Joe Duffy. 2012. Uniqueness and reference immutability for safe parallelism. In Proceedings of the 27th Annual ACM SIGPLAN Conference on Object-Oriented Programming, Systems, Languages, and Applications, OOPSLA 2012, part of SPLASH 2012, Tucson, AZ, USA, October 21-25, 2012, Gary T. Leavens and Matthew B. Dwyer (Eds.). ACM, 21-40. https://doi.org/10.1145/2384616.2384619

[36] Olivier Gruber and Fabienne Boyer. 2013. Ownership-Based Isolation for Concurrent Actors on Multi-core Machines. In ECOOP 2013 - ObjectOriented Programming - 27th European Conference, Montpellier, France, fuly 1-5, 2013. Proceedings (Lecture Notes in Computer Science, Vol. 7920), Giuseppe Castagna (Ed.). Springer, 281-301. https://doi.org/10.1007/9783-642-39038-8_12

[37] Philipp Haller and Alexander Loiko. 2016. LaCasa: lightweight affinity and object capabilities in Scala. In Proceedings of the 2016 ACM SIGPLAN International Conference on Object-Oriented Programming, Systems, Languages, and Applications, OOPSLA 2016, part of SPLASH 2016, Amsterdam, The Netherlands, October 30 - November 4, 2016, Eelco Visser and Yannis Smaragdakis (Eds.). ACM, 272-291. https://doi.org/10.1145/2983990.2984042

[38] Philipp Haller and Martin Odersky. 2010. Capabilities for Uniqueness and Borrowing, See [30], 354-378. https://doi.org/10.1007/978-3-64214107-2_17

[39] Douglas E. Harms and Bruce W. Weide. 1991. Copying and Swapping: Influences on the Design of Reusable Software Components. IEEE Trans. Softw. Eng. 17, 5 (May 1991), 424-435. https://doi.org/10.1109/32. 90445

[40] John Hogg. 1991. Islands: Aliasing Protection in Object-Oriented Languages. In Conference on Object-Oriented Programming Systems, Languages, and Applications (OOPSLA'91), Sixth Annual Conference, Phoenix, Arizona, USA, October 6-11, 1991, Proceedings, Andreas Paepcke (Ed.). ACM, 271-285. https://doi.org/10.1145/117954.117975

[41] Michael Homer, Timothy Jones, James Noble, Kim B Bruce, and Andrew P Black. 2014. Graceful dialects. In ECOOP (LNCS, Vol. 8586), Richard Jones (Ed.). Springer, 131-156.

[42] Einar Broch Johnsen, Reiner Hähnle, Jan Schäfer, Rudolf Schlatte, and Martin Steffen. 2012. ABS: A Core Language for Abstract Behavioral Specification. In Formal Methods for Components and Objects, Bernhard K. Aichernig, Frank S. de Boer, and Marcello M. Bonsangue (Eds.). Springer Berlin Heidelberg, Berlin, Heidelberg, 142-164.

[43] Timothy Jones, Michael Homer, and James Noble. 2015. Brand Objects for Nominal Typing. In ECOOP.

[44] Timothy Jones, Michael Homer, James Noble, and Kim Bruce. 2016. Object Inheritance Without Classes. In 30th European Conference on Object-Oriented Programming (ECOOP 2016) (Leibniz International Proceedings in Informatics (LIPIcs), Vol. 56), Shriram Krishnamurthi and Benjamin S. Lerner (Eds.). Schloss Dagstuhl-Leibniz-Zentrum fuer Informatik, Dagstuhl, Germany, 13:1-13:26. https://doi.org/10.4230/LIPIcs. ECOOP.2016.13
[45] Steve Klabnik and Carol Nichols. 2019. The Rust Programming Language (Covers Rust 2018). No Starch Press.

[46] Joeri De Koster, Stefan Marr, Tom Van Cutsem, and Theo D'Hondt. 2016. Domains: Sharing state in the communicating event-loop actor model. Comput. Lang. Syst. Struct. 45 (2016), 132-160.

[47] Joeri De Koster, Stefan Marr, Theo D'Hondt, and Tom Van Cutsem. 2015. Domains: Safe sharing among actors. Sci. Comput. Program. 98 (2015), 140-158.

[48] Neel Krishnaswami and Jonathan Aldrich. 2005. Permission-based ownership: encapsulating state in higher-order typed languages. In PLDI '05: Proceedings of the 2005 ACM SIGPLAN conference on Programming language design and implementation, Mary Hall (Ed.). Chicago, IL, USA, 96-106.

[49] James Larus and Galen Hunt. 2010. The Singularity System. Commun. ACM 53, 8 (Aug. 2010), 72-79. https://doi.org/10.1145/1787234.1787253

[50] Douglas Lea. 1999. Concurrent programming in fava. Second Edition: Design Principles and Patterns (2nd ed.). Addison-Wesley Longman Publishing Co., Inc., USA.

[51] Amit A. Levy, Michael P. Andersen, Bradford Campbell, David E. Culler, Prabal Dutta, Branden Ghena, Philip Levis, and Pat Pannuto. 2015. Ownership is theft: experiences building an embedded OS in Rust. In Proceedings of the 8th Workshop on Programming Languages and Operating Systems, PLOS 2015, Monterey, California, USA, October 4, 2015, Shan Lu (Ed.). ACM, 21-26. https://doi.org/10.1145/2818302.2818306

[52] Paley Li, Nicholas Cameron, and James Noble. 2012. Sheep cloning with ownership types. In FOOL 2012: 19th International Workshop on Foundations of Object-Oriented Languages. Citeseer, 59.

[53] Paul Liétar. 2017. Formalizing Generics for Pony. Master's thesis. Imperial College London.

[54] Barbara Liskov and Liuba Shrira. 1988. Promises: Linguistic Support for Efficient Asynchronous Procedure Calls in Distributed Systems. In Proceedings of the ACM SIGPLAN'88 Conference on Programming Language Design and Implementation (PLDI), Atlanta, Georgia, USA, fune 22-24, 1988, Richard L. Wexelblat (Ed.). ACM, 260-267. https: //doi.org/10.1145/53990.54016

[55] Nicholas D. Matsakis and Felix S. Klock II. 2014. The Rust language. In Proceedings of the 2014 ACM SIGAda annual conference on High integrity language technology, HILT 2014, Portland, Oregon, USA, October 1821, 2014, Michael Feldman and S. Tucker Taft (Eds.). ACM, 103-104. https://doi.org/10.1145/2663171.2663188

[56] Mark S. Miller. 2006. Robust Composition: Towards a Unified Approach to Access Control and Concurrency Control. Ph.D. Dissertation. Baltimore, Maryland.

[57] Mark Samuel Miller. 2006. Robust Composition: Towards a Unified Approach to Access Control and Concurrency Control. Ph.D. Dissertation. Johns Hopkins University, Baltimore, Maryland, USA.

[58] Cameron Moy, Phúc C Nguyen, Sam Tobin-Hochstadt, and David Van Horn. 2020. Corpse Reviver: Sound and Efficient Gradual Typing via Contract Verification. arXiv preprint arXiv:2007.12630 (2020).

[59] James Noble, Andrew P Black, Kim B Bruce, Michael Homer, and Timothy Jones. 2017. Grace's Inheritance. Journal of Object Technology 16, 2 (2017).

[60] Nikolaos Papaspyrou and Konstantinos Sagonas. 2012. On Preserving Term Sharing in the Erlang Virtual Machine. In Proceedings of the Eleventh ACM SIGPLAN Workshop on Erlang Workshop (Copenhagen, Denmark) (Erlang '12). Association for Computing Machinery, New York, NY, USA, 11-20. https://doi.org/10.1145/2364489.2364493

[61] Michael Papathomas. 1989. Concurrency issues in object-oriented programming languages. Object Oriented Development (1989), 207245.

[62] Benjamin C. Pierce. 2002. Types and programming languages. MIT Press.

[63] Richard Roberts, Stefan Marr, Michael Homer, and James Noble. 2019. Transient Typechecks Are (Almost) Free. In 33rd European Conference 
on Object-Oriented Programming, ECOOP 2019, fuly 15-19, 2019, London, United Kingdom (LIPIcs, Vol. 134), Alastair F. Donaldson (Ed.). Schloss Dagstuhl - Leibniz-Zentrum für Informatik, 5:1-5:28. https://doi.org/10. 4230/LIPIcs.ECOOP.2019.5

[64] Venetia Laura Delano Robertson. 2013. Of ponies and men: My Little Pony: Friendship is Magic and the Brony fandom. International fournal of Cultural Studies (2013).

[65] Barry K. Rosen, Mark N. Wegman, and F. Kenneth Zadeck. 1988. Global Value Numbers and Redundant Computations. In Conference Record of the Fifteenth Annual ACM Symposium on Principles of Programming Languages, San Diego, California, USA, January 10-13, 1988, Jeanne Ferrante and P. Mager (Eds.). ACM Press, 12-27. https://doi.org/10.1145/ 73560.73562

[66] Stefan Savage, Michael Burrows, Greg Nelson, Patrick Sobalvarro, and Thomas E. Anderson. 1997. Eraser: A Dynamic Data Race Detector for Multithreaded Programs. ACM Trans. Comput. Syst. 15, 4 (1997), 391-411. https://doi.org/10.1145/265924.265927

[67] Jan Schäfer and Arnd Poetzsch-Heffter. 2010. JCoBox: Generalizing Active Objects to Concurrent Components, See [30], 275-299. https: //doi.org/10.1007/978-3-642-14107-2_13

[68] Ilya Sergey and Dave Clarke. 2012. Gradual Ownership Types. In Programming Languages and Systems - 21st European Symposium on Programming, ESOP 2012, Held as Part of the European foint Conferences on Theory and Practice of Software, ETAPS 2012, Tallinn, Estonia, March 24 April 1, 2012. Proceedings (Lecture Notes in Computer Science, Vol. 7211), Helmut Seidl (Ed.). Springer, 579-599. https://doi.org/10.1007/978-3-64228869-2_29

[69] Jeremy G. Siek and Walid Taha. 2006. Gradual Typing for Functional Languages. In IN SCHEME AND FUNCTIONAL PROGRAMMING WORKSHOP. 81-92.

[70] Jeremy G. Siek, Michael M. Vitousek, Matteo Cimini, and John Tang Boyland. 2015. Refined Criteria for Gradual Typing. In 1st Summit on Advances in Programming Languages, SNAPL 2015, May 3-6, 2015, Asilomar, California, USA (LIPIcs, Vol. 32), Thomas Ball, Rastislav Bodík, Shriram Krishnamurthi, Benjamin S. Lerner, and Greg Morrisett (Eds.) Schloss Dagstuhl - Leibniz-Zentrum fuer Informatik, 274-293. https: //doi.org/10.4230/LIPIcs.SNAPL.2015.274

[71] Tom Van Cutsem and Mark S. Miller. 2013. Trustworthy Proxies: Virtualizing Objects with Invariants. In Proceedings of the 27th European Conference on Object-Oriented Programming (Montpellier, France) (ECOOP'13). Springer-Verlag, Berlin, Heidelberg, 154-178. https://doi.org/10.1007/978-3-642-39038-8_7

[72] Edwin M. Westbrook, Jisheng Zhao, Zoran Budimlic, and Vivek Sarkar 2012. Practical Permissions for Race-Free Parallelism. In ECOOP 2012 - Object-Oriented Programming - 26th European Conference, Beijing, China, Fune 11-16, 2012. Proceedings (Lecture Notes in Computer Science, Vol. 7313), James Noble (Ed.). Springer, 614-639. https://doi.org/10.1007/ 978-3-642-31057-7 27

[73] Christian Wimmer and Thomas Würthinger. 2012. Truffle: a selfoptimizing runtime system. In Conference on Systems, Programming, and Applications: Software for Humanity, SPLASH '12, Tucson, AZ, USA, October 21-25, 2012, Gary T. Leavens (Ed.). ACM, 13-14. https://doi.org/ 10.1145/2384716.2384723

[74] Andrew K. Wright and Matthias Felleisen. 1994. A Syntactic Approach to Type Soundness. Inf. Comput. 115, 1 (1994), 38-94. https://doi.org/10. 1006/inco.1994.1093

[75] Tobias Wrigstad, Filip Pizlo, Fadi Meawad, Lei Zhao, and Jan Vitek. 2009. Loci: Simple Thread-Locality for Java. In ECOOP 2009 - ObjectOriented Programming, 23rd European Conference, Genoa, Italy, Fuly 6-10, 2009. Proceedings (Lecture Notes in Computer Science, Vol. 5653), Sophia Drossopoulou (Ed.). Springer, 445-469. https://doi.org/10.1007/ 978-3-642-03013-0_21

[76] Derek Wyatt. 2013. Akka concurrency. Artima Incorporation.
[77] Yang Zhao and John Boyland. 2008. A Fundamental Permission Interpretation for Ownership Types. In TASE. 65-72. 


\section{A Expression Rules Producing Errors}

Fig. 12 shows all the expression rules producing errors.

\section{B Definition, Lemmas, and Proofs}

Definition B.1 (Initial Configuration). An initial configuration is a closed term with empty heap, $\epsilon ; t$.

Definition B.2 (Terminal Configuration). A well-formed configuration $\Gamma \vdash H ; \bar{T}$ is terminal if it contains zero threads $(\bar{T}=\epsilon)$, it is an error $(\bar{T}=E r r)$, or if it is a deadlock configuration $(\operatorname{Deadlock}(\Gamma \vdash H ; \bar{T}))$.

Definition B.3 (Deadlock Configuration). A deadlocked configuration is a well-formed configuration where all threads are blocked on sends and receives.

Deadlock $(\Gamma \vdash H ; \bar{T}) \Longleftrightarrow$
$\bar{T} \neq \epsilon \wedge \forall T^{\prime} \in \bar{T} . \vee\left\{\begin{array}{l}T^{\prime}=E[\leftarrow \iota] \wedge H(\iota)=\operatorname{chan}\{, \varnothing\} \\ T^{\prime}=E\left[x \leftarrow \_\right] \wedge H(\iota)=\operatorname{chan}\{, v\} \\ T^{\prime}=E\left[\mathbf{\square}_{i} \iota\right] \wedge H(\iota)=\operatorname{chan}\left\{i_{-}\right\}\end{array}\right.$

Definition B.4 (Data Race). To formally define a data race, we introduce the notion of a trace $R$, which is an ordered sequence of reductions $r_{1}, r_{2} \ldots$ in the evaluation of a program $P$. We only concern ourselves with traces that do not evaluate to a permission or cast error.

We define the precedes relation $<$ on reductions thus: If $R=r_{1}, R^{\prime}$, then $r_{1}<r_{2}$ for all $r_{2} \in R^{\prime}$. Recall that threads have unique ids.

We are now ready to formally define a data race in Dalarna. A program has a data race if it may give rise to at least one (non-erring) trace with a data race. A trace $R$ has a data race if it contains steps $r_{1}<r_{2}$ such that

a) $r_{1}=H_{1} ; E\left[x . f={ }_{-}\right]^{i}$, and $H_{1}(x)=l($ thread $i$ writes $l . f)$

b) $r_{2}=H_{2} ; E\left[y \cdot f={ }_{-}\right]^{j}$ or $r_{2}=H_{2} ; E[y \cdot f]^{j}$, and $H_{2}(y)=$ $l$

(thread $j$ reads or writes $l . f$ )

c) $i \neq j$ (the conflicting accesses take place in different threads and there does not exist $r_{3}, r_{4} \in R$ such that

$$
\begin{aligned}
& \text { i } r_{1}<r_{3}<r_{4}<r_{2}, \\
& \text { ii } r_{3}=H_{3} ; E\left[-\leftarrow v_{1}\right]^{i} \text { and } l \in R O G\left(H, v_{1}\right) \text { (thread } i \text { sends } \\
& l \text { somewhere) } \\
&\text { iii } \left.r_{4}=H_{4} ; \leftarrow v_{2}\right]^{j} \text { and } l \in R O G\left(H, v_{2}\right) \text { (thread } j \text { receives } \\
& \quad l \text { from somewhere) }
\end{aligned}
$$

where $R O G(H, v)$ denotes the transitive closure of objects (the Reachable Object Graph) in $H$ rooted in $v$.

Definition B.5 (Trace). Define a trace $\mathcal{T}$ as a list of pairs containing the reduction rules and thread ids.

Definition B.6 (Trace Of A Program). Define the trace $\mathcal{T}$ of a program $P$ as the accumulation of pairs of reduction rules and thread ids on which the reduction rules take place, e.g., $\epsilon ; P \rightsquigarrow{ }^{*} H^{\prime} ; \overline{T^{\prime}}$ produces trace $\mathcal{T}$.
Definition B.7 (Trace Reduction Replay). Define a trace replay reduction relation $\left(\mathcal{T}: \mathcal{T}^{\prime}\right) ; H ; \bar{T} \leadsto \mathcal{T}^{\prime} ; H^{\prime} ; \overline{T^{\prime}}$ as the reduction relation that executes the first item of the trace $\mathcal{T}: \mathcal{T}^{\prime}$ at a time, returning the trace list without the first item $(\mathcal{T})$, a new heap $\left(H^{\prime}\right)$, and a new thread configuration $\left(\overline{T^{\prime}}\right)$.

Definition B.8 states that if an object is reachable from threads $t$ and $t^{\prime}$ then either $t=t^{\prime}$ or the object is not local or the object can only be dereference in a single thread (via ownership check). Thus, thread-local objects are not reachable by multiple threads. The helper function $\operatorname{ROG}(H, t)$ returns the set of objects in $H$ reachable from $t$ by traversing variables and fields (a reflexive transitive closure of objects).

Definition B.8 (Thread-Local Objects). A configuration satisfies object "thread-locality" if no object with a local capability is reachable from more than one thread.

$$
\begin{aligned}
\operatorname{Local}(H, \bar{T}) & \Longleftrightarrow \forall t^{i}, t^{\prime j} \in \bar{T} . \\
\iota & \in\left(\operatorname{ROG}\left(H, t^{i}\right) \cap \operatorname{ROG}\left(H, t^{\prime j}\right)\right) \Rightarrow \\
& \left(t=t^{\prime} \wedge i=j\right) \vee\left(H(\iota)=K \text { obj }\left\{_{--}\right\} \wedge K \neq \text { local }\right) \vee \\
& \left(t \neq t^{\prime} \wedge i \neq j \wedge(\operatorname{isLocal}(H, \iota)\right. \\
& \Rightarrow \text { isOwner }(H, i, \iota) \wedge \neg \text { isOwner }(H, j, \iota))
\end{aligned}
$$

Object isolation (Definition B.9) states that all isolated objects have a single reference to them, from the heap or stack, modulo borrowing. Borrowing allows calling methods on isolated objects without consuming the target, leading to (temporal) aliases which are all on the stack of the same thread. We forbid consuming self, thus aliases of isolated objects (and self) are only introduced through method calls. After a method call, the alias is buried and non-accessible.

Definition B.9 (Object Isolation). A configuration satisfies object isolation if no objects with an iso capability have more than one incoming pointer from the stack and heap, modulo borrowing.

$$
\begin{aligned}
& \text { Isolated }(H, \bar{T}) \Longleftrightarrow \\
& \quad \forall \iota \in \operatorname{Isos}(H) .|\operatorname{Inc}(H, \bar{T}, \iota) \cup \operatorname{Inc}(H, \iota)|>1 \Rightarrow \\
& \quad \exists t \in \bar{T} \cdot \operatorname{Inc}(H, \bar{T}, \iota)=\operatorname{Inc}(H, t, \iota) \wedge \operatorname{Inc}(H, \iota)=\emptyset
\end{aligned}
$$

where Inc collects the set of variables and fields aliasing a particular location: $\operatorname{Inc}(H, v)=\{\iota . f \mid \iota \in \operatorname{dom}(H) \wedge H(\iota . f)=$ $v\} \cup\{\iota \mid \iota \in \operatorname{dom}(H) \wedge H(\iota)=\operatorname{chan}\{i, v\}\}, \operatorname{Inc}(H, \bar{T}, v)=$ $\bigcup_{t \in \bar{T}} \operatorname{Inc}(H, t, v), \operatorname{Inc}(H, t, v)=\{x \mid x \in \operatorname{FreeVars}(t) \wedge H(x)=$ $v\} \cup\{v \mid v \in t\}$, and FreeVars $(t)$ is the set of all free variables in $t$.

Definition B.10 (Object's Owner). Define the predicate isOwner $(H, i, v)$ for a heap $(H)$, owner $(i)$, and value $(v)$ as:

$$
\begin{aligned}
\text { isOwner }(H, i, v) & \Longleftrightarrow H(\iota)=K^{i} \operatorname{obj}\left\{_{--}\right\}, \\
& \text {where } v=\iota \wedge \iota \in \operatorname{dom}(H)
\end{aligned}
$$




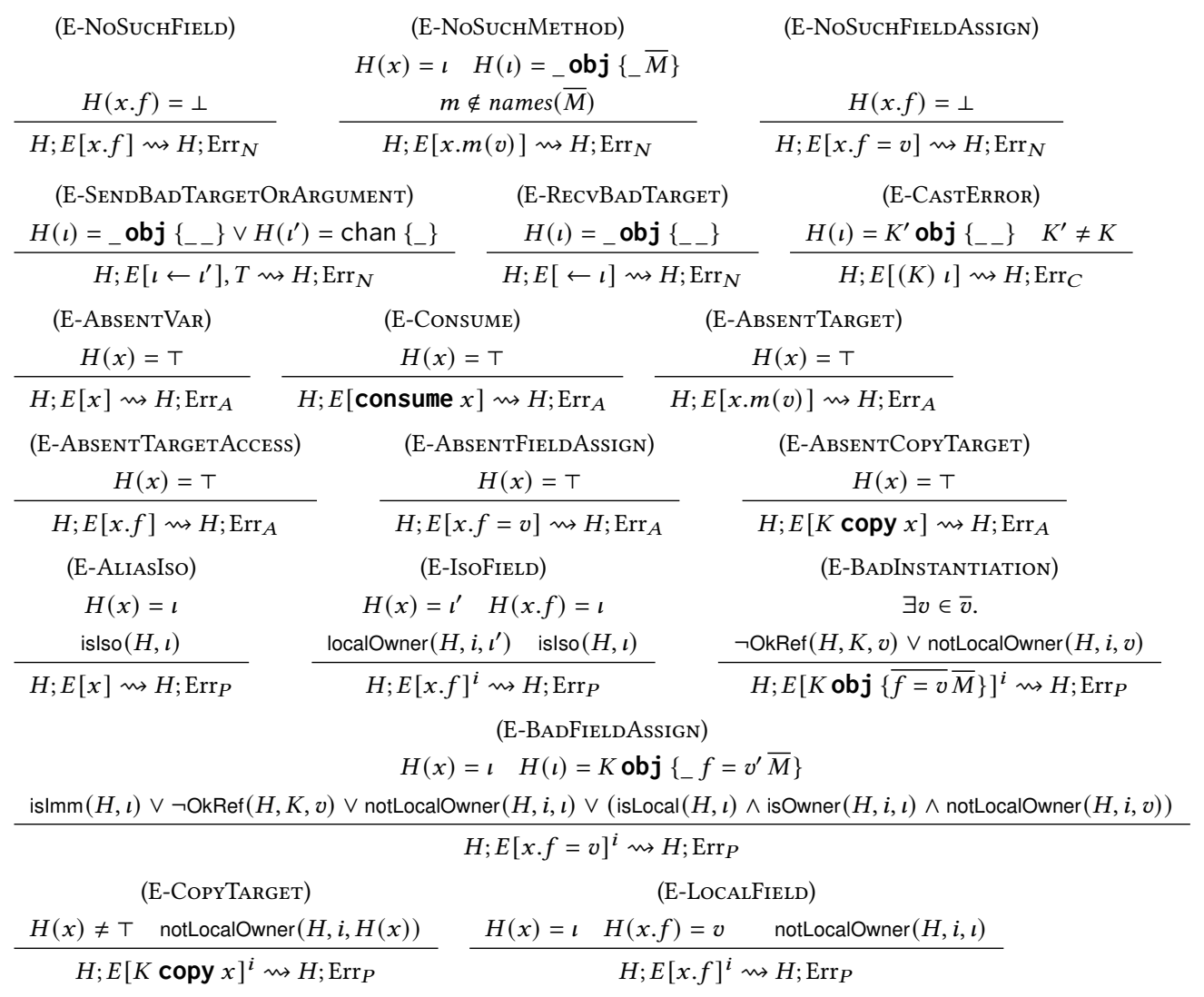

Figure 12. Expression rules producing errors. To reduce clutter, we write $H(x . f)=v$ when $H(x)=\iota \wedge H(\iota)={ }_{-}$obj $\left\{F_{-}\right\}$ and $f=v \in F$, and $H(x . f)=\perp$ when $H(x)=\iota \wedge H(\iota)={ }_{-}$obj $\left\{F_{-}\right\}$and $f \notin \operatorname{dom}(F)$, and notLocalOwner $(H, i, v)=$ isLocal $(H, v) \wedge \neg$ isOwner $(H, i, v)$.

Definition B.11 (Safe Erasure). Define the function safe erasure, ${ }^{e}$, as the function that replaces safe capabilities to unsafe capability as follows:

$$
\begin{aligned}
& (\Gamma, x: K)^{e}=(\Gamma)^{e}, x: \text { unsafe } \\
& (\Gamma, \iota: K)^{e}=(\Gamma)^{e}, \iota: \text { unsafe } \\
& (H, x \mapsto \iota)^{e}=(H)^{e}, x \mapsto \iota \\
& (H, \iota \mapsto \operatorname{chan}\{i, v\})^{e}=(H)^{e}, \iota \mapsto \operatorname{chan}\{i, v\} \\
& \left(H, \iota \mapsto K \text { obj }\left\{\_-\right\}\right)^{e}=(H)^{e}, \iota \mapsto \text { unsafe obj }\left\{\_-\right\} \\
& (t \bar{T})^{e}=(t)^{e}(\bar{T})^{e} \\
& (t)^{e}= \begin{cases}\text { unsafe obj }\left\{\overline{f=(v)^{e}} \overline{(M)^{e}}\right\}, & t=K \text { obj }\{\overline{f=v} \bar{M}\} \\
(\text { unsafe }) \iota, & t=(K) \iota \\
\text { unsafe copy } x, & t=K \operatorname{copy} x \\
\text { let } x=(e)^{e} \text { in }(t)^{e}, & t=\text { let } x=e \text { in } t \\
\text { spawn }(x)\left\{(t)^{e}\right\}, & t=\operatorname{spawn}(x)\{t\} \\
\text { method } m(x)\left\{(t)^{e}\right\}, & t=\operatorname{method} m(x)\{t\} \\
t, & \text { otherwise }\end{cases}
\end{aligned}
$$

Definition B.12 (Capability Extraction).

$$
\operatorname{Cap}(H, x)= \begin{cases}K, & H(x)=\iota \wedge H(\iota)=K \text { obj }\left\{_{--}\right\} \\ \text {local, } & H(x)=\iota \wedge H(\iota)=\operatorname{chan}\left\{\left\{_{-}\right\}\right. \\ K, & H(x)=\top, \text { for some } K\end{cases}
$$

Definition B.13 (Thread's Variables). The variables of a thread are all variables used in the thread.

$$
\begin{aligned}
& \operatorname{Vars}(\text { let } x=e \text { in } t)=\{x\} \cup \operatorname{Vars}(e) \cup \operatorname{Vars}(t) \\
& \operatorname{Vars}(x)=\operatorname{Vars}(\operatorname{consume} x)=\operatorname{Vars}(x \cdot f)=\operatorname{Vars}(K \operatorname{copy} x)=\{x\} \\
& \operatorname{Vars}(x \cdot f=e)=\operatorname{Vars}(x \cdot m(e))=\{x\} \cup \operatorname{Vars}(e) \\
& \operatorname{Vars}\left(e \leftarrow e^{\prime}\right)=\operatorname{Vars}(e) \cup \operatorname{Vars}\left(e^{\prime}\right) \\
& \operatorname{Vars}((K) e)=\operatorname{Vars}(\leftarrow e)=\operatorname{Vars}(e) \\
& \operatorname{Vars}\left(K \text { obj }\left\{\overline{f=w_{-}}\right\}\right)=\operatorname{Vars}(\bar{w}) \\
& \operatorname{Vars}(\operatorname{spawn}(x)\{t\})=\operatorname{Vars}\left({ }_{-}\right)=\epsilon
\end{aligned}
$$

Definition B.14 (Reachable Object Graph). Define the helper function $\operatorname{ROG}(H, t)$ and $\operatorname{ROG}(H, \iota)$ as the function that returns the transitive closure of objects reachable from term $t$ 
and location $\iota$.

$$
\begin{aligned}
& \operatorname{ROG}(H, t)=\bigcup_{x \in \operatorname{DefVar}(H, t)} \operatorname{ROG}(H, H(x)) \cup\{\operatorname{ROG}(H, \iota) \mid \iota \in t\} \\
& \operatorname{ROG}\left(H, v^{\prime}\right)= \begin{cases}\bigcup_{f=v \in \bar{F}} \operatorname{ROG}(H, v) \cup\{l\}, & v^{\prime}=\iota \wedge H(\iota)=K \operatorname{obj}\{\bar{F} \bar{M}\} \\
\emptyset, & \text { otherwise }\end{cases} \\
& \operatorname{Def} \operatorname{Var}(H, t)=\{x \mid x \in t \wedge x \in \operatorname{dom}(H)\}
\end{aligned}
$$

Lemma B.15 (Well-formed Values Are Not Absent (Or Null)).

$$
\Gamma \vdash H ; E[v] \Rightarrow v \neq \varnothing \wedge v \neq \top
$$

Proof. By induction on the well-formedness of the configuration.

Lemma B.16 (Fresh Variables).

$$
\begin{aligned}
& \Gamma \vdash H ; \text { let } x=e \text { in } t \Rightarrow x \notin \operatorname{dom}(\Gamma) \wedge x \notin \operatorname{dom}(H) \\
& \Gamma \vdash H ; \operatorname{spawn}(x)\{t\} \Rightarrow x \notin \operatorname{dom}(\Gamma) \wedge x \notin \operatorname{dom}(H)
\end{aligned}
$$

Explanation. When a term or expression introduces a new variable, the variable is new to $\Gamma$ and $H$.

Proof. Immediate by definition of well-formed configuration.

Lemma B.17 (Store Contains Top-Level Variables).

$\Gamma \vdash H$; let $x=e$ in $t \Rightarrow \operatorname{FreeVars}(e) \cup \operatorname{FreeLoc}(e) \subseteq \operatorname{dom}(H)$

Explanation. Given a thread $T=$ let $x=e$ in $t$, the free variables and locations of the expression e are in the domain of the store.

Proof. Immediate from definition of well-formed configuration.

Lemma B.18 (Weakened Well-formed Environment).

$$
\Gamma \vdash H \wedge x \notin \operatorname{dom}(\Gamma) \Rightarrow \Gamma, x: K \vdash H
$$

Explanation. If the store is a well-formed w.r.t. an environment, then extending the environment cannot contradict this fact.

Proof. Straightforward induction on the well-formedness rules.

Lemma B.19 (Typing Permutation).

$$
\Gamma \vdash t \wedge \Delta \text { is a permutation of } \Gamma \Rightarrow \Delta \vdash t
$$

Proof. By induction on the typing derivation $\Gamma \vdash t$.

Lemma B.20 (Weakening Typing Environment).

$$
\begin{aligned}
& \Gamma \vdash t \wedge x \notin \operatorname{dom}(\Gamma) \wedge x \notin \operatorname{Vars}(t) \Rightarrow \Gamma, x: K \vdash t \\
& \Gamma \vdash t \wedge \iota \notin \operatorname{dom}(\Gamma) \Rightarrow \Gamma, \iota: K \vdash t \\
& \Gamma \vdash e \wedge x \notin \Gamma \Rightarrow \Gamma, x: K \vdash e
\end{aligned}
$$

Proof. By induction over the term well-formedness. There are multiple cases to handle. We show the case when $t=$ let $y=e$ in $t^{\prime}$ which is the more interesting one. Case $t=$ let $y=e$ in $t^{\prime}$. By the initial assumptions:

$$
\begin{aligned}
& \Gamma \vdash \operatorname{let} y=e \text { in } t^{\prime} \\
& x \notin \operatorname{dom}(\Gamma) \\
& x \notin \operatorname{Vars}(t)
\end{aligned}
$$

\section{Need to show}

$$
\Gamma, x: K \vdash \text { let } y=e \text { in } t^{\prime}
$$

We prove each of the components of rule WF-LET.

1. By the initial assumption $\Gamma \vdash$ let $y=e$ in $t^{\prime}(4)$ and WF-LET, $y \notin \operatorname{dom}(\Gamma)$.

2. By 1 and 6 , we can conclude that $y \notin \operatorname{dom}(\Gamma, x: K)$.

3. For any $e$, we must prove that $\Gamma, x: K \vdash e$ is wellformed. We apply the induction hypothesis (Eq. (3)), and conclude that $\Gamma, x: K \vdash e$ is well-formed

4. We need to show that $\Gamma, x: K, y: K^{\prime} \vdash t^{\prime}$. By the initial hypothesis $\Gamma \vdash$ let $y=e$ in $t^{\prime}(4)$. As part of the assumptions $\Gamma, y: K^{\prime} \vdash t^{\prime}$ holds. By the induction hypothesis Eq. (1), we conclude that $\Gamma, x: K, y: K^{\prime} \vdash t^{\prime}$.

5. From 3 and 4 and by typing rule WF-LET, we can conclude $\Gamma, x: K \vdash$ let $y=e$ in $t^{\prime}$.

The proof of Eqs. (2) and (3) follow the same proof strategy.

Lemma B.21 (Weakening).

$$
\begin{gathered}
\Gamma \vdash H ; t \wedge \Gamma^{\prime}=\Gamma, \Gamma^{\prime \prime} \wedge\left(\operatorname{dom}\left(\Gamma^{\prime \prime}\right) \cap \operatorname{dom}(\Gamma) \cap \operatorname{Vars}(t)=\epsilon\right) \wedge H^{\prime} \supseteq H \\
\wedge \Gamma^{\prime} \vdash H^{\prime} \Rightarrow \Gamma^{\prime} \vdash H^{\prime} ; t
\end{gathered}
$$

Proof. By induction on the shape of $t$. The most interesting case is $t=$ let $x=e$ in $t^{\prime}$ and the others follow the same proof technique. We need to prove:

$$
\begin{gathered}
\Gamma \vdash H ; \text { let } x=e \text { in } t^{\prime} \wedge \Gamma^{\prime}=\Gamma \cup \Gamma^{\prime} \wedge \Gamma^{\prime} \supseteq \Gamma \wedge H^{\prime} \supseteq H \\
\wedge \Gamma^{\prime \prime} \cap \Gamma \cap \operatorname{Vars}\left(\text { let } x=e \text { in } t^{\prime}\right)=\epsilon \wedge \Gamma^{\prime} \vdash H^{\prime} \\
\Rightarrow \Gamma^{\prime} \vdash H^{\prime} ; \text { let } x=e \text { in } t^{\prime}
\end{gathered}
$$

We prove that if all components are well-formed, then by rule WF-Term $\Gamma^{\prime} \vdash H^{\prime}$; let $x=e$ in $t^{\prime}$.

1. From the assumptions, $\Gamma^{\prime}+H^{\prime}$.

2. We want to prove that $\Gamma^{\prime}+$ let $x=e$ in $t^{\prime}$. From the initial assumptions, $\Gamma \vdash$ let $x=e$ in $t^{\prime}$ and $x \notin \operatorname{dom}(\Gamma)$ and $x \notin \operatorname{Vars}(t)$ and $\Gamma^{\prime} \supseteq \Gamma$. By multiple applications of Lemma B.20, we conclude $\Gamma^{\prime} \vdash$ let $x=e$ in $t^{\prime}$.

3. From 1 and 2, and by application of WF-Term, conclude $\Gamma^{\prime} \vdash H^{\prime} ;$ let $x=e$ in $t^{\prime}$. 
Lemma B.22 (Weakening Maintains Thread Locality).

$$
\begin{aligned}
\bar{T} & \equiv t \overline{T^{\prime}} \wedge \operatorname{Local}(H, \bar{T}) \wedge \iota \in \operatorname{ROG}(H, t) \\
& \wedge \operatorname{isLocal}(H, \iota) \wedge x \notin \operatorname{dom}(H) \Rightarrow \operatorname{Local}\left((H, x \mapsto \iota), t \overline{T^{\prime}}\right)
\end{aligned}
$$

Explanation. Extending the heap with new variables does not affect thread-local objects.

Proof. Trivially satisfied by induction on the definition of $\operatorname{Local}(H, \bar{T})$.

Lemma B.23 (Binding Isolate Locations Maintain Isolatedness).

$\bar{T} \equiv t \overline{T^{\prime}} \wedge \operatorname{Isolated}(H, \bar{T}) \wedge \iota \in t \wedge \operatorname{islso}(H, \iota) \Rightarrow \operatorname{Isolated}((H, x \mapsto$

Explanation. Extending the heap with a stack variable over an isolated location maintains isolation.

Proof. Trivially satisfied by induction on the definition of Isolated $(H, \bar{T})$.

Lemma B.24 (Thread-Local Term Substitution Maintains Locatity).

$$
\begin{aligned}
t^{\prime} \subseteq t & \wedge \operatorname{Local}(H, t \bar{T}) \wedge \iota \in \operatorname{ROG}(H, t) \wedge \operatorname{isLocal}(H, \iota) \\
& \wedge x \notin \operatorname{dom}(H) \wedge \operatorname{FreeVar}\left(t^{\prime}\right) \subseteq \operatorname{FreeVar}(t) \cup\{x\} \\
& \Rightarrow \operatorname{Local}\left((H, x \mapsto \iota), t^{\prime} \bar{T}\right)
\end{aligned}
$$

Explanation. If an object $\iota$ is thread-local to term $t$, there is a subterm $t^{\prime}$ of $t$, and we extend the heap to map a stack variable to $\iota$, then replacement of $t$ by $t^{\prime}$ maintains thread-locality under the modified heap.

Proof. By induction on the shape of $t^{\prime} \cdot t^{\prime}$ can only use free variables available in $t$ and the other threads cannot point to the introduced variable $x$ as $x \notin \operatorname{dom}(H)$. Hence, threadlocality is maintained for object in $\iota$.

Lemma B.25 (Deterministic Evaluation). If $H ; T \bar{T} \leadsto H^{\prime} ; \overline{T^{\prime}} \bar{T}$ and $H ; T \bar{T} \leadsto H^{\prime \prime} ; \overline{T^{\prime \prime}} \bar{T}$, then $\overline{T^{\prime}} \equiv \overline{T^{\prime \prime}}$

Proof. By induction on a derivation of $H ; T \bar{T} \leadsto H^{\prime} ; \overline{T^{\prime}} \bar{T}$.

Lemma B.26 (Capability Stripping Preserves Terms and Expressions). $\left(t^{i}\right)^{e}=t^{i}[\mathrm{~K} /$ unsafe] Explanation: A capability stripped term is equivalent to a replacement of its capabilities $K$ by unsafe, i.e., preserves the terms and expressions.

Proof. By induction on the term structure.

Corollary 5.3 (Thread-Affinity of Thread-Local Fields). Implied by Preservation, a thread local object with owner $i$ cannot contain a thread local object with owner $j$, where $i \neq j$. The only way a local object can reference another local object of a different owner is via field assignment (R-FIELDASSIGN). But RFIELDASSIGN checks that target and source share owners. Thus, thread local objects can only reference thread local objects of the same owner.
Proof. By induction on the thread structure. Assume we are deadling with thread local objects. The only way to create a thread local object with a reference to a thread local object with different owner is by rule R-FIELDUPDATE. But this rule does not satisfy the premises when the owner of the target and source objects have different owners. Instead, rule EBADFIELDASSIGN applies and throws a permission error.

Lemma B.27 (Unsafe Programs Do Not Raise Permission Errors). If $\Gamma^{e} \vdash(H ; \bar{T})^{e}$ and $(H ; \bar{T})^{e} \leadsto H^{\prime} ; \overline{T^{\prime}}$, then $\operatorname{Err}_{C} \not \equiv$ $\overline{T^{\prime}} \not \equiv \operatorname{Err}_{P}$.

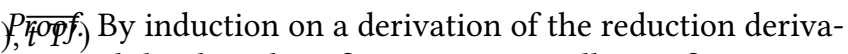
tion and the thread configuration. Trivially, configurations that do not throw runtime errors satisfy the inductive hypothesis; configurations where $T^{\prime} \equiv \operatorname{Err}_{N}$ or $T^{\prime} \equiv \operatorname{Err}_{C}$ trivially satisfy the hypothesis; from the reduction rules that throw permission errors: E-AliasIso, E-IsoField, EBadFieldAssign, E-CopyTarget, E-BadInstantiation and E-LOCALFIELD require safe capabilities to throw a permission error but by the initial assumptions, we have stripped the safe capabilities. Thus, these errors cannot happen. Reduction rule E-BADINSTANTIATION cannot happen because all capabilities are unsafe and $\operatorname{OkRef}(. .$.$) is trivially satisfied.$

Theorem 5.1 (Progress). A well-formed configuration $\Gamma \vdash$ $H ; \bar{T}$ is either a terminal configuration or $H ; \bar{T} \leadsto H^{\prime} ; \overline{T^{\prime}}$.

Proof. Assumptions $\Gamma \vdash H ; \bar{T}$.

We start by induction on the shape of $\bar{T}$.

1. $\bar{T} \equiv$ Err. Progress holds trivially.

2. $\bar{T} \equiv \epsilon$. Progress holds trivially.

3. $\bar{T} \equiv T \overline{T^{\prime}}$. Induction hypothesis:

IH1 $T=\operatorname{Err} \vee \operatorname{Deadlock}(\Gamma \vdash H ; T) \vee T=\epsilon \vee H ; T \rightsquigarrow$ $H^{\prime} ; \overline{T^{\prime \prime}}$

IH2 $\overline{T^{\prime}}=\operatorname{Err} \vee \operatorname{Deadlock}\left(\Gamma \vdash H ; \overline{T^{\prime}}\right) \vee \overline{T^{\prime}}=\epsilon \vee H ; \overline{T^{\prime}} \leadsto$ $H^{\prime} ; \overline{T^{\prime \prime}}$

IH3 Deadlock $\left(\Gamma \vdash H ; T \overline{T^{\prime}}\right)$

We continue by case analysis over IH1.

a. $T \equiv \operatorname{Err} \overline{T^{\prime}}$. By the equivalence rules, $T \equiv E r r$ and is a terminal configuration.

b. $T \equiv \epsilon$, then we continue by $\mathbf{I H} 2$.

c. $H ; T \leadsto H^{\prime} ; \overline{T^{\prime \prime}}$ by C-Eval.

d. Deadlock $(\Gamma \vdash H ; T)$. We continue by case analysis over IH2:

i. $\overline{T^{\prime}} \equiv E r r$. By the equivalence rules, T Err $\equiv$ Err and is a terminal configuration.

ii. $\overline{T^{\prime}}=\epsilon$. Then $\Gamma \vdash H ; T$ is a terminal configuration. iii. $H ; \overline{T^{\prime}} \leadsto H^{\prime} ; \overline{T^{\prime \prime}}$, the configuration steps to $H ; T \overline{T^{\prime}} \leadsto$ $H^{\prime} ; T \overline{T^{\prime \prime}}$ by rule C-Eval.

iv. Deadlock $\left(\Gamma \vdash H ; \overline{T^{\prime}}\right)$, then we proceed by IH3, Deadlock $\left(\Gamma \vdash H ; T \overline{T^{\prime}}\right)$, and progress holds trivially.

4. $\bar{T} \equiv t$. By the induction hypothesis either $\Gamma \vdash H$; $t$ is a terminal configuration or it can make progress. 
A1 Deadlock $(\Gamma \vdash H ; t) \vee H ; t \rightsquigarrow H^{\prime} ; \overline{T^{\prime}}$

We continue by case analysis over $\mathbf{A} \mathbf{1}$.

- Deadlock $(\Gamma \vdash H ; t)$. Progress holds trivially.

- $H ; t \rightsquigarrow H^{\prime} ; \overline{T^{\prime}}$.

From the initial assumption and WF-ConfIGURATION:

$$
\begin{aligned}
& \Gamma \vdash H \\
& \Gamma \vdash H ; t \\
& \forall t^{\prime} \in \bar{T} . \Gamma \vdash H ; t^{\prime}
\end{aligned}
$$

We proceed by induction over the shape of $t$, and assume that all subexpressions were reduced using the evaluation context $E$ rules. We prove all the inductive cases where the evaluation context reduces an expression to a value.

a. $t=\varnothing$. This cannot happen, follows from Lemma B.15 with Eq. (8).

b. $t \equiv$ let $x=v$ in $t^{\prime}$.

i. $x \notin \operatorname{dom}(H)$ from Lemma B.16

ii. By R-LET with (4(b)i) the configuration steps to $H, x \mapsto v ; t^{\prime}$.

c. $t \equiv v$. By the equivalence rules (§ 5.1), $H ; v \equiv$

$H ; \epsilon$ and we apply the induction hypothesis to this configuration.

d. $t \equiv E[x]$.

i. $x \in \operatorname{dom}(H)$ by Lemma B.17.

ii. $x \mapsto v \in H$ by assumption Eq. (7) with Item 4(d)i.

iii. From Item 4(d)ii we proceed by case analysis on the shape of $v$ :

- $H(x)=\mathrm{T}$. By E-ABSENTVAR the configuration steps to $H ; \operatorname{Err}_{A}$.

- $H(x)=\varnothing$. This cannot happen, follows from Lemma B.15 with Eq. (8)..

- $H(x)=\iota . \iota \in \operatorname{dom}(H)$ by assumption Eq. (7). There are two cases to consider, whether the location is to an isolated object or not.

* ᄀislso $(H, \iota)$. By R-VAR the configuration steps to $H ; E[\imath]$.

* islso $(H, \iota)$. By E-AliasIso the configuration steps to $H$; $\operatorname{Err}_{P}$.

e. $t \equiv E[$ consume $x]$.

i. $x \in \operatorname{dom}(H)$ by Lemma B.17.

ii. $x \mapsto v \in H$ by assumption Eq. (7) with Item 4(e)i.

iii. From Item 4(e)ii we proceed by case analysis on

the shape of $v$ :

$-v=\mathrm{T}$. By E-Consume the configuration steps to $H ; \operatorname{Err}_{A}$.

$-v=\varnothing$. This cannot happen, follows from Lemma B.15 with Eq. (8)..

$-v=\imath . \iota \in \operatorname{dom}(H)$ by assumption Eq. (7), and $H ; E[$ consume $x] \leadsto H[x \mapsto \mathrm{T}] ; E[\iota]$ by RConsume.

f. $t \equiv E[(K) t]$.

i. $\iota \in \operatorname{dom}(H)$ by Lemma B.17 ii. We proceed by case analysis on the object capability of the location $\iota$, checking whether the object capability matches the casted capability.

- $H(\iota)=K$ obj $\left\{{ }_{--}\right\}$. By R-CAstLoc the configuration steps to $H ; E[l]$.

- $H(\iota)=K^{\prime}$ obj $\left\{{ }_{--}\right\} \wedge K \neq K^{\prime}$. By E-CASTERror the configuration steps to $H$; $\operatorname{Err}_{A}$.

g. $t \equiv E[x . f]$. There are multiple cases to handle, depending on the value of $x$ and its field $f$.

i. $x \in \operatorname{dom}(H)$ by Lemma B.17.

ii. $x \mapsto v \in H$ by assumption Eq. (7) with Item 4(g)i.

iii. $v=\top \vee \iota \vee \varnothing$. We proceed by case analysis over

$v$ :

$-v=\varnothing$. This cannot happen. By assumption

(7), $x \mapsto \varnothing \in H$ is not well-formed.

$-v=\mathrm{T}$. By E-AbsentTARget Access the configuration steps to $H ; \operatorname{Err}_{A}$.

$-v=\iota$. By the assumption Eq. (7), $\iota \mapsto{ }_{-}$obj $\left\{{ }_{-}\right\} \in$ $H$. We proceed by case analysis on the field.

* $H(\iota)={ }_{\text {obj }}\{\bar{F} \bar{M}\}, f \notin \operatorname{dom}(\bar{F})$. By E-NoSuchField the configuration steps to $H$; $\operatorname{Err}_{N}$.

* $H(\iota)={ }_{-}$obj $\left\{\_f=v \bar{M}\right\}$, $\neg$ islso $(H, \iota)$, localOwner $(H, i, \iota)$. By R-FIELD the configuration steps to $H$; v

* $H(\iota)={ }_{-}$obj $\left\{\_f=v \bar{M}\right\}$, islso $(H, \iota)$, localOwner $(H, i, \iota)$. By E-IsoField the configuration steps to $H$; $\operatorname{Err}_{P}$.

* $H(\iota)={ }_{-}$obj $\left\{\_f=v \bar{M}\right\}$, noLocalOwner $(H, i, \iota)$. By E-LocalField the configuration steps to $H$; $\operatorname{Err}_{P \text {.. }}$

h. $t \equiv E[x . f=v]$ There are multiple cases to handle, depending on the value of $x$ and its field $f$.

i. $x \in \operatorname{dom}(H)$ by Lemma B.17.

ii. $x \mapsto v^{\prime} \in H$ by assumption Eq. (7) with Item 4(h)i.

iii. $v=T \vee \iota \vee \varnothing$. We proceed by case analysis over $v^{\prime}$ and $v$ :

$-v^{\prime}=\varnothing$. This cannot happen. By assumption (7), $x \mapsto \varnothing \in H$ is not well-formed.

$-v^{\prime}=\mathrm{T}$. By E-AbSEntFieldAssign the configuration steps to $H$; $\operatorname{Err}_{A}$..

- $v^{\prime}=\iota^{\prime}$. By the assumption Eq. (7), $\iota \mapsto{ }_{-}$obj $\left\{{ }_{--}\right\} \in$ $H$. There are multiple cases to handle. We start by case analysis on the value of $v$, and then continue by case analysis on field $f$ :

$* v=\varnothing$. This cannot happen, follows from Lemma B.15 with Eq. (8).

$* v=\mathrm{T}$. This cannot happen, by Lemma B.15 with Eq. (8)..

$* v=\imath$. We proceed by case analysis over field $f$ below:

* $H\left(\iota^{\prime}\right)={ }_{-}$obj $\{\bar{F} \bar{M}\}, f \notin \operatorname{dom}(\bar{F})$. By ENoSuchFieldAssign the configuration steps to $H ; \operatorname{Err}_{N}$. 
* $H\left(\iota^{\prime}\right)=K$ obj $\left\{\_f=v \bar{M}\right\} \wedge K=$ imm. By E-BADFIELDAssign the configuration steps to $H$; $\operatorname{Err}_{P}$.

* $H(\iota)=K$ obj $\left\{\_f=v^{\prime \prime} \bar{M}\right\} \wedge K \neq \mathrm{imm}, v^{\prime \prime}=$ $\iota^{\prime \prime}$. If the object location $\iota^{\prime \prime}$ has a capability $K^{\prime}$, s.t. $K, K^{\prime} \leq$ and the source and target are owned by the current thread, then we conclude by direct application of R-FIELDAssign. If the object location $\iota^{\prime \prime}$ has a capability $K^{\prime}$ s.t. $K \not K^{\prime}$ or if the current thread does not own the target or if the current thread is the owner of the target but not of the source, then we conclude by direct application of $\mathrm{E}$ BADFIELdAssign, which reduces to $H$; $\operatorname{Err}_{P}$. i. $t \equiv E\left[v \leftarrow v^{\prime}\right]$ Reminder. We are under the assumption that $\neg \operatorname{Deadlock}\left(\Gamma \vdash H ; t \overline{T^{\prime}}\right)$, so $t$ may be blocked but there is no deadlock in the global configuration $\left(\overline{T^{\prime}}\right)$. We proceed by case analysis on the shape of $v$ and $v^{\prime}$.

i. $v=\varnothing \vee v^{\prime}=\varnothing$. This cannot happen, follows from Lemma B.15 with Eq. (8)..

ii. $v=\mathrm{T} \vee v^{\prime}=\mathrm{T}$. This cannot happen, follows from Lemma B.15 with Eq. (8)..

iii. $v=\iota \wedge v^{\prime}=\iota^{\prime}$. There are multiple cases to handle, depending on the shape of the objects pointed by $\iota$ and $\iota^{\prime}$.

- $H(\iota)==_{-}$obj $\left\{{ }_{-}\right\}$. By E-SendBAdTARgetOrArgument the configuration steps to $H ; \operatorname{Err}_{N}$.

- $H\left(\iota^{\prime}\right)=$ chan $\left\{{ }_{-}\right\}$. By E-SendBAdTARgEtORARgument the configuration steps to $H$; $\operatorname{Err}_{N}$.

- $H(\iota)=$ chan $\{, \varnothing\}$, by R-SENDBLOCK the configuration steps.

j. $t \equiv E[\leftarrow \iota]$. By Lemma B.17, $\iota \in \operatorname{dom}(H)$. There are two cases to handle, when $\iota$ maps to an object and to a channel.

- $\iota \mapsto \_$obj $\left\{{ }_{-}\right\}$. By E-RecvBadTARGet the configuration steps to $H$; $\operatorname{Err}_{N}$.

- $\iota \mapsto \operatorname{chan}\{, v\} . v=\iota^{\prime}$ by assumption (7) and the assumption that it takes a reduction step (the deadlock was handled before) $\iota \mapsto$ chan $\left\{\iota_{-} \iota^{\prime}\right\}$. By R-Recv the configuration steps to $H[\iota \mapsto$ chan $\{, \varnothing\}] ; E\left[\iota^{\prime}\right]$.

k. $t \equiv E[x \cdot m(v)]$

i. $x \in \operatorname{dom}(H)$ by Lemma B.17.

ii. Given $(4(\mathrm{k}) \mathrm{i})$, we do case analysis on the shape of the value pointed by $x$ in the store.

- $x \mapsto \mathrm{T}$. By E-ABSENTTARgET the configuration steps to $H ; \operatorname{Err}_{A}$.

$-x \mapsto \iota$. By the assumptions of WF-Configuration, $\iota \in \operatorname{dom}(H)$ (with WF-H-VAR). We proceed by case analysis on whether $m$ exists in $\bar{M}$ :

* $m \notin$ names $(\bar{M})$. we conclude with E-NoSuchMethod and $H ; \operatorname{Err}_{N}$.
* $m \in \operatorname{names}(\bar{M})$. we conclude by direct application of R-CALL, $H, x^{\prime} \mapsto \iota, y^{\prime} \mapsto v$; $E[t[$ self $=$ $\left.\left.x^{\prime}\right]\left[y=y^{\prime}\right]\right]$, where $x^{\prime}, y^{\prime}$ are fresh variables and $t$ is the method body of $m$.

l. $t \equiv E[K \operatorname{copy} x]$.

i. $x \in \operatorname{dom}(H)$, by Lemma B.17.

ii. By assumption (7), $x \mapsto v$ and $v=\top$ or $v=\iota$ and $\iota \in \operatorname{dom}(H)$. We proceed by case analysis on $v$ :

- $x \mapsto \top \in H$. By E-AbsentCopyTarget the configuration steps to $H$; $\operatorname{Err}_{A}$.

$-x \mapsto \iota \in H \wedge$ notLocalOwner $(H, i, l)$. By ECopyTARget the configuration steps to $H$; $\operatorname{Err}_{P}$.

- $x \mapsto \iota \in H \wedge$ localOwner $(H, i, l)$ By WF-Copy, iso $\neq K$. By R-Copy the configuration steps to $H^{\prime} ; E[\iota]$. where $\operatorname{OkDup}(H, K, \iota)=\left(H^{\prime}, \iota^{\prime}\right)$ where $\iota^{\prime}$ is the fresh location to the duplicated object graph and $H^{\prime}$ is the new store containing the duplicated object graph.

m. $t \equiv E\left[\right.$ spawn $\left.(x)\left\{t^{\prime}\right\}\right]$. By well-formed configuration (WF-Configuration) with WF-Spawn, $x \notin$ $\operatorname{dom}(\Gamma)$ and FreeVar $\left(t^{\prime}\right) \subseteq\{x\}$. By Lemma B.16, $x \notin \operatorname{dom}(H)$ either. We conclude by direct application of R-Spawn, $H, x \mapsto \iota, \iota \mapsto \operatorname{chan}\{i, \varnothing\} ; E[\iota] t$ where $t, i$ are fresh, and $t$ is a new spawned thread.

n. $t \equiv E\left[\mathbf{\square}_{i} \iota\right]$. By Lemma B.17, $\iota$ in $\operatorname{dom}(H)$. By wellformed configuration (WF-CONFIGURATION), we can further assume $\iota \mapsto$ chan $\left\{i^{\prime}, v\right\}$ (WF-Term). We proceed by case analysis on the index $i$ and value $v$.

- $i=i^{\prime}, v=\varnothing$, then by R-SendUnBlock we conclude with $H ; E[\iota]$.

$-i=i^{\prime}, v \neq \varnothing$, then the configuration is blocked. By the assumption of well-formed configuration WF-Configuration there is a $t^{\prime} \in \bar{T}$ that such that it is not deadlocked. We conclude by induction on the set of threads $\bar{T}$.

- $i \neq i^{\prime}$, any $v$. By R-SendUnBlock the configuration steps to $H ;$; $\iota$.

o. $t \equiv E[K$ obj $\{\overline{f=v} \bar{M}\}]$. We proceed by case analysis on the compatibility of the capability $K$ and the object capability of each value, $\bar{v}$, and $\operatorname{OkRef}(H, K, v)$.

- $\forall v \in \bar{v}$. $\operatorname{OkRef}(H, K, v)$. We proceed by case analysis over $K$

i. $K=$ local. If all values are owned by the current thread, by R-New the configuration steps to $H, \iota \mapsto K$ obj $\{\overline{f=v} \bar{M}\} ; E[\iota]$ where $\iota$ is fresh. If a value is not owned by the current thread, by E-BADINSTANTIATION the configuration reduces to $H ; \operatorname{Err}_{P}$.

ii. $K \neq$ local, by R-New the configuration steps to $H, \iota \mapsto K \operatorname{obj}\{\overline{f=v} \bar{M}\} ; E[\iota]$ where $\iota$ is fresh. 
- $\exists v \in \bar{v} . \neg \operatorname{OkRef}(H, K, v)$ by E-BAdINSTANTIATION the configuration reduces to $H$; $\operatorname{Err}_{P}$.

Theorem 5.2 (Preservation). If $\Gamma \vdash H ; t \bar{T}$ is a well-formed configuration, and $H ; t \bar{T} \leadsto H^{\prime} ; \bar{T}^{\prime} \bar{T}$ then, there exists $a \Gamma^{\prime}$ s.t. $\Gamma^{\prime} \supseteq \Gamma$ and $\Gamma^{\prime} \vdash H^{\prime} ; \overline{T^{\prime}} \bar{T}$

\section{Proof. Initial Assumptions:}

$$
\Gamma \vdash H ; t \bar{T} \quad \text { WF-Configuration }
$$

Step:

$$
H ; t \bar{T} \rightsquigarrow H^{\prime} ; \overline{T^{\prime}} \bar{T} \quad \text { C-Eval }
$$

\section{Need to show:}

$$
\Gamma \vdash H ; \overline{T^{\prime}} \bar{T}
$$

By induction on the shape of the term $t$. If it holds for one thread, then it will hold for all thread configurations. We use the rule C-Eval to reduce a single thread $t$ from the bunch of threads $t \bar{T}$.

1. $t=$ let $x=v$ in $t^{\prime}$. By the induction hypothesis, with rule R-LET

$$
\begin{aligned}
& H ; \text { let } x=v \text { in } t^{\prime} \bar{T} \rightsquigarrow H^{\prime} ; t^{\prime} \bar{T} \\
& \quad \Rightarrow \exists \Gamma^{\prime} \cdot \Gamma^{\prime} \supseteq \Gamma, \Gamma^{\prime} ; H, x \mapsto v \vdash t^{\prime} \bar{T}
\end{aligned}
$$

From initial assumption of well-formedness and WFConfiguration:

$$
\begin{aligned}
& \operatorname{dom}(\Gamma)=\operatorname{dom}(H) \\
& \Gamma \vdash H \\
& \forall t^{\prime \prime} \in \text { let } x=v \text { in } t^{\prime} \bar{T} . \Gamma \vdash H ; t^{\prime \prime} \\
& \text { Local }\left(H, \text { let } x=v \text { in } t^{\prime} \bar{T}\right) \\
& \text { Isolated }\left(H, \text { let } x=v \text { in } t^{\prime} \bar{T}\right)
\end{aligned}
$$

\section{Need to show}

$\exists \Gamma^{\prime} . \Gamma^{\prime} \supseteq \Gamma \wedge \Gamma^{\prime} \vdash H^{\prime} ; t^{\prime} \bar{T}$, where $H^{\prime}=H, x \mapsto v$

The strategy of the proof is to show that all components are well-formed. By rule WF-CONFIGURATION, we need to show:

$$
\begin{aligned}
& \Gamma^{\prime} \supseteq \Gamma \\
& \operatorname{dom}\left(\Gamma^{\prime}\right)=\operatorname{dom}\left(H^{\prime}\right) \\
& \Gamma^{\prime} \vdash H, x \mapsto v \\
& \forall t^{\prime \prime} \in t^{\prime} \bar{T} \cdot \Gamma^{\prime} ; H, x \mapsto v \vdash t^{\prime \prime} \\
& \text { Local }\left(H^{\prime}, t^{\prime} \bar{T}\right) \\
& \text { Isolated }\left(H^{\prime}, t^{\prime} \bar{T}\right)
\end{aligned}
$$

We pick $\Gamma^{\prime}=\Gamma, x: C a p(H, x)$, and $H^{\prime}=H, x \mapsto v$. We now proof each of the components:

- We picked $\Gamma^{\prime}=\Gamma, x: C a p(H, x)$, this trivially satisfies (15), $\Gamma^{\prime} \supseteq \Gamma$.
- Show that $\operatorname{dom}\left(\Gamma^{\prime}\right)=\operatorname{dom}\left(H^{\prime}\right)$. We know that $\Gamma^{\prime}=$ $\Gamma, x: \operatorname{Cap}(H, x)$ and $H^{\prime}=H, x \mapsto v$. To show $\operatorname{dom}(\Gamma, x: \operatorname{Cap}(H, x))=\operatorname{dom}(H, x \mapsto v)$ :

a. $\operatorname{dom}(\Gamma)=\operatorname{dom}(H)$ by assumption (10).

b. Trivially, $x \in \operatorname{dom}(\Gamma, x: \operatorname{Cap}(H, x))$ and $x \in$ $\operatorname{dom}(H, x \mapsto v)$.

From (1a) and (1b), we conclude that $\operatorname{dom}\left(\Gamma^{\prime}\right)=$ $\operatorname{dom}\left(H^{\prime}\right)$.

- Show $\Gamma, x: \operatorname{Cap}(H, x) \vdash H, x \mapsto v(17)$. We proceed by case analysis on the shape of $v$.

- $v=\iota$ (and by our pick of $\Gamma^{\prime}$ we have $x: \operatorname{Cap}(H, x)$ ). We proceed by checking that the environment $\Gamma^{\prime}$ is well-formed w.r.t. $H^{\prime}$.

a. From the assumption of well-formed threads (Eq. (12)) and typing rule WF-LeT: $x \notin \operatorname{dom}(\Gamma)$

b. We prove that $\iota \in \operatorname{dom}\left(H^{\prime}\right)$

i. From thread well-formedness assumption Eq. (12), $\Gamma \vdash H ; t^{\prime \prime}$ for all threads $t^{\prime \prime} \in t \bar{T}$. From this fact and by rule WF-Term, $\Gamma \vdash$ let $x=\iota$ in $t^{\prime}$.

ii. By Lemma B.17 with Item 1(b)i, $\iota \in \operatorname{dom}(\Gamma)$.

iii. From the initial assumption, $\operatorname{dom}(\Gamma)=\operatorname{dom}(H)$.

iv. From Item 1(b)ii and Eq. (10) we conclude that $\iota \in \operatorname{dom}(H)$.

v. From $\Gamma^{\prime}=\Gamma, x:{ }_{-} \supseteq \Gamma$ and the proved step $\operatorname{dom}\left(\Gamma^{\prime}\right)=\operatorname{dom}\left(H^{\prime}\right)$, we can conclude that $\iota \in \operatorname{dom}\left(H^{\prime}\right)$ as $H^{\prime} \supseteq H$ and $\iota \in H$ by 1 (b)iv.

c. From the assumption $\Gamma \vdash H$, by rule WF-HОвјест any $\iota \in \operatorname{dom}(H)$ with capability $K$ must have had $\Gamma(\iota)=K$. By our pick of $\Gamma^{\prime}$ where $x: \operatorname{Cap}(H, \iota)$ and by definition of $\operatorname{Cap}(H, \iota)$, it follows that $\Gamma(x)=\Gamma(\iota)=K$.

d. From assumption (11), $\Gamma \vdash H$.

From steps (1a) - (1d) and by rule WF-H-VAR, we conclude that $\Gamma, x: \operatorname{Cap}(H, x) \vdash H, x \mapsto v$.

$-v=\mathrm{T}$. We need to show that $x \in \operatorname{dom}(\Gamma)$ and $\Gamma^{\prime}+H$.

a. By our pick of $\Gamma^{\prime}$, trivially $x \in \operatorname{dom}\left(\Gamma^{\prime}\right)$,

b. By our assumption that $\Gamma \vdash H(11)$ and $\Gamma^{\prime} \supseteq \Gamma$ and direct application of Lemma B.18, $\Gamma^{\prime} \vdash H$

c. From $1 \mathrm{a}$ and $1 \mathrm{~b}$ and by application of rule WFH-ABSENT, we conclude that $\Gamma^{\prime} \vdash H^{\prime}$.

- Show $\forall t^{\prime \prime} \in t^{\prime} \bar{T} \cdot \Gamma^{\prime} ; H, x \mapsto v \vdash t^{\prime \prime}$. There are multiple cases to consider. For all $t^{\prime \prime} \in \bar{T}$ and by Lemma B.21, extending the environment and heap do not affect the well-formedness of a thread. For the case where $t^{\prime \prime}=t^{\prime}$, we proceed by case analysis on the shape of $t^{\prime}$.

$-t^{\prime}=x$.

a. We just proved $\Gamma, x: \operatorname{Cap}(H, x) \vdash H, x \mapsto v(17)$.

b. Since $x \in \operatorname{dom}(\Gamma, x: \operatorname{Cap}(H, x))$ and by our assumptions of well-formedness $\vdash \Gamma$ and $x \notin \Gamma$, 
by direct application of WF-Env-VAR, $\vdash \Gamma, x$ : $\operatorname{Cap}(H, x)$.

c. From $1 \mathrm{~b}$ and direct application of WF-VAR, $\Gamma, x$ : $C a p(H, x) \vdash x$.

d. From 1a and $1 \mathrm{c}$ and by application of WF-Term, we conclude $\Gamma^{\prime} ; H, x \mapsto v \vdash x$ which is what we had to show.

- $t^{\prime}=$ let $x=e$ in $t^{\prime \prime \prime}$. Follows the same steps as the prove for $t^{\prime}=x$.

- $t^{\prime}=$ consume $x$. Follows the same steps as the prove for $t^{\prime}=x$.

$-t^{\prime}=(K) w$. Follows the same steps as the prove for $t^{\prime}=x$.

- Show Local $\left(H^{\prime}, t^{\prime} \bar{T}\right)$ (19). There are multiple cases to consider, depending on whether there is a local variable and if this one belongs to $t^{\prime}$ or $\bar{T}$

a. No local variables. Then the induction hypothesis is vacuously satisfied, since there is no location to which we can apply it.

b. $\iota \in \operatorname{ROG}\left(H^{\prime}, t^{\prime}\right) \wedge$ isLocal $(H, \iota)$.

i. From the initial assumptions (13) and the Local $(H, t \bar{T})$ and $x \notin \operatorname{dom}(H)$ and $\iota$ is local to $t$ and $H^{\prime}=$ $H, x \mapsto \iota$ and by direct application of Lemma B.22, extending the heap with a fresh variable does not affect other threads. Thus, Local $\left(H^{\prime}, \bar{T}\right)$. With this, we know that $x$ and $\iota$ is still not reachable from other threads.

ii. Now, we need to show that $t^{\prime}$ maintains the thread-locality of $t$ and extending the heap does not allow to reach to local variables from other threads.

We need to show that Local $\left(H^{\prime}, t^{\prime} \bar{T}\right)$. Clearly $t^{\prime} \subseteq t, t^{\prime}$ only has access to the free variables available in $t$, by the assumptions of the case, 1(b)i and by direct application of Lemma B.24, we conclude Local $\left(H^{\prime}, t^{\prime} \bar{T}\right)$.

c. $\iota \notin \operatorname{ROG}\left(H^{\prime}, t^{\prime}\right) \wedge$ isLocal $(H, \iota)$. It must be the case that $\iota$ is local to some other thread. By application of Lemma B.22, we can conclude that Local $\left(H^{\prime}, t^{\prime} \bar{T}\right)$. Thus, we can conclude that Local $\left(H^{\prime}, t^{\prime} \bar{T}\right)$.

- Show Isolated $\left(H^{\prime}, t^{\prime} \bar{T}\right),(20)$. From the initial assumption Isolated $(H$, let $x=v$ in $t \bar{T})(14)$, an isolate location can either be in a field or multiple times in the stack accesible to a single thread. If there are multiple references to it, then it must be on the stack. We consider the case where there are multiple references, as the predicate is vacuously isolated otherwise.

a. Assume islso $(H, v)$. Since there are multiple references we need to further assume its owner. Assume $v$ is only accesible from Isolated $(H$, let $x=v$ in $t)$. Then, extending the environment with a stack variable cannot break isolation, Lemma B.23.
2. $t=E[x]$. By the induction hypothesis,

$H ; E[x] \bar{T} \leadsto H ; \overline{T^{\prime}} \bar{T} \Rightarrow \exists \Gamma^{\prime} . \Gamma^{\prime} \supseteq \Gamma \wedge \Gamma^{\prime} \vdash H^{\prime} ; \overline{T^{\prime}} \bar{T}$

We proceed by case analysis over the step.

R-Var , $H ; E[x] \rightsquigarrow H ; E[v]$.

From initial assumption and WF-CONFIGURATION:

$$
\begin{aligned}
& \Gamma \vdash H ; E[x] \\
& \Gamma \vdash H \\
& \forall t^{\prime} \in E[x] \bar{T} . \Gamma \vdash H ; t^{\prime} \\
& \operatorname{Local}(H, E[x] \bar{T}) \\
& \text { Isolated }(H, E[x] \bar{T})
\end{aligned}
$$

\section{Need to show}

$\exists \Gamma^{\prime} . \Gamma^{\prime} \supseteq \Gamma \wedge \Gamma^{\prime} \vdash H^{\prime} ; E[v] \bar{T}$, where $H^{\prime}=H$

The strategy of the proof is to show that all components are well-formed. By rule WF-Configuration and $\mathrm{IH}$, we need to show:

$$
\begin{aligned}
& \Gamma^{\prime} \supseteq \Gamma \\
& \Gamma^{\prime} \vdash H^{\prime} ; E[v] \\
& \Gamma^{\prime} \vdash H \\
& \forall t^{\prime \prime} \in E[v] \bar{T} \cdot \Gamma^{\prime} \vdash H ; t^{\prime \prime} \\
& \operatorname{Local}\left(H^{\prime}, E[v] \bar{T}\right) \\
& \text { Isolated }\left(H^{\prime}, E[v] \bar{T}\right)
\end{aligned}
$$

We pick $\Gamma^{\prime}=\Gamma$ and $H^{\prime}=H$ by R-VAR. We now proof each of the components of the configuration:

- We picked $\Gamma^{\prime}=\Gamma, x: K$, this trivially satisfies (26).

- Show that $\Gamma^{\prime} \vdash H^{\prime} ; E[v]$ (27).

a. By (21), with WF-LET and WF-Loc, we conclude that $\Gamma^{\prime} \vdash E[v]$ is well-formed

b. By WF-Term-LeT with (2a), we conclude that $\Gamma^{\prime} \vdash H^{\prime} ; E[v]$.

- Show $\Gamma^{\prime} \vdash H$ (28). Trivially satisfied from (22).

- Show $\forall t^{\prime \prime} \in E[v] \bar{T}$. $\Gamma^{\prime} \vdash H ; t^{\prime \prime}$. The case for $\bar{T}$ is trivially from (23). The case for $E[v]$ was shown in above (27).

- Show Local $\left(H^{\prime}, t^{\prime} \bar{T}\right)$ (30). This is trivially satisfied from (24).

- Show Isolated $\left(H^{\prime}, t^{\prime} \bar{T}\right),(31)$. This is trivially satisfied from (25).

By WF-Configuration, we conclude that $\Gamma \vdash H ; E[v] \bar{T}$

E-AbsentVar $H ; E[x] \leadsto H ; \operatorname{Err}_{A}$. From initial assumption and WF-CONFIGURATION:

$$
\begin{aligned}
& \Gamma \vdash H ; E[x] \\
& \Gamma \vdash H
\end{aligned}
$$

By the equivalence rules, we immediately reduce $\operatorname{Err}_{A} \bar{T} \equiv \operatorname{Err}_{A}$.

\section{Need to show}

$\exists \Gamma^{\prime} \cdot \Gamma^{\prime} \supseteq \Gamma \wedge \Gamma^{\prime} \vdash H^{\prime} ; \operatorname{Err}_{A}$, where $H^{\prime}=H$ 
The strategy of the proof is to show that all components are well-formed. By rule WF-Configuration and $\mathrm{IH}$, we need to show:

$$
\begin{aligned}
& \Gamma^{\prime} \supseteq \Gamma \\
& \Gamma^{\prime} \vdash H^{\prime}
\end{aligned}
$$

We pick $\Gamma^{\prime}=\Gamma$ and $H^{\prime}=H$ by R-AbsentVar. We now proof each of the components of the configuration:

- We picked $\Gamma^{\prime}=\Gamma$, this is trivially satisfied by (34).

- Show that $\Gamma^{\prime} \vdash H^{\prime} ; \operatorname{Err}_{A}$. Given that $\Gamma^{\prime}=\Gamma$ and $H=H$, this is trivially satisfied from (33).

We conclude by direct application of WF-CONFIGURATION that $\Gamma \vdash H ; \mathrm{Err}_{A}$ is well-formed.

E-AliasIso $H ; E[x] \rightsquigarrow H$; $\operatorname{Err}_{P}$. From initial assumption and WF-CONFIGURATION:

$$
\Gamma \vdash H
$$

By the equivalence rules, we immediately reduce $\operatorname{Err}_{P} \bar{T} \equiv \operatorname{Err}_{P}$.

Need to show

$$
\exists \Gamma^{\prime} . \Gamma^{\prime} \supseteq \Gamma \wedge \Gamma^{\prime} \vdash H^{\prime} ; \operatorname{Err}_{P} \text {, where } H^{\prime}=H
$$

The strategy of the proof is to show that all components are well-formed. By rule WF-Configuration and $\mathrm{IH}$, we need to show:

$$
\begin{aligned}
& \Gamma^{\prime} \supseteq \Gamma \\
& \Gamma^{\prime} \vdash H^{\prime}
\end{aligned}
$$

We pick $\Gamma^{\prime}=\Gamma$ and $H^{\prime}=H$ by R-AliasIso. We now proof each of the components of the configuration:

- We picked $\Gamma^{\prime}=\Gamma$, this is trivially satisfied from (38).

- Show that $\Gamma^{\prime} \vdash H^{\prime} ; \operatorname{Err}_{A}$. Given that $\Gamma^{\prime}=\Gamma$ and $H=H$, this is trivially satisfied from (37).

We conclude by direct application of WF-CONFIGURATION that $\Gamma \vdash H ; \operatorname{Err}_{P}$ is well-formed.

3. $t=E[$ consume $x]$. By the induction hypothesis,

$$
H ; E[x] \bar{T} \leadsto H ; E[v] \bar{T} \quad \Rightarrow \exists \Gamma^{\prime} . \Gamma^{\prime} \supseteq \Gamma \wedge \Gamma^{\prime} \vdash H^{\prime} ; E[v] \bar{T}
$$

There are two rules, which depend on whether $x$ is $T$ or not.

R-Consume , $H ; E[$ consume $x] \rightsquigarrow H ; E[v]$. From initial assumption and WF-CONFIGURATION:

$$
\begin{aligned}
& \Gamma \vdash H ; E[\text { consume } x] \\
& \Gamma \vdash H \\
& \forall t^{\prime} \in E[\text { consume } x] \bar{T} . \Gamma \vdash H ; t^{\prime} \\
& \operatorname{Local}(H, E[\text { consume } x] \bar{T}) \\
& \text { Isolated }(H, E[\text { consume } x] \bar{T})
\end{aligned}
$$

\section{Need to show:}

$$
\exists \Gamma^{\prime} . \Gamma^{\prime} \supseteq \Gamma \wedge \Gamma^{\prime} \vdash H^{\prime} ; E[v] \bar{T}
$$

The strategy of the proof is to show that all components are well-formed. By rule WF-Configuration and $\mathrm{IH}$, we need to show:

$$
\begin{aligned}
& \Gamma^{\prime} \supseteq \Gamma \\
& \Gamma^{\prime} \vdash H^{\prime} ; E[v] \\
& \Gamma^{\prime} \vdash H \\
& \forall t^{\prime \prime} \in E[v] \bar{T} \cdot \Gamma^{\prime} \vdash H ; t^{\prime \prime} \\
& \text { Local }\left(H^{\prime}, E[v] \bar{T}\right) \\
& \text { Isolated }\left(H^{\prime}, E[v] \bar{T}\right)
\end{aligned}
$$

We pick $\Gamma^{\prime}=\Gamma$ and $H^{\prime}=H$ by R-Consume. We now proof each of the components of the configuration: - We picked $\Gamma^{\prime}=\Gamma$, this trivially satisfies (45).

- Show that $\Gamma^{\prime} \vdash H^{\prime} ; E[v](46)$.

a. By WF-LET and WF-Loc with (40) we conclude that $\Gamma^{\prime} \vdash E[v]$ is well-formed.

b. We need to show that $v=\iota \Rightarrow v \in \operatorname{dom}(H)$. This was part of the initial assumptions in (40), where $x \mapsto \iota \in \operatorname{dom}(H)$.

c. By WF-Term-Let with (3a) and (3b), we can conclude we conclude that $\Gamma^{\prime} \vdash H^{\prime} ; E[v]$ is wellformed.

- Show $\Gamma^{\prime} \vdash H$ (47). Trivially satisfied by (41).

- Show $\forall t^{\prime \prime} \in E[v] \bar{T} \cdot \Gamma^{\prime} \vdash H ; t^{\prime \prime}$. The case for $\bar{T}$ is trivially satisfied by (42). The case for $E[v]$ was shown in above (46).

- Show Local $\left(H^{\prime}, t^{\prime} \bar{T}\right)(49)$. This is trivially satisfied by (43).

- Show Isolated $\left(H^{\prime}, t^{\prime} \bar{T}\right),(50)$. This is trivially satisfied by (44).

By WF-Configuration, we conclude that $\Gamma \vdash H ; E[v] \bar{T}$

E-Consume $H ; E$ [consume $x] \rightsquigarrow H$; $\operatorname{Err}_{A}$ The proof follows the same steps as E-AliasIso.

4. $t=E[x . f]$. By the induction hypothesis,

$$
\begin{aligned}
& H ; E[x . f] \bar{T} \rightsquigarrow H ; \overline{T^{\prime}} \bar{T} \\
& \quad \Rightarrow \exists \Gamma^{\prime} \cdot \Gamma^{\prime} \supseteq \Gamma \wedge \Gamma^{\prime} \vdash H^{\prime} ; \overline{T^{\prime}} \bar{T}
\end{aligned}
$$

There are multiple reductions rules based on whether $x$ maps to $T$ value (E-ABsentTarget), field $f$ does not exist in the object pointed by $x$ (E-NoSuchFiELD), when the field access is to an isolate E-IsoFIELD and when the field access is not to an isolate (R-FIELD). We proceed by case analysis on the step. 
R-Field , $H ; E[x . f] \rightsquigarrow H ; E[v]$. From initial assumption and WF-CONFIGURATION:

$$
\begin{aligned}
& x \mapsto \iota \in H \\
& H(\iota)={ }_{-} \text {obj }\left\{{ }_{-} f=v \bar{M}\right\} \\
& \neg \text { islso }(H, v) \\
& \Gamma \vdash H ; E[x . f] \\
& \Gamma \vdash H \\
& \forall t^{\prime} \in E[x . f] \bar{T} . \Gamma \vdash H ; t^{\prime} \\
& \operatorname{Local}(H, E[x . f] \bar{T}) \\
& \text { Isolated }(H, E[x . f] \bar{T})
\end{aligned}
$$

\section{Need to show:}

$$
\exists \Gamma^{\prime} \cdot \Gamma^{\prime} \supseteq \Gamma \wedge \Gamma^{\prime} \vdash H^{\prime} ; E[v] \bar{T}
$$

The strategy of the proof is to show that all components are well-formed. By rule WF-Configuration and $\mathrm{IH}$, we need to show:

$$
\begin{aligned}
& \Gamma^{\prime} \supseteq \Gamma \\
& \Gamma^{\prime} \vdash H^{\prime} ; E[v] \\
& \Gamma^{\prime} \vdash H \\
& \forall t^{\prime \prime} \in E[v] \bar{T} \cdot \Gamma^{\prime} \vdash H ; t^{\prime \prime} \\
& \operatorname{Local}\left(H^{\prime}, E[v] \bar{T}\right) \\
& \text { Isolated }\left(H^{\prime}, E[v] \bar{T}\right)
\end{aligned}
$$

We pick $\Gamma^{\prime}=\Gamma$ and $H^{\prime}=H$ by R-Field. We now proof each of the components of the configuration:

- We picked $\Gamma^{\prime}=\Gamma$, this trivially satisfies (59).

- Show that $\Gamma^{\prime} \vdash H^{\prime} ; E[v](60)$.

a. By WF-LeT and WF-Loc with (54), we conclude that $\Gamma^{\prime}+E[v]$ is well-formed.

b. By initial assumptions (51), (52), (53), we have that $x \mapsto \iota \in H$ and $\iota \in \operatorname{dom}(H)$ and $H(x . f)=v$ and $v$ is not isolated.

c. By WF-Term-Let with (4a) and (4b), we can conclude we conclude that $\Gamma^{\prime} \vdash H^{\prime} ; E[v]$ is wellformed.

- Show $\Gamma^{\prime} \vdash H$ (61). Trivially satisfied by (55).

- Show $\forall t^{\prime \prime} \in E[v] \bar{T} . \Gamma^{\prime} \vdash H ; t^{\prime \prime}$. The case for $\bar{T}$ is trivially saitisfied by (56). The case for $E[v]$ was shown in above (60).

- Show $\operatorname{Local}\left(H^{\prime}, E[v] \bar{T}\right)(63)$. This is trivially satisfied by (57).

- Show Isolated $\left(H^{\prime}, E[v] \bar{T}\right),(64)$. This is vacuously satisfied by (58) and (53), since $H(x . f)=v$ and $v$ is not an isolate.

By WF-Configuration, we conclude that $\Gamma \vdash H ; E[v] \bar{T}$ is well-formed.

E-NoSuchField $H ; E[x . f] \rightsquigarrow H ; \operatorname{Err}_{N}$
From initial assumption and WF-CONFIGURATION:

$$
\begin{aligned}
& f \text { does not exist in the object } \\
& \Gamma \vdash H
\end{aligned}
$$

By the equivalence rules, we immediately reduce $\operatorname{Err}_{N} \bar{T} \equiv \operatorname{Err}_{N}$. Need to show

$\exists \Gamma^{\prime} . \Gamma^{\prime} \supseteq \Gamma \wedge \Gamma^{\prime} \vdash H^{\prime} ; \operatorname{Err}_{N}$, where $H^{\prime}=H$

The strategy of the proof is to show that all components are well-formed. By rule WF-CONFIGURATION and $\mathrm{IH}$, we need to show:

$$
\begin{aligned}
& \Gamma^{\prime} \supseteq \Gamma \\
& \Gamma^{\prime} \vdash H^{\prime}
\end{aligned}
$$

We pick $\Gamma^{\prime}=\Gamma$ and $H^{\prime}=H$ by E-NoSuchField. We now proof each of the components of the configuration:

- We picked $\Gamma^{\prime}=\Gamma$, this is trivially satisfied by (67).

- Show that $\Gamma^{\prime} \vdash H^{\prime} ; \operatorname{Err}_{N}$. Given that $\Gamma^{\prime}=\Gamma$ and $H=H$, this is trivially satisfied from initial assumption (66).

We conclude by direct application of WF-CONFIGURATION that $\Gamma \vdash H ; \operatorname{Err}_{N}$ is well-formed.

E-AbsentTargetAccess,$H ; E[x . f] \rightsquigarrow H ; \operatorname{Err}_{A}$. From initial assumption and WF-CONFIGURATION:

$$
\begin{aligned}
& H(x)=\top \\
& \Gamma \vdash H
\end{aligned}
$$

By the equivalence rules, we immediately reduce $\operatorname{Err}_{A} \bar{T} \equiv \operatorname{Err}_{A}$. Need to show

$$
\exists \Gamma^{\prime} . \Gamma^{\prime} \supseteq \Gamma \wedge \Gamma^{\prime} \vdash H^{\prime} ; \operatorname{Err}_{A} \text {, where } H^{\prime}=H
$$

The strategy of the proof is to show that all components are well-formed. By rule WF-CONFIGURATION and $\mathrm{IH}$, we need to show:

$$
\begin{aligned}
& \Gamma^{\prime} \supseteq \Gamma \\
& \Gamma^{\prime} \vdash H^{\prime}
\end{aligned}
$$

We pick $\Gamma^{\prime}=\Gamma$ and $H^{\prime}=H$ by E-AbsentTARget Access. We now proof each of the components of the configuration:

- We picked $\Gamma^{\prime}=\Gamma$, this trivially satisfies (72).

- Show that $\Gamma^{\prime} \vdash H^{\prime} ; \operatorname{Err}_{A}$. Given that $\Gamma^{\prime}=\Gamma$ and $H=H$, this is trivially satisfied with (71).

We conclude by direct application of WF-CONFIGURATION that $\Gamma \vdash H ; \operatorname{Err}_{A}$ is well-formed.

E-LocalField,$H ; E[x . f]^{i} \rightsquigarrow H ; \operatorname{Err}_{P}$. From initial assumption and WF-CONFIGURATION:

$$
\begin{aligned}
& H(x . f)=\iota \\
& \text { notLocalOwner }(H, i, \iota) \\
& \Gamma \vdash H
\end{aligned}
$$


By the equivalence rules, we immediately reduce $\operatorname{Err}_{P} \bar{T} \equiv \operatorname{Err}_{P}$. Need to show

$$
\exists \Gamma^{\prime} \cdot \Gamma^{\prime} \supseteq \Gamma \wedge \Gamma^{\prime} \vdash H^{\prime} ; \text { Err }_{P} \text {, where } H^{\prime}=H
$$

The strategy of the proof is to show that all components are well-formed. By rule WF-CONFIGURATION and $\mathrm{IH}$, we need to show:

$$
\begin{aligned}
& \Gamma^{\prime} \supseteq \Gamma \\
& \Gamma^{\prime} \vdash H^{\prime}
\end{aligned}
$$

We pick $\Gamma^{\prime}=\Gamma$ and $H^{\prime}=H$ by E-LocalField. We now proof each of the components of the configuration:

- We picked $\Gamma^{\prime}=\Gamma$, this is trivially satisfied by (77).

- Show that $\Gamma^{\prime} \vdash H^{\prime} ; \operatorname{Err}_{P}$. Given that $\Gamma^{\prime}=\Gamma$ and $H=H$, this is trivially satisfied by (76).

We conclude by direct application of WF-CONFIGURATION that $\Gamma \vdash H ; \operatorname{Err}_{P}$ is well-formed.

E-IsoField , $H ; E[x . f] \leadsto H ; \operatorname{Err}_{P}$. From initial assumption and WF-CONFIGURATION:

$$
\begin{aligned}
& H(x . f)=\iota \\
& \text { islso }(H, \iota) \\
& \Gamma \vdash H
\end{aligned}
$$

By the equivalence rules, we immediately reduce $\operatorname{Err}_{P} \bar{T} \equiv \operatorname{Err}_{P}$. Need to show

$$
\exists \Gamma^{\prime} . \Gamma^{\prime} \supseteq \Gamma \wedge \Gamma^{\prime} \vdash H^{\prime} ; \operatorname{Err}_{P} \text {, where } H^{\prime}=H
$$

The strategy of the proof is to show that all components are well-formed. By rule WF-Configuration and $\mathrm{IH}$, we need to show:

$$
\begin{aligned}
& \Gamma^{\prime} \supseteq \Gamma \\
& \Gamma^{\prime} \vdash H^{\prime}
\end{aligned}
$$

We pick $\Gamma^{\prime}=\Gamma$ and $H^{\prime}=H$ by E-IsoFieLd. We now proof each of the components of the configuration:

- We picked $\Gamma^{\prime}=\Gamma$, this is trivially satisfied by (82).

- Show that $\Gamma^{\prime} \vdash H^{\prime} ; \operatorname{Err}_{p}$. Given that $\Gamma^{\prime}=\Gamma$ and $H=H$, this is trivially satisfied by (81).

We conclude by direct application of WF-CONFIGURATION that $\Gamma \vdash H$; $\operatorname{Err}_{P}$ is well-formed.

5. $t=E[x \cdot f=v]$. By the induction hypothesis,

$$
\begin{aligned}
& H ; E[x . f=v] \bar{T} \rightsquigarrow H ; \overline{T^{\prime}} \bar{T} \\
& \quad \Rightarrow \exists \Gamma^{\prime} \cdot \Gamma^{\prime} \supseteq \Gamma \wedge \Gamma^{\prime} \vdash H^{\prime} ; \overline{T^{\prime}} \bar{T}
\end{aligned}
$$

There are multiple cases to handle, depending on whether the variable $x$ is $T$ (E-ABsentFieldAssign), whether field $f$ exists (E-NoSUChFIELDAssign), whether $x$ is immutable or the capability of $x$ is compatible with the capability of the value $v$ (E-BADFieLDAssign), and finally the case where $x$ is not immutable and the capability of $v$ is compatible with the capability of $x$ (R-FIELDAssign). We proceed by case analysis over the step.
E-AbsentFieldAssign , $H ; E[x . f=v] \leadsto H ; \operatorname{Err}_{A}$. From initial assumption and WF-CONFIGURATION:

$$
\begin{aligned}
& H(x)=\top \\
& \Gamma \vdash H
\end{aligned}
$$

By the equivalence rules, we immediately reduce $\operatorname{Err}_{A} \bar{T} \equiv \operatorname{Err}_{A}$. Need to show

$$
\exists \Gamma^{\prime} . \Gamma^{\prime} \supseteq \Gamma \wedge \Gamma^{\prime} \vdash H^{\prime} ; \operatorname{Err}_{A} \text {, where } H^{\prime}=H
$$

The strategy of the proof is to show that all components are well-formed. By rule WF-Configuration and $\mathrm{IH}$, we need to show:

$$
\begin{aligned}
& \Gamma^{\prime} \supseteq \Gamma \\
& \Gamma^{\prime} \vdash H^{\prime}
\end{aligned}
$$

We pick $\Gamma^{\prime}=\Gamma$ and $H^{\prime}=H$ by E-AbsentFieldAssign. We now proof each of the components of the configuration:

- We picked $\Gamma^{\prime}=\Gamma$, which trivially satisfies (86).

- Show that $\Gamma^{\prime} \vdash H^{\prime} ; \operatorname{Err}_{A}$. Given that $\Gamma^{\prime}=\Gamma$ and $H=H$, this is trivially satisfied with (85).

We conclude by direct application of WF-CONFIGURATION that $\Gamma \vdash H ; \operatorname{Err}_{A}$ is well-formed.

E-BadFieldAssign , $H ; E[x . f=v] \leadsto H ; \operatorname{Err}_{P}$.

Assumptions: From initial assumption and WFCONFIGURATION:

$$
\begin{aligned}
& H(x)=\iota \\
& H(\iota)=K \text { obj }\left\{\_f=v \bar{M}\right\} \\
& \text { isImm }(H, \iota) \vee \neg \operatorname{OkRef}(H, K, v) \\
& \Gamma \vdash H
\end{aligned}
$$

By the equivalence rules, we immediately reduce $\operatorname{Err}_{P} \bar{T} \equiv \operatorname{Err}_{P}$. Need to show

$$
\exists \Gamma^{\prime} . \Gamma^{\prime} \supseteq \Gamma \wedge \Gamma^{\prime} \vdash H^{\prime} ; \operatorname{Err}_{P} \text {, where } H^{\prime}=H
$$

The strategy of the proof is to show that all components are well-formed. By rule WF-CONFIGURATION and $\mathrm{IH}$, we need to show:

$$
\begin{aligned}
& \Gamma^{\prime} \supseteq \Gamma \\
& \Gamma^{\prime} \vdash H^{\prime}
\end{aligned}
$$

We pick $\Gamma^{\prime}=\Gamma$ and $H^{\prime}=H$ by E-BAdFieldAssign. We now proof each of the components of the configuration:

- We picked $\Gamma^{\prime}=\Gamma$, this trivially satisfies (92).

- Show that $\Gamma^{\prime} \vdash H^{\prime} ; \operatorname{Err}_{p}$. Given that $\Gamma^{\prime}=\Gamma$ and $H=H$, this is trivially satisfied by the assumption (91).

We conclude by direct application of WF-CONFIGURATION that $\Gamma \vdash H$; $\operatorname{Err}_{P}$ is well-formed. 
E-NoSuchFieldAssign , $H ; E[x . f=v] \leadsto H ; \operatorname{Err}_{N}$. From initial assumption and WF-CONFIGURATION:

$$
\begin{aligned}
& f \text { does not exist in the object } \\
& \Gamma \vdash H
\end{aligned}
$$

By the equivalence rules, we immediately reduce $\operatorname{Err}_{N} \bar{T} \equiv \operatorname{Err}_{N}$. Need to show

$$
\exists \Gamma^{\prime} . \Gamma^{\prime} \supseteq \Gamma \wedge \Gamma^{\prime} \vdash H^{\prime} ; \text { Err }_{N} \text {, where } H^{\prime}=H
$$

The strategy of the proof is to show that all components are well-formed. By rule WF-CONFIGURATION and $\mathrm{IH}$, we need to show:

$$
\begin{aligned}
& \Gamma^{\prime} \supseteq \Gamma \\
& \Gamma^{\prime} \vdash H^{\prime}
\end{aligned}
$$

We pick $\Gamma^{\prime}=\Gamma$ and $H^{\prime}=H$ by E-NoSuchFieldAssign. We now proof each of the components of the configuration:

- We picked $\Gamma^{\prime}=\Gamma$, this trivially satisfies (96).

- Show that $\Gamma^{\prime} \vdash H^{\prime} ;$ Err $_{N}$. Given that $\Gamma^{\prime}=\Gamma$ and $H=H$, this is trivially satisfied by the assumption (95).

We conclude by direct application of WF-CONFIGURATION that $\Gamma \vdash H ; \operatorname{Err}_{N}$ is well-formed.

R-FieldAssign , $H ; E[x \cdot f=v] \leadsto H ; E\left[v^{\prime}\right]$.

From initial assumption and WF-CONFIGURATION:

$$
\begin{aligned}
& x \mapsto \iota \in H \\
& H(\iota)=K \text { obj }\left\{-f=v^{\prime} \bar{M}\right\} \\
& \neg \text { isImm }(H, \iota) \\
& \text { OkRef }(H, K, v) \\
& \Gamma \vdash H ; E[x . f=v] \\
& \Gamma \vdash H \\
& \forall t^{\prime} \in E[x . f=v] \bar{T} . \Gamma \vdash H ; t^{\prime} \\
& \operatorname{Local}(H, E[x . f=v] \bar{T}) \\
& \text { Isolated }(H, E[x \cdot f=v] \bar{T})
\end{aligned}
$$

\section{Need to show:}

$$
\exists \Gamma^{\prime} . \Gamma^{\prime} \supseteq \Gamma \wedge \Gamma^{\prime} \vdash H^{\prime} ; E\left[v^{\prime}\right] \bar{T}
$$

The strategy of the proof is to show that all components are well-formed. By rule WF-CONFIGURATION and $\mathrm{IH}$, we need to show:

$$
\begin{aligned}
& \Gamma^{\prime} \supseteq \Gamma \\
& \Gamma^{\prime} \vdash H \\
& \Gamma^{\prime} \vdash H^{\prime} ; E\left[v^{\prime}\right] \\
& \forall t^{\prime \prime} \in E\left[v^{\prime}\right] \bar{T} \cdot \Gamma^{\prime} \vdash H ; t^{\prime \prime} \\
& \operatorname{Local}\left(H^{\prime}, E\left[v^{\prime}\right] \bar{T}\right) \\
& \text { Isolated }\left(H^{\prime}, E\left[v^{\prime}\right] \bar{T}\right)
\end{aligned}
$$

We pick $\Gamma^{\prime}=\Gamma$ and $H^{\prime}=H\left[\iota \mapsto K\right.$ obj $\left.\left\{\_f=v^{\prime} \bar{M}\right\}\right]$ by R-FieldAssign. We now proof each of the components of the configuration:

- We picked $\Gamma^{\prime}=\Gamma$, this trivially satisfies (107).

- Show $\Gamma^{\prime} \vdash H^{\prime}$ (108). To do this, it amounts to showing that the object is still well-formed after field substitution.

a. Show that substituting $K$ obj $\left\{_{-} f=v^{\prime} \bar{M}\right\}$ by $K$ obj $\{-f=v \bar{M}\}$ maintains well-formedness. To do this, we first show that all fields in the object satisfy OkRefEnv $\left(\Gamma^{\prime}, K, v\right)$. For all fields where $v \neq v^{\prime}$, this is satisfied by the assumption (102). When $f=v$, with (103) guarantees that $\Gamma(l)=K$, the assumptions of the reduction rule (101) guarantees that $\operatorname{OkRef}(H, K, v)$, and by WF-H-OвJEct with OkRefEnv $(\Gamma, K, v)$, we can conclude that $\Gamma \vdash H, \iota \mapsto K \operatorname{obj}\left\{\_f=v \bar{M}\right\}$ is well-formed.

b. By the well-formed configuration rules with (5a), we conclude that $\Gamma^{\prime} \vdash H^{\prime}$.

- Show that $\Gamma^{\prime} \vdash H^{\prime} ; E[v]$ (109).

a. By the assumption WF-LET and WF-Loc with (102), we conclude that $\Gamma^{\prime} \vdash E[v]$ is well-formed.

b. By the initial assumptions (98), (99), (100), then $x \mapsto \iota \in H$ and $\iota \in \operatorname{dom}(H)$ and $H(x . f)=v^{\prime}$ and $K$ is not immutable.

c. By WF-Term-Let with (5a) and (5b), we can conclude we conclude that $\Gamma^{\prime} \vdash H^{\prime} ; E\left[v^{\prime}\right]$ is wellformed.

- Show $\forall t^{\prime \prime} \in E\left[v^{\prime}\right] \bar{T}$. $\Gamma^{\prime} \vdash H ; t^{\prime \prime}$. The case for $\bar{T}$ is trivially saitisfied by the assumptions, (104). The case for $E[v]$ was shown above (109).

- Show Local $\left(H^{\prime}, E\left[v^{\prime}\right] \bar{T}\right)(111)$. This is trivially satisfied by the assumption (105).

- Show Isolated $\left(H^{\prime}, E\left[v^{\prime}\right] \bar{T}\right),(112)$. This is vacuously satisfied by the assumption (106).

By WF-Configuration, we conclude that $\Gamma \vdash H ; E\left[v^{\prime}\right] \bar{T}$ is well-formed.

6. $t=E[(K) \iota]$. By the induction hypothesis,

$$
\begin{aligned}
& H ; E\left[(K) \iota \bar{T} \rightsquigarrow H ; \overline{T^{\prime}} \bar{T}\right. \\
& \quad \Rightarrow \exists \Gamma^{\prime} . \Gamma^{\prime} \supseteq \Gamma \wedge \Gamma^{\prime} \vdash H^{\prime} ; \overline{T^{\prime}} \bar{T}
\end{aligned}
$$

There are multiple cases to handle, depending on whether the location matches the casted capability or not. We proceed by case analysis over the step.

R-CastLoc,$H ; E[(K) \iota] \rightsquigarrow H ; E[\iota]$. 
From initial assumption and WF-CONFIGURATION:

$$
\begin{aligned}
& H(\iota)=K \operatorname{obj}\left\{{ }_{-}\right\} \\
& \Gamma \vdash H ; E[(K) \iota] \\
& \forall t^{\prime} \in E[(K) \iota] \bar{T} . \Gamma \vdash H ; t^{\prime} \\
& \operatorname{Local}(H, E[(K) \iota] \bar{T}) \\
& \text { Isolated }(H, E[(K) \iota] \bar{T})
\end{aligned}
$$

\section{Need to show:}

$$
\exists \Gamma^{\prime} . \Gamma^{\prime} \supseteq \Gamma \wedge \Gamma^{\prime} \vdash H^{\prime} ; \overline{T^{\prime}} \bar{T}
$$

The strategy of the proof is to show that all components are well-formed. By rule WF-Configuration and $\mathrm{IH}$, we need to show:

$$
\begin{aligned}
& \Gamma^{\prime} \supseteq \Gamma \\
& \Gamma^{\prime} \vdash H \\
& \Gamma^{\prime} \vdash H^{\prime} ; E\left[v^{\prime}\right] \\
& \forall t^{\prime \prime} \in E\left[v^{\prime}\right] \bar{T} . \Gamma^{\prime} \vdash H ; t^{\prime \prime} \\
& \operatorname{Local}\left(H^{\prime}, E\left[v^{\prime}\right] \bar{T}\right) \\
& \text { Isolated }\left(H^{\prime}, E\left[v^{\prime}\right] \bar{T}\right)
\end{aligned}
$$

We pick $\Gamma^{\prime}=\Gamma$, and $H^{\prime}=H$ and $\overline{T^{\prime}}=E[l]$ by RCastLoc. We now proof each of the components of the configuration:

- We picked $\Gamma^{\prime}=\Gamma$, this trivially satisfies (118).

- Show $\Gamma^{\prime} \vdash H^{\prime}$ (119). This is trivially satisfied from (114).

- Show that $\Gamma^{\prime} \vdash H^{\prime} ; E[\iota](120)$. This is trivially satisfied from assumption (114).

- Show $\forall t^{\prime \prime} \in E[\iota] \bar{T} \cdot \Gamma^{\prime} \vdash H ; t^{\prime \prime}$. The case for $\bar{T}$ is trivially satisfied by the assumptions, (115). The case for $E[l]$ was shown above (120).

- Show Local $\left(H^{\prime}, E[l] \bar{T}\right)(122)$. This is trivially satisfied from (116).

- Show Isolated $\left(H^{\prime}, E[\iota] \bar{T}\right),(123)$. This is vacuously satisfied from (117).

By WF-Configuration, we conclude that $\Gamma \vdash H ; E[\iota] \bar{T}$ is well-formed.

E-CastError,$H ; E[(K) \iota] \rightsquigarrow H ; \operatorname{Err}_{C}$.

From initial assumption and WF-ConfigurATION:

$$
\begin{aligned}
& H(\iota)=K^{\prime} \text { obj }\left\{{ }_{--}\right\} \quad \text { where } K \neq K^{\prime} \\
& \Gamma \vdash H
\end{aligned}
$$

By the equivalence rules, we immediately reduce $\operatorname{Err}_{C} \bar{T} \equiv \operatorname{Err}_{C}$. Need to show

$$
\exists \Gamma^{\prime} . \Gamma^{\prime} \supseteq \Gamma \wedge \Gamma^{\prime} \vdash H^{\prime} ; \operatorname{Err}_{N} \text {, where } H^{\prime}=H
$$

The strategy of the proof is to show that all components are well-formed. By rule WF-CONFIGURATION and $\mathrm{IH}$, we need to show:

$$
\begin{aligned}
& \Gamma^{\prime} \supseteq \Gamma \\
& \Gamma^{\prime} \vdash H^{\prime}
\end{aligned}
$$

We pick $\Gamma^{\prime}=\Gamma$ and $H^{\prime}=H$ by E-CAstError. We now proof each of the components of the configuration:

- We picked $\Gamma^{\prime}=\Gamma$, this trivially satisfies (126).

- Show that $\Gamma^{\prime} \vdash H^{\prime}$; $\operatorname{Err}_{C}$. Given that $\Gamma^{\prime}=\Gamma$ and $H=H$, this is trivially satisfied by the assumption (125).

We conclude by direct application of WF-CONFIGURATION that $\Gamma \vdash H ; \operatorname{Err}_{C}$ is well-formed.

7. $t=E[K$ obj $\{\overline{f=v} \bar{M}\}]$. By the induction hypothesis,

$$
\begin{array}{r}
H ; E[K \text { obj }\{\overline{f=v} \bar{M}\}] \bar{T} \rightsquigarrow H ; \overline{T^{\prime}} \bar{T} \\
\Rightarrow \exists \Gamma^{\prime} . \Gamma^{\prime} \supseteq \Gamma \wedge \Gamma^{\prime} \vdash H^{\prime} ; \overline{T^{\prime}} \bar{T}
\end{array}
$$

There are multiple cases to handle, depending on whether object fields are compatible (R-New) or not (E-BADINSTANTIATION). We proceed by case analysis over the step.

R-New , $H ; E[K$ obj $\{\overline{f=v} \bar{M}\}] \leadsto H^{\prime} ; E[\iota]$.

From initial assumption and WF-CONFIGURATION:

$$
\begin{aligned}
& \Gamma \vdash H ; E[K \text { obj }\{\overline{f=v} \bar{M}\}] \\
& \forall v \in \bar{v} . \operatorname{OkRef}(H, K, v) \\
& \forall t^{\prime} \in E[K \text { obj }\{\overline{f=v} \bar{M}\}] \bar{T} . \Gamma \vdash H ; t^{\prime} \\
& \operatorname{Local}(H, E[K \operatorname{obj}\{\overline{f=v} \bar{M}\}] \bar{T}) \\
& \text { Isolated }(H, E[K \text { obj }\{\overline{f=v} \bar{M}\}] \bar{T})
\end{aligned}
$$

\section{Need to show:}

$$
\exists \Gamma^{\prime} . \Gamma^{\prime} \supseteq \Gamma \wedge \Gamma^{\prime} \vdash H^{\prime} ; \overline{T^{\prime}} \bar{T}
$$

The strategy of the proof is to show that all components are well-formed. By rule WF-Configuration and $\mathrm{IH}$, we need to show:

$$
\begin{aligned}
& \Gamma^{\prime} \supseteq \Gamma \\
& \Gamma^{\prime} \vdash H \\
& \Gamma^{\prime} \vdash H^{\prime} ; E[\iota] \\
& \forall t^{\prime \prime} \in E[\iota] \bar{T} \cdot \Gamma^{\prime} \vdash H ; t^{\prime \prime} \\
& \operatorname{Local}\left(H^{\prime}, E[\iota] \bar{T}\right) \\
& \text { Isolated }\left(H^{\prime}, E[\iota] \bar{T}\right)
\end{aligned}
$$

We pick $\Gamma^{\prime}=\Gamma, \iota: K$, and $H^{\prime}=H, \iota \mapsto K$ obj $\{\overline{f=v} \bar{M}\}$ and $\overline{T^{\prime}}=E[\iota]$ by R-New. We now proof each of the components of the configuration:

- We picked $\Gamma^{\prime}=\Gamma, \iota: K$, this trivially satisfies (133).

- Show $\Gamma^{\prime} \vdash H^{\prime}$ (134). To show that $\Gamma, \iota: K \vdash$ $H, \iota \mapsto K$ obj $\{\overline{f=v} \bar{M}\}$, we proceed by proving well-formedness from each component in rule WFН-Овјест. 
a. By assumption 129 values are compatible with the object capability.

b. By assumption 128, all values are in $\Gamma$.

c. From our choosing of $\Gamma^{\prime}, \Gamma^{\prime}(\iota)=K$.

d. By WF-H-ObJect and Helper-OKRefEnv with

(7a) and (7b), we conclude that $\Gamma^{\prime} \vdash H^{\prime}$.

- Show that $\Gamma^{\prime} \vdash H^{\prime} ; E[\iota]$ (135). This is trivially satisfied from assumption (128) with (134).

- Show $\forall t^{\prime \prime} \in E[\iota] \bar{T} . \Gamma^{\prime} \vdash H ; t^{\prime \prime}$. The case for $\bar{T}$ is trivially satisfied by the assumptions, (130). The case for $E[l]$ was shown above (135).

- Show Local $\left(H^{\prime}, E[l] \bar{T}\right)(137)$. This is trivially satisfied by the assumptions (131).

- Show Isolated $\left(H^{\prime}, E[\iota] \bar{T}\right),(138)$. This is satisfied by the assumptions, (132).

By WF-Configuration, we conclude that $\Gamma \vdash H ; E[\iota] \bar{T}$ is well-formed.

E-BadInstantiation , $H ; E[K$ obj $\{\overline{f=v} \bar{M}\}] \leadsto H$; $\operatorname{Err}_{P}$. From initial assumption and WF-CONFIGURATION:

$$
\Gamma \vdash H
$$

By the equivalence rules, we immediately reduce $\operatorname{Err}_{P} \bar{T} \equiv \operatorname{Err}_{P}$. Need to show

$$
\exists \Gamma^{\prime} . \Gamma^{\prime} \supseteq \Gamma \wedge \Gamma^{\prime} \vdash H^{\prime} ; \operatorname{Err}_{P} \text {, where } H^{\prime}=H
$$

The strategy of the proof is to show that all components are well-formed. By rule WF-CONFIGURATION and $\mathrm{IH}$, we need to show:

$$
\begin{aligned}
& \Gamma^{\prime} \supseteq \Gamma \\
& \Gamma^{\prime} \vdash H^{\prime}
\end{aligned}
$$

We pick $\Gamma^{\prime}=\Gamma$ and $H^{\prime}=H$ by E-BADInStantiation. We now proof each of the components of the configuration:

- We picked $\Gamma^{\prime}=\Gamma$, this is trivially satisfied by (140).

- Show that $\Gamma^{\prime} \vdash H^{\prime} ; \operatorname{Err}_{P}$. Given that $\Gamma^{\prime}=\Gamma$ and $H=H$, this is trivially satisfied by the assumption (139).

We conclude by direct application of WF-CONFIGURATION that $\Gamma \vdash H ; \operatorname{Err}_{P}$ is well-formed.

8. $t=E[x \cdot m(v)]$. By the induction hypothesis,

$$
\begin{aligned}
& H ; E[x . m(v)] \bar{T} \rightsquigarrow H ; \overline{T^{\prime}} \bar{T} \\
& \Rightarrow \exists \Gamma^{\prime} . \Gamma^{\prime} \supseteq \Gamma \wedge \Gamma^{\prime} \vdash H^{\prime} ; \overline{T^{\prime}} \bar{T}
\end{aligned}
$$

There are multiple cases to handle, depending on whether object exists (R-CALL) or not (E-ABSENTTARGET) and whether $m$ is a valid method name We proceed by case analysis over the step.
R-Call , $H ; E[x . m(v)] \leadsto H ; E\left[t\left[\operatorname{self}=x^{\prime}\right]\left[y=y^{\prime}\right]\right]$. From initial assumption and WF-CONFIGURATION:

$$
\begin{aligned}
& H(x)=\iota \wedge H(\iota)=K \text { obj }\left\{{ }_{-} \bar{M}\right\} \wedge m \in \bar{M} \\
& \Gamma \vdash H ; E[x \cdot m(v)] \\
& \forall t^{\prime} \in E[x \cdot m(v)] \bar{T} . \Gamma \vdash H ; t^{\prime} \\
& \operatorname{Local}(H, E[x \cdot m(v)] \bar{T}) \\
& \text { Isolated }(H, E[x \cdot m(v)] \bar{T})
\end{aligned}
$$

\section{Need to show:}

$$
\exists \Gamma^{\prime} \cdot \Gamma^{\prime} \supseteq \Gamma \wedge \Gamma^{\prime} \vdash H^{\prime} ; \overline{T^{\prime}} \bar{T}
$$

The strategy of the proof is to show that all components are well-formed. By rule WF-CONFIGURATION and $\mathrm{IH}$, we need to show:

$$
\begin{aligned}
& \Gamma^{\prime} \supseteq \Gamma \\
& \Gamma^{\prime} \vdash H \\
& \Gamma^{\prime} \vdash H^{\prime} ; E\left[t\left[\operatorname{self}=x^{\prime}\right]\left[y=y^{\prime}\right]\right] \\
& \forall t^{\prime \prime} \in E\left[t\left[\operatorname{sel} \mathbf{f}=x^{\prime}\right]\left[y=y^{\prime}\right]\right] \bar{T} \cdot \Gamma^{\prime} \vdash H ; t^{\prime \prime} \\
& \operatorname{Local}\left(H^{\prime}, E\left[t\left[\operatorname{self}=x^{\prime}\right]\left[y=y^{\prime}\right]\right] \bar{T}\right) \\
& \text { Isolated }\left(H^{\prime}, E\left[t\left[\operatorname{self}=x^{\prime}\right]\left[y=y^{\prime}\right]\right] \bar{T}\right)
\end{aligned}
$$

We pick $\Gamma^{\prime}=\Gamma, x^{\prime}: K, y^{\prime}: \Gamma(v)$, and $H^{\prime}=H, x^{\prime} \mapsto$ $\iota, y^{\prime} \mapsto v$ and $\overline{T^{\prime}}=E\left[t\left[\operatorname{self}=x^{\prime}\right]\left[y=y^{\prime}\right]\right]$ by RCALL. We now proof each of the components of the configuration:

- We picked $\Gamma^{\prime}=\Gamma, x^{\prime}: K, y^{\prime}: \Gamma(v)$, this trivially satisfies (147).

- Show $\Gamma^{\prime} \vdash H^{\prime}$ (148). To show that $\Gamma, x^{\prime}: K, y^{\prime}$ : $\Gamma(v) \vdash H, x^{\prime} \mapsto \iota y^{\prime} \mapsto v$, it suffices to show that their capabilities match. By the assumptions (143), $x$ and $\iota$ have capability $K$, and from WF-H-VAR, we conclude that $\Gamma^{\prime} \vdash H, x^{\prime} \mapsto \iota$. Repeating these steps for $y$, lets us conclude $\Gamma^{\prime} \vdash H^{\prime}$.

- Show that $\Gamma^{\prime} \vdash H^{\prime} ; E\left[t\left[\operatorname{self}=x^{\prime}\right]\left[y=y^{\prime}\right]\right]$ (149). This is trivially satisfied from assumption (143) with (148).

- Show $\forall t^{\prime \prime} \in E\left[t\left[\operatorname{self}=x^{\prime}\right]\left[y=y^{\prime}\right]\right] \bar{T} . \Gamma^{\prime} \vdash H ; t^{\prime \prime}$. The case for $\bar{T}$ is trivially satisfied by the assumptions, (144). The case for $E\left[t\left[\operatorname{self}=x^{\prime}\right]\left[y=y^{\prime}\right]\right]$ was shown above (149).

- Show Local $\left(H^{\prime}, E\left[t\left[\operatorname{self}=x^{\prime}\right]\left[y=y^{\prime}\right]\right] \bar{T}\right)(151)$. This is trivially satisfied by the assumptions (145).

- Show Isolated $\left(H^{\prime}, E\left[t\left[\operatorname{self}=x^{\prime}\right]\left[y=y^{\prime}\right]\right] \bar{T}\right),(152)$. This is satisfied by the assumptions, (146). Lets proceed by case analysis over the isolated assumptions and show that it maintains isolateness.

- $\neg$ islso $(H, x)$. Thus, by the assumptions (146), this is trivially satisfied. 
- islso $(H, x) \wedge x \mapsto \iota \in H^{\prime}$. Lets proceed by case analysis over the incoming references being greater than 1 , or not.

* $\left|\operatorname{Inc}\left(H, E\left[t\left[\operatorname{self}=x^{\prime}\right]\left[y=y^{\prime}\right]\right] \bar{T}, \iota\right) \cup\right| \operatorname{Inc}(H, \iota) \mid \leq$ 1. Cannot happen, as $x \mapsto \iota \in H$ already guarantees that the incoming references $\mid \operatorname{lnc}(H, E[t[$ self $=$ $\left.\left.\left.x^{\prime}\right]\left[y=y^{\prime}\right]\right] \bar{T}, \iota\right) \mid \geq 1$

$*\left|\operatorname{Inc}\left(H, E\left[t\left[\operatorname{self}=x^{\prime}\right]\left[y=y^{\prime}\right]\right] \bar{T}, \iota\right) \cup\right| \operatorname{Inc}(H, \iota) \mid \geq$ 1 . Proceed by case analysis over the possible incoming references. We know that $\operatorname{lnc}(H, E[t[$ self $=$ $\left.\left.\left.x^{\prime}\right]\left[y=y^{\prime}\right]\right] \bar{T}, \iota\right) \mid \geq 1$ since $x \mapsto \iota \in H$ and $\iota$ is isolated and we assume (146).

- $|\operatorname{Inc}(H, \iota)| \geq 1$. Cannot happen, by assumption (146) that violates $\operatorname{Inc}(H, \iota)=\emptyset$.

- $\left|\operatorname{Inc}\left(H, E\left[t\left[\operatorname{sel} f=x^{\prime}\right]\left[y=y^{\prime}\right]\right] \bar{T}, \iota\right)\right| \geq 1$. We pick the thread $E\left[t\left[\operatorname{self}=x^{\prime}\right][y=\right.$ $\left.y^{\prime}\right]$ ] and show that it trivially satisfies that $\operatorname{Inc}\left(H, E\left[t\left[\operatorname{sel} \mathbf{f}=x^{\prime}\right]\left[y=y^{\prime}\right]\right] \bar{T}, \iota\right)=\operatorname{Inc}(H, E[t[\operatorname{self}=$ $\left.\left.\left.x^{\prime}\right]\left[y=y^{\prime}\right]\right], \iota\right)$, by the assumption (146), and maintains that $\operatorname{Inc}\left(() H^{\prime}, \iota\right)=\emptyset$. Thus, Isolated $\left(H^{\prime}, E\left[t\left[\operatorname{self}=x^{\prime}\right]\left[y=y^{\prime}\right]\right] \bar{T}\right)$.

By WF-Configuration, we conclude that $\Gamma \vdash H ; E[t[$ self $=$ $\left.\left.x^{\prime}\right]\left[y=y^{\prime}\right]\right] \bar{T}$ is well-formed.

E-NoSuchMethod, $H ; E[x . m(v)] \leadsto H ; \operatorname{Err}_{N}$. This proceeds in the same fashion as E-NoSuchField

E-AbsentTarget , $H ; E[x . m(v)] \leadsto H ; \operatorname{Err}_{A}$. This proceeds in the same fashion as E-ABSENTVAR

9. $t=E\left[\right.$ spawn $\left.(x)\left\{t^{\prime}\right\}\right]$. By the induction hypothesis,

$$
\begin{array}{r}
H ; E\left[\text { spawn }(x)\left\{t^{\prime}\right\}\right] \bar{T} \rightsquigarrow H ; \overline{T^{\prime}} \bar{T} \\
\Rightarrow \exists \Gamma^{\prime} \cdot \Gamma^{\prime} \supseteq \Gamma \wedge \Gamma^{\prime} \vdash H^{\prime} ; \overline{T^{\prime}} \bar{T}
\end{array}
$$

There is only a single reduction rule.

R-Spawn , $H ; E\left[\right.$ spawn $\left.(x)\left\{t^{\prime}\right\}\right] \leadsto H ; E[l] t^{\prime}$. From initial assumption and WF-CONFIGURATION:

$$
\begin{aligned}
& \Gamma \vdash H ; E\left[\text { spawn }(x)\left\{t^{\prime}\right\}\right] \bar{T} \\
& \forall t^{\prime} \in E\left[\text { spawn }(x)\left\{t^{\prime}\right\}\right] \bar{T} . \Gamma \vdash H ; t^{\prime} \\
& \operatorname{Local}\left(H, E\left[\text { spawn }(x)\left\{t^{\prime}\right\}\right] \bar{T}\right) \\
& \text { Isolated }\left(H, E\left[\text { spawn }(x)\left\{t^{\prime}\right\}\right] \bar{T}\right)
\end{aligned}
$$

\section{Need to show:}

$$
\exists \Gamma^{\prime} . \Gamma^{\prime} \supseteq \Gamma \wedge \Gamma^{\prime} \vdash H^{\prime} ; \overline{T^{\prime}} \bar{T}
$$

The strategy of the proof is to show that all components are well-formed. By rule WF-CONFIGURATION and $\mathrm{IH}$, we need to show:

$$
\begin{aligned}
& \Gamma^{\prime} \supseteq \Gamma \\
& \Gamma^{\prime} \vdash H^{\prime} \\
& \Gamma^{\prime} \vdash H^{\prime} ; E[\iota] t^{\prime} \\
& \forall t^{\prime \prime} \in E[\iota] t^{\prime} \bar{T} \cdot \Gamma^{\prime} \vdash H ; t^{\prime \prime} \\
& \operatorname{Local}\left(H^{\prime}, E[\iota] t^{\prime} \bar{T}\right) \\
& \text { Isolated }\left(H^{\prime}, E[\iota] t^{\prime} \bar{T}\right)
\end{aligned}
$$

We pick $\Gamma^{\prime}=\Gamma, \iota$ : local, $x:$ local, and $H^{\prime}=$ $H, x^{\prime} \mapsto \iota, \iota \mapsto \operatorname{chan}\{i, \varnothing\}$ and $\overline{T^{\prime}}=E[\iota] t^{\prime}$ by RSpAwn. We now proof each of the components of the configuration:

- We picked $\Gamma^{\prime}=\Gamma, \iota$ : local, $x$ : local, this trivially satisfies (157).

- Show $\Gamma^{\prime} \vdash H^{\prime}$ (158). By the assumption (153), we have $\Gamma \vdash H$. We proceed by proving that each component is well-formed.

a. $\operatorname{Gamma}(x)=\Gamma(\iota)=$ local

b. By WF-H-VAR with (9a), we conclude that $\Gamma^{\prime} \vdash$ $H, x \mapsto \iota$

c. By WF-H-CHAIN with (9a) and (9b), we conclude that $\Gamma^{\prime} \vdash H^{\prime}$.

- Show that $\Gamma^{\prime} \vdash H^{\prime} ; E[l] t^{\prime}$ (159). This is trivially satisfied from assumption (153) with (158), given that it is part of the assumptions that t' is wellformed from WF-TERM-LET.

- Show $\forall t^{\prime \prime} \in E[\iota] t^{\prime} \bar{T}$. $\Gamma^{\prime} \vdash H ; t^{\prime \prime}$. The case for $\bar{T}$ is trivially satisfied by the assumptions, (154). The case for $E[l]$ was shown above (159).

- Show Local $\left(H^{\prime}, E[\iota] t^{\prime} \bar{T}\right)(161)$. This is vacuously satisfied by the assumption (155).

- Show Isolated $\left(H^{\prime}, E[l] t^{\prime} \bar{T}\right),(162)$. This is satisfied by the assumption (156), since spawning a thread does not affect the Isolated predicate.

By WF-Configuration, we conclude that $\Gamma \vdash H ; E[\iota] t^{\prime} \bar{T}$ is well-formed.

10. $t=E[\leftarrow \iota]$. By the induction hypothesis,

$$
\begin{aligned}
H ; E[ & \leftarrow \iota] \bar{T} \leadsto H ; \overline{T^{\prime}} \bar{T} \\
& \Rightarrow \exists \Gamma^{\prime} . \Gamma^{\prime} \supseteq \Gamma \wedge \Gamma^{\prime} \vdash H^{\prime} ; \overline{T^{\prime}} \bar{T}
\end{aligned}
$$

There are multiple rules, depending on whether the location maps to an object E-REcvBADTARGET or to a channel (R-Recv).

R-RecvBadTarget , $H ; E[\leftarrow \iota] \leadsto H$; $\operatorname{Err}_{N}$. This proof is similar to E-ABSENTVAR, except that $H(\iota)$ maps to an object, instead of to $T$. 
R-Recv , $H ; E[\leftarrow \iota] \leadsto H ; E\left[\iota^{\prime}\right]$. From initial assumption and WF-CoNFIgURATION:

$$
\begin{aligned}
& \Gamma \vdash H ; E[\leftarrow \iota] \bar{T} \\
& \forall t^{\prime} \in E[\leftarrow \iota] \bar{T} . \Gamma \vdash H ; t^{\prime} \\
& \operatorname{Local}(H, E[\leftarrow \iota] \bar{T}) \\
& \text { Isolated }(H, E[\leftarrow \iota] \bar{T})
\end{aligned}
$$

\section{Need to show:}

$$
\exists \Gamma^{\prime} . \Gamma^{\prime} \supseteq \Gamma \wedge \Gamma^{\prime} \vdash H^{\prime} ; \overline{T^{\prime}} \bar{T}
$$

The strategy of the proof is to show that all components are well-formed. By rule WF-Configuration and $\mathrm{IH}$, we need to show:

$$
\begin{aligned}
& \Gamma^{\prime} \supseteq \Gamma \\
& \Gamma^{\prime} \vdash H^{\prime} \\
& \Gamma^{\prime} \vdash H^{\prime} ; E\left[\iota^{\prime}\right] \\
& \forall t^{\prime \prime} \in E\left[\iota^{\prime}\right] \bar{T} . \Gamma^{\prime} \vdash H ; t^{\prime \prime} \\
& \operatorname{Local}\left(H^{\prime}, E\left[\iota^{\prime}\right] \bar{T}\right) \\
& \text { Isolated }\left(H^{\prime}, E\left[\iota^{\prime}\right] \bar{T}\right)
\end{aligned}
$$

We pick $\Gamma^{\prime}=\Gamma$, and $H^{\prime}=H[\iota \mapsto \operatorname{chan}\{i, \varnothing\}]$ and $\overline{T^{\prime}}=E\left[\iota^{\prime}\right]$ by R-Recv. We now proof each of the components of the configuration:

- We picked $\Gamma^{\prime}=\Gamma$, this trivially satisfies (167).

- Show $\Gamma^{\prime} \vdash H^{\prime}$ (168). By (163), all the locations and variables match the expected capability and are defined in $\Gamma$ and $H$. By WF-H-Class with initial assumptions, we conclude $\Gamma^{\prime} \vdash H^{\prime}$ is well-formed

- Show that $\Gamma^{\prime} \vdash H^{\prime} ; E\left[\iota^{\prime}\right]$ (169). By WF-Term-LeT with (163) and (168) where $\iota^{\prime} \in \operatorname{dom}(H)$, we conclude $\Gamma^{\prime} \vdash H^{\prime} ; E\left[\iota^{\prime}\right]$.

- Show $\forall t^{\prime \prime} \in E\left[\iota^{\prime}\right] \bar{T}$. $\Gamma^{\prime} \vdash H ; t^{\prime \prime}$. The case for $\bar{T}$ is trivially satisfied from (164). The case for $E[l]$ was shown above (169).

- Show Local $\left(H^{\prime}, E\left[\iota^{\prime}\right] \bar{T}\right)(171)$. This is trivially satisfied from (165). Explanation: Before the reduction step, by definition of $R O G$, locations leading to channels are not considered part of a ROG. Thus, $\iota^{\prime}$ was unreachable by any thread. By initial assumption of well-formedness $\Gamma \vdash H$ and $\Gamma\left(\iota^{\prime}\right) \neq$ local Thus, $\iota^{\prime}$ is in a single thread, and we can conclude that it satisfies Local $\left(H^{\prime}, E\left[\iota^{\prime}\right] \bar{T}\right)$.

- Show Isolated $\left(H^{\prime}, E\left[\iota^{\prime}\right] \bar{T}\right),(172)$. This is satisfied from initial assumption (166). Explanation: From initial assumption and the helper function Isolated, if $\iota^{\prime}$ is an isolate, then it can only be in a channel. By taking a step and removing $\iota^{\prime}$ from the channel, we place it in the stack and the incoming number of references is 1 , so it vacuously satisfies Isolated $\left(H^{\prime}, E\left[\iota^{\prime}\right] \bar{T}\right)$.
By WF-Configuration, we conclude that $\Gamma \vdash H ; E\left[\iota^{\prime}\right] \bar{T}$ is well-formed.

11. $t=E[\iota \leftarrow v]$. By the induction hypothesis,

$$
\begin{aligned}
H ; E[\iota & \leftarrow v] \bar{T} \rightsquigarrow H ; \overline{T^{\prime}} \bar{T} \\
& \Rightarrow \exists \Gamma^{\prime} . \Gamma^{\prime} \supseteq \Gamma \wedge \Gamma^{\prime} \vdash H^{\prime} ; \overline{T^{\prime}} \bar{T}
\end{aligned}
$$

There are multiple rules, depending on whether the element to send contains local elements (E-SENDINGLOCAL), whether $\iota$ is a channel or not (R-SENDBLOCK and ESendBadTargetOrARgument) and whether $v=\iota^{\prime}$ and maps to a channel (E-SENDBADTARgETORARguMENT)). R-SendBlock , $H ; E[\iota \leftarrow v] \leadsto H ; E\left[\mathbf{\square}_{i} \iota\right]$. From initial assumption and WF-CONFIGURATION:

$$
\begin{aligned}
& \Gamma \vdash H ; E[\iota \leftarrow v] \bar{T} \\
& \forall t^{\prime} \in E[\iota \leftarrow v] \bar{T} . \Gamma \vdash H ; t^{\prime} \\
& \operatorname{Local}(H, E[\iota \leftarrow v] \bar{T}) \\
& \text { Isolated }(H, E[\iota \leftarrow v] \bar{T})
\end{aligned}
$$

\section{Need to show:}

$$
\exists \Gamma^{\prime} . \Gamma^{\prime} \supseteq \Gamma \wedge \Gamma^{\prime} \vdash H^{\prime} ; \overline{T^{\prime}} \bar{T}
$$

The strategy of the proof is to show that all components are well-formed. By rule WF-CONFIGURATION and $\mathrm{IH}$, we need to show:

$$
\begin{aligned}
& \Gamma^{\prime} \supseteq \Gamma \\
& \Gamma^{\prime} \vdash H^{\prime} \\
& \Gamma^{\prime} \vdash H^{\prime} ; E[\iota] \\
& \forall t^{\prime \prime} \in E[\iota] \bar{T} \cdot \Gamma^{\prime} \vdash H ; t^{\prime \prime} \\
& \text { Local }\left(H^{\prime}, E[\iota] \bar{T}\right) \\
& \text { Isolated }\left(H^{\prime}, E[\iota] \bar{T}\right)
\end{aligned}
$$

We pick $\Gamma^{\prime}=\Gamma$, and $H^{\prime}=H[\iota \mapsto \operatorname{chan}\{i, v\}]$ and $\overline{T^{\prime}}=E[\iota]$ by R-SENDBLOCK. We now proof each of the components of the configuration:

- We picked $\Gamma^{\prime}=\Gamma$, this trivially satisfies (177).

- Show $\Gamma^{\prime} \vdash H^{\prime}$ (178). From (173), all the locations and variables match the expected capability and are defined in $\Gamma$ and $H$. By WF-H-CHAN with the initial assumptions, we conclude $\Gamma^{\prime} \vdash H^{\prime}$ is wellformed.

- Show that $\Gamma^{\prime} \vdash H^{\prime} ; E[\iota]$ (179). By WF-Term-Let with (173) and (178) where $\iota \in \operatorname{dom}(H)$, we conclude $\Gamma^{\prime} \vdash H^{\prime} ; E[\iota]$.

- Show $\forall t^{\prime \prime} \in E[\iota] \bar{T} \cdot \Gamma^{\prime} \vdash H ; t^{\prime \prime}$. The case for $\bar{T}$ is trivially satisfied from (174). The case for $E[\iota]$ was shown above (179).

- Show Local $\left(H^{\prime}, E[\iota] \bar{T}\right)(181)$. This is trivially satisfied from (175).

- Show Isolated $\left(H^{\prime}, E[\iota] \bar{T}\right),(182)$. This is satisfied from initial assumption (176). Explanation: From initial assumption and the helper function Isolated, 
if $v$ is an isolate, then it can only be in the stack and is unique. By taking a step and removing $v$ from the stack, we place it in channel. The number of stack incoming references for $v$ is 0 , and the number of heap incoming references is 1 . Thus, the configuration vacuously satisfies Isolated $\left(H^{\prime}, E[\iota] \bar{T}\right)$, as the antecedent of the implication is false.

By WF-Configuration, we conclude that $\Gamma \vdash H ; E[\iota] \bar{T}$ is well-formed.

E-SendBadTargetOrArgument , $H ; E\left[\iota \leftarrow \iota^{\prime}\right] \rightsquigarrow$

$H ; \operatorname{Err}_{N}$. This proof follows the same structure of E-RecvBADTARget

E-SendingLocal,$H ; E[v \leftarrow \iota] \rightsquigarrow H$; $\operatorname{Err}_{P}$. This proof follows the same structure of E-BADINSTANTIATION 12. $t=E\left[\mathbf{\Xi}_{i} l\right]$. By the induction hypothesis,

$$
\begin{aligned}
H ; E\left[\mathbf{\Xi}_{i} \iota\right] & \bar{T} \rightsquigarrow H ; \overline{T^{\prime}} \bar{T} \\
& \Rightarrow \exists \Gamma^{\prime} \cdot \Gamma^{\prime} \supseteq \Gamma \wedge \Gamma^{\prime} \vdash H^{\prime} ; \overline{T^{\prime}} \bar{T}
\end{aligned}
$$

There is a single reduction rule that can make progress, R-SENDUNBLOCK.

R-SendUnblock,$H ; E\left[\mathbf{a}_{i} \iota\right] \leadsto H^{\prime} ; E[\iota]$. From initial assumption and WF-CONFIGURATION:

$$
\begin{aligned}
& \Gamma \vdash H ; E\left[\mathbf{a}_{i} \iota\right] \bar{T} \\
& \forall t^{\prime} \in E\left[\mathbf{\sigma}_{i} l\right] \bar{T} . \Gamma \vdash H ; t^{\prime} \\
& \operatorname{Local}\left(H, E\left[\mathbf{\square}_{i} \iota\right] \bar{T}\right) \\
& \text { Isolated }\left(H, E\left[\mathbf{\square}_{i} \iota\right] \bar{T}\right)
\end{aligned}
$$

\section{Need to show:}

$$
\exists \Gamma^{\prime} . \Gamma^{\prime} \supseteq \Gamma \wedge \Gamma^{\prime} \vdash H^{\prime} ; \overline{T^{\prime}} \bar{T}
$$

The strategy of the proof is to show that all components are well-formed. By rule WF-CONFIGURATION, we need to show:

$$
\begin{aligned}
& \Gamma^{\prime} \supseteq \Gamma \\
& \Gamma^{\prime} \vdash H^{\prime} \\
& \Gamma^{\prime} \vdash H^{\prime} ; E[\iota] \\
& \forall t^{\prime \prime} \in E[\iota] \bar{T} \cdot \Gamma^{\prime} \vdash H ; t^{\prime \prime} \\
& \operatorname{Local}\left(H^{\prime}, E[\iota] \bar{T}\right) \\
& \text { Isolated }\left(H^{\prime}, E[\iota] \bar{T}\right)
\end{aligned}
$$

We pick $\Gamma^{\prime}=\Gamma$, and $H^{\prime}=H$ and $\overline{T^{\prime}}=E[\iota]$ by R-SENDUNBLOCK. We now proof each of the components of the configuration:

- We picked $\Gamma^{\prime}=\Gamma$, this trivially satisfies (187).

- Show $\Gamma^{\prime} \vdash H^{\prime}$ (188). This is part of the initial assumptions.

- Show that $\Gamma^{\prime} \vdash H^{\prime} ; E[l]$ (189). This was part of the initial assumptions (183).
- Show $\forall t^{\prime \prime} \in E[l] \bar{T} \cdot \Gamma^{\prime} \vdash H ; t^{\prime \prime}$. The case for $\bar{T}$ is trivially satisfied from (184). The case for $E[\iota]$ was shown above (189).

- Show Local $\left(H^{\prime}, E[\iota] \bar{T}\right)(191)$. This is trivially satisfied from the initial assumption (185).

- Show Isolated $\left(H^{\prime}, E[\iota] \bar{T}\right),(192)$. This is satisfied from initial assumption (186), as $\Gamma(\iota)=$ local.

By WF-Configuration, we conclude that $\Gamma \vdash H ; E[l] \bar{T}$ is well-formed.

13. $t=E[K \operatorname{copy} x]$. By the induction hypothesis,

$$
\begin{aligned}
& H ; E[K \text { copy } x] \bar{T} \rightsquigarrow H ; \overline{T^{\prime}} \bar{T} \\
& \quad \Rightarrow \exists \Gamma^{\prime} . \Gamma^{\prime} \supseteq \Gamma \wedge \Gamma^{\prime} \vdash H^{\prime} ; \overline{T^{\prime}} \bar{T}
\end{aligned}
$$

There are two reduction rules, which depend on the value in $x$.

R-Copy , $H ; E[K \operatorname{copy} x] \rightsquigarrow H^{\prime} ; E\left[\iota^{\prime}\right]$.

From initial assumption and WF-CONFIGURATION:

$$
\begin{aligned}
& \Gamma \vdash H ; E[K \text { copy } x] \bar{T} \\
& \forall t^{\prime} \in E[K \text { copy } x] \bar{T} . \Gamma \vdash H ; t^{\prime} \\
& \operatorname{Local}(H, E[K \text { copy } x] \bar{T}) \\
& \text { Isolated }(H, E[K \text { copy } x] \bar{T})
\end{aligned}
$$

\section{Need to show:}

$$
\exists \Gamma^{\prime} \cdot \Gamma^{\prime} \supseteq \Gamma \wedge \Gamma^{\prime} \vdash H^{\prime} ; \overline{T^{\prime}} \bar{T}
$$

The strategy of the proof is to show that all components are well-formed. By rule WF-CONFIGURATION and $\mathrm{IH}$, we need to show:

$$
\begin{aligned}
& \Gamma^{\prime} \supseteq \Gamma \\
& \Gamma^{\prime} \vdash H^{\prime} \\
& \Gamma^{\prime} \vdash H^{\prime} ; E\left[\iota^{\prime}\right] \\
& \forall t^{\prime \prime} \in E\left[\iota^{\prime}\right] \bar{T} \cdot \Gamma^{\prime} \vdash H ; t^{\prime \prime} \\
& \operatorname{Local}\left(H^{\prime}, E[\iota] \bar{T}\right) \\
& \text { Isolated }\left(H^{\prime}, E[\iota] \bar{T}\right)
\end{aligned}
$$

We pick $\Gamma^{\prime}$ to be:

$$
\begin{aligned}
\Gamma^{\prime}= & \left\{x: K \mid x \in \operatorname{dom}(H) \wedge H(x)=\iota \wedge H(\iota)=K \text { obj }\left\{{ }_{--}\right\}\right\} \\
& \cup\left\{\iota: K \mid \iota \in \operatorname{dom}(H) \wedge H(\iota)=K \text { obj }\left\{_{--}\right\}\right\} \\
& \cup\{\iota: \operatorname{local} \mid \iota \in \operatorname{dom}(H) \wedge H(\iota)=\operatorname{chan}\{i, v\}\}
\end{aligned}
$$

and $H^{\prime}=H, H^{\prime \prime}$ where $H^{\prime \prime}$ is a copy of the $R O G$ with fresh location names and where field values in the $R O G$ refer to the updated fresh locations. $\overline{T^{\prime}}=E\left[\iota^{\prime}\right]$ by R-Copy. We now proof each of the components of the configuration:

- Clearly, $\Gamma^{\prime} \supseteq \Gamma$ and satisfies (197).

- Show $\Gamma^{\prime} \vdash H^{\prime}$ (198). Trivial by direct application of WF-H-OBJEct, WF-H-VAR, and WF-H-CHAN. 
- Show that $\Gamma^{\prime} \vdash H^{\prime} ; E\left[\iota^{\prime}\right]$ (199). By WF-Term-Let with (193) and (198) where ROG $\left(H^{\prime}, \iota^{\prime}\right) \subseteq \operatorname{dom}(H)$, we conclude $\Gamma^{\prime} \vdash H^{\prime} ; E[\iota]$.

- Show $\forall t^{\prime \prime} \in E\left[\iota^{\prime}\right] \bar{T}$. $\Gamma^{\prime} \vdash H^{\prime} ; t^{\prime \prime}$. The case for $\bar{T}$ is trivially satisfied from (194). The case for $E\left[\iota^{\prime}\right]$ was shown above (199).

- Show Local $\left(H^{\prime}, E\left[\iota^{\prime}\right] \bar{T}\right)(201)$. This is trivially satisfied since it returns a new copy of the objects.

- Show Isolated $\left(H^{\prime}, E\left[\iota^{\prime}\right] \bar{T}\right),(202)$. This is trivially satisfied by the initial assumptions, since it only extends the store with a new copy of the objects.

By WF-Configuration, we conclude that $\Gamma \vdash H ; E\left[\iota^{\prime}\right] \bar{T}$ is well-formed.

E-AbsentCopyTarget, $H ; E[K$ copy $x] \leadsto H ; \operatorname{Err}_{A}$. This proof follows the same structure as E-ABSENTVAR.

Theorem 5.5 (Dalarna is Data-Race Free Modulo Unsafe Objects). All data races directly or indirectly involve an unsafe object. A data race is defined as a read/write or write/write access to an object by different threads without interleaving synchronisation (In our formalism, this means an interleaving transfer of an object to another thread; formal proof in Appendix).

Proof. From Theorem 5.2 (Preservation), and WF-ConfigURATION, every configuration satisfies the Local and Isolated predicates. This means that at no point will a local object be reachable from two threads, which excludes these from taking part in data-races. By R-FieldAssign and E-BADFIELdAssign, immutable objects cannot be modified, which excludes these from taking part in data-races. This leaves isolated objects. For a data-race to happen on an isolated object $l$, the following must be possible: at time $t_{0}$, a thread reads/writes $\iota$, at time $t_{1}>t_{0}$ (later reduction step), another thread read$\mathrm{s} /$ writes $\iota$, and nowhere between $t_{0}$ and $t_{1}$ is $\iota$ moved from the first thread to the second. Because of Isolated, if there are more than one reachable ${ }^{13}$ pointers to $l$, all those pointers are on the stack of a single thread. Thus, for two threads to be able to reach the same isolated object without moving it, the isolated object must be stored in a field $f$ in an object $o$ reachable from both threads (WLOG, assume directly reachable from stack as we require unravelling paths fieldby-field). From before, we know $o$ cannot be local or isolated. Immutable objects cannot contain isolated objects (R-NEw and OkRef). Thus, this is only possible when $o$ is unsafe. Thus, we conclude that Dalarna is data-race free modulo unsafe objects.

Theorem 5.6 (Dynamic Gradual Guarantee). Let $H ; t \overline{T_{0}}$ be a configuration and $\Gamma$ a store type such that $\Gamma+H ; t \overline{T_{0}}$. Let ${ }^{e}$ be a function that replaces safe capabilities with unsafe in heaps, terms, etc (Definition B.11). Then:

\footnotetext{
${ }^{13}$ Note that as we do not discard old stack variables, there may be variables in the store which are unreachable to the program.
}

1. $\Gamma^{e} \vdash\left(H ; t \overline{T_{0}}\right)^{e}$.

2. a. If $H ; t \overline{T_{0}} \leadsto H^{\prime} ; \overline{T_{1}} \overline{T_{0}}$ and $\overline{T_{1}} \neq$ Err then, $\left(H ; t \overline{T_{0}}\right)^{e} \leadsto\left(H^{\prime} ; \overline{T_{1}} \overline{T_{0}}\right)^{e}$.

b. If $H ; t \overline{T_{0}} \rightsquigarrow H^{\prime} ; \overline{T_{1}} \overline{T_{0}}$ and $\overline{T_{1}}=\operatorname{Err}_{A} \vee$ Err $_{N}$ then, $(H ; t \bar{T})^{e} \leadsto H^{\prime \prime} ; \bar{T}_{1} \bar{T}_{0}$

3.a. If $\left(H ; t \overline{T_{0}}\right)^{e} \leadsto\left(H^{\prime} ; \operatorname{Err} \overline{T_{0}}\right)^{e}$ then, $H ; t \overline{T_{0}} \leadsto H^{\prime} ; E r r \overline{T_{0}}$

b. If $\left(H ; t \overline{T_{0}}\right)^{e} \rightsquigarrow\left(H^{\prime} ; \bar{T} \overline{T_{0}}\right)^{e}$ and $\bar{T} \neq$ Err then, $H ; t \overline{T_{0}} \rightsquigarrow H^{\prime} ; \overline{T^{\prime}} \overline{T_{0}}$ and $\overline{T^{\prime}}=\operatorname{Err}_{P} \vee \operatorname{Err}_{C} \vee \bar{T}$.

Proof. $\quad$ 1. $\Gamma \vdash H ; t \overline{T_{0}} \Rightarrow \Gamma^{e} \vdash\left(H ; t \overline{T_{0}}\right)^{e}$.

This is trivially satisfied from the definition of capability stripping (Definition B.11). Any configuration with safe capabilities can replace the safe capabilities by unsafe and will still be well-formed.

2. a. If $H ; t \overline{T_{0}} \rightsquigarrow H^{\prime} ; \overline{T_{1}} \overline{T_{0}}$ and $\overline{T_{1}} \neq \operatorname{Err}$ then, $\left(H ; t \overline{T_{0}}\right)^{e} \leadsto$ $\left(H^{\prime} ; \overline{T_{1}} \overline{T_{0}}\right)^{e}$.

By induction on a derivation of $H ; t \overline{T_{0}} \rightsquigarrow H^{\prime} ; \overline{T_{1}} \overline{T_{0}}$. We need to show:

$$
\left(H ; t \overline{T_{0}}\right)^{e} \rightsquigarrow\left(H^{\prime} ; \overline{T_{1}} \overline{T_{0}}\right)^{e}
$$

From the induction hypothesis, we assume that the reduction rule does not produce an error, i.e., $T_{1} \neq$ $E r r$, and that the term to reduce is fixed, i.e., $t$.

i. R-LET. $H$; let $x=v$ in $t \overline{T_{0}} \rightsquigarrow H, x \mapsto v ; t \overline{T_{0}}$

A. $(H ; \text { let } x=v \text { in } t)^{e}=(H)^{e}$; let $x=v$ in $t$ by Lemma B.26 with $t=$ let $x=v$ in $t$.

B. $\left(H ; \text { let } x=v \text { in } t \overline{T_{0}}\right)^{e} \rightsquigarrow\left(H, x \mapsto v ; t \overline{T_{0}}\right)^{e}$ by application C-EvAL, R-LET with Item 2(a)iA.

ii. R-VAR. $H ; E[x] \overline{T_{0}} \leadsto H ; E[v] \overline{T_{0}}$

A. ᄀislso (...) by the assumptions.

B. $(H ; E[x])^{e}=(H)^{e} ; E^{\prime}[x]$ where $E^{\prime}[\bullet]=\left(E^{\prime}\right)^{e}[\bullet]$ by Lemma B.26 with $t=E[x]$.

C. $\left(H ; E[x] \overline{T_{0}}\right)^{e} \rightsquigarrow\left(H ; E[v] \overline{T_{0}}\right)^{e}$ by application C-Eval, R-VAR with Item 2(a)iiB.

iii. R-Consume. $H ; E[$ consume $x] \overline{T_{0}} \rightsquigarrow H, x \mapsto \mathrm{T} ; E[v] \overline{T_{0}}$

A. $H(x)=\iota$ by the assumptions.

B. $(H ; E[\text { consume } x])^{e}=(H)^{e} ; E^{\prime}[$ consume $x$ ] where $E^{\prime}[\bullet]=\left(E^{\prime}\right)^{e}[\bullet]$ by Lemma B.26 with $t=E[$ consume $x]$.

C. $\left(H ; E[\text { consume } x] \overline{T_{0}}\right)^{e} \rightsquigarrow\left(H, x \mapsto \mathrm{T} ; E[v] \overline{T_{0}}\right)^{e}$ by application C-Eval, R-Consume with Item 2(a)iiiB, as required.

iv. R-FIELD. $H ; E[x . f]^{i} \overline{T_{0}} \rightsquigarrow H ; E[v]^{i} \overline{T_{0}}$

A. $H(x)=\iota, f=v \in \bar{F}, \neg$ islso $(H, v)$, localOwner $(H, i, \iota)$ by the assumptions.

B. $(H ; E[x . f])^{e}=(H)^{e} ; E^{\prime}[x . f]$ where $E^{\prime}[\bullet]=$ $\left(E^{\prime}\right)^{e}[\bullet]$ by Lemma B.26 with $t=E[x . f]$.

C. $(H(x))^{e}=\iota, f=v \in \bar{F}$, ᄀislso $\left((H)^{e},(v)^{e}\right)$, localOwner $\left((H)^{e}, i, \iota\right.$ by Definition B.11 with Item 2(a)ivA and Item 2(a)ivB. 
D. $\left(H ; E[x . f]^{i} \overline{T_{0}}\right)^{e} \leadsto\left(H ; E[v]^{i} \overline{T_{0}}\right)^{e}$ by application C-Eval, R-Field with Item 2(a)ivC, as required.

v. R-FieldAssign. $H ; E[x . f=v]^{i} \overline{T_{0}} \leadsto H^{\prime} ; E\left[v^{\prime}\right]^{i} \overline{T_{0}}$

A. $H(x)=\iota, f=v^{\prime} \in \bar{F}, \neg$ is $\operatorname{Imm}(H, \iota)$, $\operatorname{OkRef}(H, K, v)$, isLocal $(H, l) \Rightarrow($ isOwner $(H, i, l) \wedge$ localOwner $(H, i, v))$ by the assumptions.

B. $(H ; E[x . f=v])^{e}=(H)^{e} ; E^{\prime}[x . f=v]$ where $E^{\prime}[\bullet]=\left(E^{\prime}\right)^{e}[\bullet]$ by Lemma B.26 with $t=E[x . f=$ v].

C. $(H(x))^{e}=\iota, f=v^{\prime} \in \bar{F}, \neg$ is $\operatorname{Imm}(H, \iota)$, OkRef $(H$, unsafe, $v)$ by Definition B.11 with Items $2(a) v A$ and $2(\mathrm{a}) \mathrm{vB}$.

D. $\left(H ; E[x . f=v]^{i} \overline{T_{0}}\right)^{e} \leadsto\left(H^{\prime} ; E\left[v^{\prime}\right]^{i} \overline{T_{0}}\right)^{e}$ by application C-Eval, R-FieldAssign with Item 2(a)vC, as required.

vi. R-New. $H ; E[K \text { obj }\{\overline{f=v} \bar{M}\}]^{i} \overline{T_{0}} \rightsquigarrow H, \iota \mapsto K^{i}$ obj $\{\overline{f=v} \bar{M}\} ; E\left[v^{\prime}\right]^{i} \overline{T_{0}} \quad(H ; t \bar{T})^{e} \rightsquigarrow H^{\prime \prime} ; E r r$

A. $\forall f=v \in \overline{f=v} . \operatorname{OkRef}(H, K, v) \wedge(K=$ local $\wedge$ isLocal $(H, v)) \Rightarrow$ isOwner $(H, i, v), \iota$ fresh by the assumptions.

B. $(H ; E[K \text { obj }\{\overline{f=v} \bar{M}\}])^{e}=$ $(H)^{e} ; E^{\prime}[$ unsafe obj $\{\overline{f=v} \bar{M}\}]$ where $E^{\prime}[\bullet]=$ $\left(E^{\prime}\right)^{e}[\bullet]$ by Lemma B.26 with $t=E[K$ obj $\{\overline{f=v} \bar{M}\}]$.

C. $\forall f=v \in \overline{f=v}$. OkRef(H, unsafe, $v)$ by application of Definition B.11 with Items 2(a)viA and 2(a)viB.

D. $\left(H ; E[\text { unsafe obj }\{\overline{f=v} \bar{M}\}]^{i} \overline{T_{0}}\right)^{e} \leadsto H, \iota \mapsto$ unsafe ${ }^{i}$ obj $\{\overline{f=v} \bar{M}\} ; E\left[v^{\prime}\right]^{i}{\overline{T_{0}}}^{e}$ by application C-Eval, R-New with Item 2(a)viC, as required.

vii. R-CALL. $H ; E[x . m(v)] \overline{T_{0}} \leadsto H, x^{\prime} \mapsto \iota, y^{\prime} \mapsto$ $v ; E\left[t\left[\operatorname{sel} f=x^{\prime}\right]\left[y=y^{\prime}\right]\right]^{i} \overline{T_{0}}$

A. $x \mapsto \iota, \iota \mapsto \_$obj $\left\{\_\bar{M}\right.$ method $\left.m(y)\{t\}\right\}$ by the assumptions.

B. $(H ; E[x \cdot m(v)])^{e}=(H)^{e} ; E^{\prime}[x \cdot m(v)]$ where $E^{\prime}[\bullet]=$ $\left(E^{\prime}\right)^{e}[\bullet]$ by Lemma B.26 with $t=E[x . m(v)]$.

C. $x^{\prime}, y^{\prime}$ fresh by assumption $(\Gamma)^{e} \vdash\left(H ; E[x . m(v))^{e}\right]$

D. $\left(H ; E[x . m(v)] \overline{T_{0}}\right)^{e} \leadsto(H)^{e}, x^{\prime} \mapsto \iota, y^{\prime} \mapsto v ;(E)^{e}[(t[$ self $=$ $\left.\left.\left.x^{\prime}\right]\left[y=y^{\prime}\right]\right)^{e}\right] \bar{T}_{0}^{e}$ by application C-EvaL, RCALL with Items 2(a)viiA to 2(a)viiC, as required. viii. R-CAstLoc. $H ; E[(K) \iota] \overline{T_{0}} \rightsquigarrow H ; E[\iota] \overline{T_{0}}$

A. $\iota \in \operatorname{dom}(H)$ by the assumptions.

B. $(H ; E[(K) \iota])^{e}=(H)^{e} ; E^{\prime}[($ unsafe $) l]$ where $E^{\prime}[\bullet]=$ $\left(E^{\prime}\right)^{e}[\bullet]$ by Lemma B.26 with $t=E[(K) l]$.

C. $\iota \in \operatorname{dom}\left((H)^{e}\right)$ by assumption $(\Gamma)^{e} \vdash(H)^{e} ; E[(K) \iota]$

D. $\left(H ; E[(K) \iota] \overline{T_{0}}\right)^{e} \rightsquigarrow(H)^{e} ;(E)^{e}[\iota]\left(\overline{T_{0}}\right)^{e}$ by application C-Eval, R-CAsTLoc with Items 2(a)viiiA to 2(a)viiiC, as required.

ix. R-Copy. $H ; E[K \operatorname{copy} x]^{i} \overline{T_{0}} \rightsquigarrow H^{\prime} ; E[\iota] \overline{T_{0}}$
A. $x \in \operatorname{dom}(H)$ by the assumptions.

B. $(H ; E[K \text { copy } x])^{e}=(H)^{e} ; E^{\prime}$ [unsafe copy $\left.x\right]$ where $E^{\prime}[\bullet]=\left(E^{\prime}\right)^{e}[\bullet]$ by Lemma B.26 with $t=E[K \operatorname{copy} x]$.

C. $x \mapsto \iota^{\prime} \in(H)^{e}$ by assumption $(\Gamma)^{e} \vdash(H ; E[K \operatorname{copy} x])^{e}$

D. localOwner $\left(H, i, \iota^{\prime}\right)$ is vacuously satisfied.

E. $\left(H ; E[K \operatorname{copy} x] \overline{T_{0}}\right)^{e} \leadsto\left(H^{\prime \prime}\right)^{e} ;(E)^{e}[l]\left(\overline{T_{0}}\right)^{e}$ by application C-Eval, R-Copy with Items 2(a)ixA to 2(a)ixD, where $\left(H^{\prime}\right)^{e}=H^{\prime \prime}$, as required.

x. R-Spawn, R-Recv, R-SendBlock, and R-SendUnblock are all straightforward.

b. If $H ; t \overline{T_{0}} \rightsquigarrow H^{\prime} ; \overline{T_{1}} \overline{T_{0}}$ and $T_{-} 1=\operatorname{Err}_{A} \vee \operatorname{Err}_{N}$ then, $(H ; t \bar{T})^{e} \leadsto\left(H^{\prime \prime} ; \overline{T_{1}} \overline{T_{0}}\right)^{e}$

By induction on a derivation of $H ; t \overline{T_{0}} \rightsquigarrow H^{\prime} ; \overline{T_{1}} \overline{T_{0}}$ and by case analysis of $t$. Reminder: assumption $\overline{T_{1}}=\operatorname{Err}_{A} \vee \operatorname{Err}_{N}$. Need to show:

We proceed by induction on a derivation $H ; t \leadsto$ $H^{\prime} ; \overline{T_{1}}$.

- By all terms that use the derivation E-NoSuchField, E-NoSuchMethod, E-NoSuchFieldAssign, E-SendBadTarge? and E-RecvBADTARGET, a missing field or method in the unsafe configuration, $\left(H ; t \overline{T_{0}}\right)^{e} \rightsquigarrow H^{\prime \prime} ; \overline{T_{1}} \overline{T_{0}}$ reduces using the same reduction rule producing the same error.

- By all terms that use the derivation EAbsentVar, E-Consume, E-AbsentTarget, E-AbSENTTARgetAccess, E-AbSENTFIELdAssign, E-ABSEntCopyTARget, a missing target in the unsafe configuration, $(H ; t \bar{T})^{e} \rightsquigarrow H^{\prime \prime} ; E r r$ reduces using the same reduction rule producing the same error.

3. a. If $\left(H ; t \overline{T_{0}}\right)^{e} \leadsto\left(H^{\prime} ; E r r\right)^{e}$ then, $H ; t \overline{T_{0}} \leadsto H^{\prime} ; E r r$ By induction on a derivation of $\left(H ; t \overline{T_{0}}\right)^{e}$. Need to show:

$$
H ; t \overline{T_{0}} \rightsquigarrow H^{\prime} ; E r r
$$

E-NoSuchMethod, E-NoSuchFieldAssign, ESendBadTargetOrArgument, and ERecvBadTarget. Any configuration $\left(H ; t \overline{T_{0}}\right)^{e}$ that reduces to $\left(H^{\prime} ; E r r\right)^{e}$. By Lemma B.25 with $H ; t \quad \overline{T_{0}}$ deterministically takes a single reduction step. The fact that $H ; t \overline{T_{0}}$ may have safe capabilities does not stop normal errors to occur.

ii. E-AbsentVar, E-Consume, E-AbsentTarget, EAbsentTargetAccess, E-AbsentFieldAssign, EAbsentCopyTarget. From the induction hypothesis and the rules of the case, regardless of safe capabilities, the configuration will throw a $\operatorname{Err}_{A}$. 
b. If $\left(H ; t \overline{T_{0}}\right)^{e} \rightsquigarrow\left(H^{\prime} ; \bar{T} \overline{T_{0}}\right)^{e}$ and $\bar{T} \neq$ Errthen, $H ; t \overline{T_{0}} \leadsto$ $H^{\prime} ; \overline{T^{\prime}} \overline{T_{0}}$ and $\overline{T^{\prime}}=\operatorname{Err}_{P} \vee \operatorname{Err}_{C} \vee \bar{T}$.

By induction on a derivation of $\left(H ; t \overline{T_{0}}\right)^{e}$. Need to show:

$H ; t \overline{T_{0}} \rightsquigarrow H^{\prime} ; \overline{T^{\prime}} \overline{T_{0}} \wedge \overline{T^{\prime}}=\operatorname{Err}_{P} \vee \operatorname{Err}_{C} \vee \bar{T}$

i. R-LET. If $\left(H ; \text { let } x=v \text { in } t^{\prime} \overline{T_{0}}\right)^{e} \rightsquigarrow(H, x \mapsto$ $\left.v ; t^{\prime} \overline{T_{0}}\right)^{e}$, then $H ; t \overline{T_{0}} \leadsto H^{\prime} ; \overline{T^{\prime}} \overline{T_{0}}$ and $\overline{T^{\prime}}=\operatorname{Err}_{P} \vee$ $\operatorname{Err}_{C} \vee \bar{T} . x \notin \operatorname{dom}(H)$ by assumption $\Gamma \vdash H ; t \overline{T_{0}}$, and by application of R-LET, $H$; let $x=v$ in $t^{\prime} \overline{T_{0}} \leadsto$ $H, x \mapsto v ; t^{\prime} \overline{T_{0}}$ as required.

ii. R-VAR, $\left(H ; E[x] \overline{T_{0}}\right)^{e} \rightsquigarrow\left(H ; E[\iota] \overline{T_{0}}\right)^{e}$ under assumption $\neg$ islso $(H, \iota)$. We proceed by cases analysis over the capability of $\iota$ (omit the case were $H(x)=\mathrm{T}$, as that would have thrown an error in the hypothesis):

- $H(\iota)=$ iso obj $\{\ldots\}$. By application of E-AliAsIso, $H ; E[x] \overline{T_{0}} \rightsquigarrow\left(H ; \operatorname{Err}_{P} \overline{T_{0}}\right.$, as required.

- $H(\iota)=K$ obj $\left\{{ }_{--}\right\} \wedge K \neq$ iso. By application of R-VAR, $H ; E[x] \overline{T_{0}} \rightsquigarrow H ; E[\iota] \overline{T_{0}}$ as required.

iii. R-Consume, $\left(H ; E[\text { consume } x] \overline{T_{0}}\right)^{e} \rightsquigarrow(H[x \mapsto$ $\left.\mathrm{T}] ; E[\iota] \overline{T_{0}}\right)^{e}$. From the assumption of the derivation, $H(x)=\imath$. By application of R-Consume, $H ; E[$ consume $x] \overline{T_{0}} \leadsto H, x \mapsto \mathrm{T} ; E[\iota] \overline{T_{0}}$ as required.

iv. R-FIELD, $\left(H ; E[x . f]^{i} \overline{T_{0}}\right)^{e} \rightsquigarrow\left(H ; E[v]^{i} \overline{T_{0}}\right)^{e}$ under assumptions $H(x)=\iota, f=v \in \bar{F}$, ᄀislso $(H, v)$, and localOwner $(H, i, v)$. We proceed by case analysis over the capability of $\iota$.

- isLocal $(H, \iota)$. We proceed by case analysis over its ownership capability $K$ :

$-K^{i}$. We proceed by case analysis over $f$ :

* islso $(H, v)$. By E-IsoField, it reduces to $H$; $\operatorname{Err}_{P}$.

* ᄀislso $(H, v)$. By R-Field, $H ; E[x . f]^{i} \overline{T_{0}} \leadsto$ $H ; E[v]^{i} \bar{T}_{0}$ as required.

* $H(v)=K$ obj $\left\{{ }_{--}\right\} \wedge K \neq$ iso. By R-Field, $H ; E[x . f]^{i} \overline{T_{0}} \rightsquigarrow H ; E[v]^{i} \overline{T_{0}}$ as required.

- $K^{j} \wedge i \neq j$. By E-LocalField, it reduces to $H ; \operatorname{Err}_{P}$.

- islso $(H, v)$. By application of E-IsoField, the configuration steps to $H ; \operatorname{Err}_{P}$ as required.

- $\neg$ islso $(H, v)$. By cases analysis similar to the above case.

- Remaining cases. By R-Field, $H ; E[x . f]^{i} \overline{T_{0}} \leadsto$ $H ; E[v]^{i} \overline{T_{0}}$ as required.

v. R-FieldAssign, $\left(H ; E[x . f=v]^{i} \overline{T_{0}}\right)^{e} \leadsto\left(H^{\prime} ; E\left[v^{\prime}\right]^{i} \overline{T_{0}}\right)^{e}$. For all cases where $H(x)=\iota$ and $\neg \operatorname{OkRef}(H, K, \iota)$ by E-BADFIELDAssign, $H ; E[x . f=v]^{i} \overline{T_{0}} \leadsto H$; $\operatorname{Err}_{P}$ as required. For all cases where $\operatorname{OkRef}(H, K, \iota)$ we proceed by case analysis on the capability of $l$ :

- is $\operatorname{mm}(H, \iota)$. By E-BAdFieldAssign, $H ; E[x . f=$ v] $]^{i} \overline{T_{0}} \rightsquigarrow H ; \operatorname{Err}_{P}$ as required.

- $\neg \operatorname{is} \operatorname{Imm}(H, \iota)$
- notLocalOwner $(H, i, \iota)$. By E-BAdFieldAssign, $H ; E[x . f=$ $v]^{i} \overline{T_{0}} \rightsquigarrow H ; \operatorname{Err}_{P}$ as required.

- isLocal $(H, \iota) \wedge$ isOwner $(H, i, \iota)$. By case analysis on the capability of $f=v \in \bar{F}$.

- notLocalOwner $(H, i, v)$. By E-BAdFieldAssign, $H ; E[x . f=v]^{i} \overline{T_{0}} \rightsquigarrow H ; \operatorname{Err}_{P}$ as required.

- localOwner $(H, i, v)$. By R-FieldAssign, $H ; E[x . f=$ $v]^{i} \overline{T_{0}} \rightsquigarrow H^{\prime} ; E\left[v^{\prime}\right] \overline{T_{0}}$ as required.

- Remaining cases. By R-FieldAssign, $H ; E[x . f=$ $v]^{i} \overline{T_{0}} \rightsquigarrow H^{\prime} ; E\left[v^{\prime}\right] \overline{T_{0}}$ as required.

- Remaining cases. By R-FieldAssign, $H ; E[x . f=$ $v]^{i} \overline{T_{0}} \rightsquigarrow H^{\prime} ; E\left[v^{\prime}\right] \overline{T_{0}}$ as required.

vi. R-NEw, $\left(H ; E[K \text { obj }\{\overline{f=v} \bar{M}\}]^{i} \overline{T_{0}}\right)^{e} \leadsto\left(H^{\prime} ; E[l]^{i} \overline{T_{0}}\right)^{e}$.

For all cases where $\neg \operatorname{OkRef}(H, K, v) \forall f=v \in \overline{f=v}$, by application of E-BADINSTANTIATION, the configuration reduces to $H$; $\operatorname{Err}_{P}$. Under the assumption that $\operatorname{OkRef}(H, K, v) \forall f=v \in \overline{f=v}$ we proceed by case analysis over $K$ :

- $K=$ local. If $\exists f=v \in \overline{f=v}$, s.t. notLocalOwner $(H, i, v)$ then by direct application of E-BADINSTANTIATION the configuration reduces to $H$; $\operatorname{Err}_{P}$. Otherwise, by application of $R-N e w, H ; E[K \text { obj }\{\overline{f=v} \bar{M}\}]^{i} \overline{T_{0}} \leadsto$ $H^{\prime} ; E[l]^{i} \overline{T_{0}}$ as required.

- $K \neq$ local. Under the assumption $\operatorname{OkRef}(H, K, v) \forall f=$ $v \in \overline{f=v}$, by application of $R-N e w, H ; E[K \text { obj }\{\overline{f=v} \bar{M}\}]^{i} \bar{T}$ $H^{\prime} ; E[l]^{i} \overline{T_{0}}$ as required.

vii. The remaining rules follow the same pattern and structure.

Theorem B.28 (Multistep Dynamic Gradual Guarantee). A program $P$ reduces to a terminal configuration $C$ and generates a trace $\mathcal{T}$ from $\epsilon ; P \rightsquigarrow{ }^{*} H ; C$. C may or may not be an error configuration. Let $P^{\prime}$ be $P^{e}$, and let $n=|\mathcal{T}|$ :

1. If $\operatorname{Err}_{C} \neq C \neq E r r_{P}$, then $\mathcal{T} ; \epsilon ; P^{\prime} \rightsquigarrow{ }^{n} \mathcal{T}^{\prime} ; H ; C$

2. If $C \equiv \operatorname{Err}_{C} \vee \operatorname{Err}_{P}$, then $\mathcal{T} ; \epsilon ; P^{\prime} \rightsquigarrow{ }^{n} \mathcal{T}^{\prime} ; H^{\prime} ; \bar{T}$, and $\bar{T} \neq C$

- A program $P^{e}$ reduces to a terminal configuration $C$ and generates a trace $\mathcal{T}$ from $\epsilon ; P^{e} \rightsquigarrow^{*} H^{e} ; C$. C may or may not be an error configuration. Let $P^{\prime}$ be $P$, and let $n=|\mathcal{T}|$. If $P^{\prime}$ executes $\mathcal{T}$, then either:

1. if $C \not \equiv$ Err, then $\mathcal{T} ; \epsilon ; P^{\prime} \rightsquigarrow^{m} \mathcal{T}^{\prime} ; H^{\prime} ; C^{\prime}$ and $C^{\prime} \equiv$ $C \vee E r r_{P} \vee \operatorname{Err}_{C}$ and $m \leq n$.

2. if $C \equiv$ Err, then $\mathcal{T} ; \epsilon ; P^{\prime} \rightsquigarrow \rightsquigarrow^{n} \mathcal{T}^{\prime} ; H ; C$

The proof follows directly from Theorem 5.6. 